\title{
DEPLOYMENT OF ROBOT ARMS WITH A LEADER-FOLLOWER APPROACH FOR BOMB DISPOSAL
}

\author{
by \\ Trung Chau \\ B.Sc., Computer Science, Ryerson University 2008
}

\author{
A thesis \\ presented to Ryerson University \\ in partial fulfilment of the \\ requirements for the degree of \\ Master of Science \\ in the program of \\ Computer Science
}

Toronto, Ontario, Canada, 2020

(C) Trung Chau 2020 


\section{Author's Declaration for Electronic Submission of a Thesis}

I hereby declare that I am the sole author of this thesis. This is a true copy of the thesis, including any required final revisions, as accepted by my examiners.

I authorize Ryerson University to lend this thesis to other institutions or individuals for the purpose of scholarly research.

I further authorize Ryerson University to reproduce this thesis by photocopying or by other means,

in total or in part, at the request of other institutions or individuals for the purpose of scholarly research.

I understand that my thesis may be made electronically available to the public. 


\title{
DEPLOYMENT OF ROBOT ARMS WITH A LEADER-FOLLOWER APPROACH FOR BOMB DISPOSAL
}

\author{
Trung Chau \\ Master of Science, Computer Science, Ryerson University 2020
}

\begin{abstract}
Improvised Explosive Devices (IEDs) have been developed over the years across many nations around the world. IEDs used by terrorist actions and in warfare cause devastating death, injuries and damage. To protect the public, many emergency responders have to risk their lives by performing extremely hazardous tasks such as interacting with suspected IEDs. To prevent the emergency response teams from being negatively impacted by IEDs, many different kinds of response robots have been deployed in many locations worldwide - allowing first responders a safe way to interact with these menaces from a distance. This thesis contributes to the understanding of using robot arms with a Leader-Follower (LF) approach to help humans with performing dexterous operations like those which are inevitably required for manipulating IEDs remotely. The LF approach allows operators to remotely manipulate a robot arm without putting operators' lives in danger. By physically controlling one arm from a safe distance, operators can successfully copy its movements to a second arm. As a result, we argue, this approach can be helpful for minimizing operator risk when interacting with suspicious devices while at the same time facilitating more intuitive remote control.
\end{abstract}




\section{Acknowledgements}

I would like to express my sincere gratitude to my thesis supervisor, Dr. Alex Ferworn for all his support, motivation, enthusiasm, and patience. Under his guidance, I was able to extend my knowledge in developing technologies that could be used to contribute to future research.

I would like to acknowledge the support that I have received from my co-supervisor, Dr. Isaac Woungang, and everyone in the Network-Centric Applied Research Team (N-CART) research lab.

I am extremely grateful to Dr. Jimmy Tran and Dr. James Elliott Coleshill for their great support, experience and invaluable advice.

Many thanks to Dr. Jason Nolan for providing the use of his equipment, Kinova robot arms, and Martin Ristov for his technical support. I have garnered a lot of technical knowledge from these great individuals.

The person that I would like to thank the most is my Mother who has supported and encouraged me to pursue this thesis research.

Last, special thanks are owed to the Ryerson University Library Collaboratory, for the use of their facilities and equipment to conduct evaluation exercises and all operators who devoted their spare time to sharing their testing experiences to support this thesis research. Without their help, my work would have not been possible. 


\section{Table of Contents}

Abstract..........................................................................

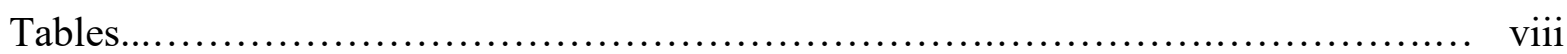

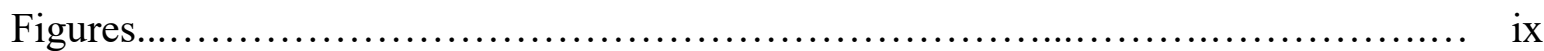

Equations................................................................ xi

Appendices............................................................. xii

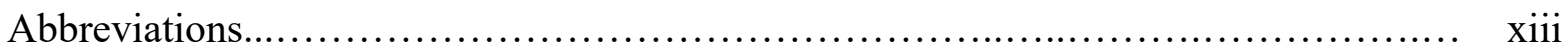

Chapter 1: Introduction

1.1 The Evolution of Robot Technology .................................... 1

1.2 Improvised Explosive Devices...................................... 3

1.3 The Problem...................................................... 4

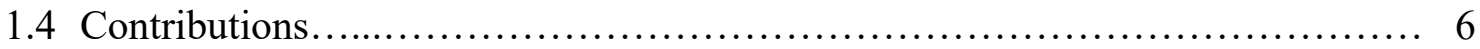

1.5 Thesis Organization.................................................. 7

Chapter 2: Literature Review

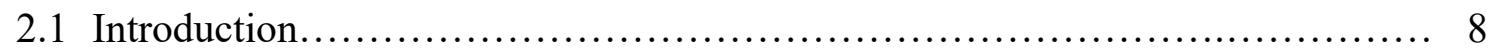

2.2 Important Capabilities of Response Robots.............................. 8

2.2.1 Ground Response Robots....................................... 9

2.2.2 Other Types of Response Robots................................. 11

2.3 Advanced Teleoperation Systems........................................ 13

2.3.1 Leap Motion Controller......................................... 13

2.3.2 Real-time Glove Control......................................... 15 
2.3.3 Virtual Joystick...................................................... 17

2.3.4 Master-Slave Control System........................................... 18

2.4 Fundamental Concepts of Robot Manipulators................................ 19

2.4.1 Forward Kinematics...................................................... 19

2.4.2 Inverse Kinematics................................................... 22

2.4.3 Degree(s) of Freedom................................................ 22

2.5 Standardized Test Methods.................................................... 23

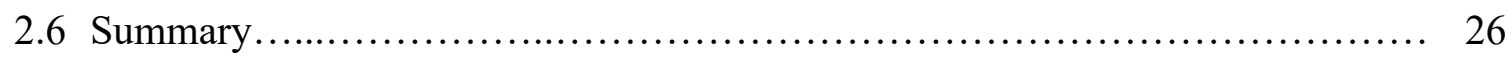

Chapter 3: Technical Approach

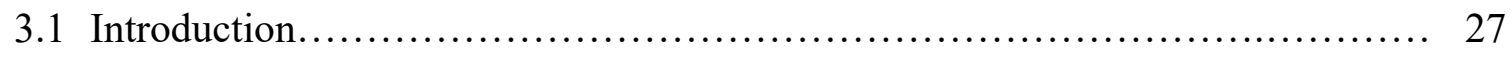

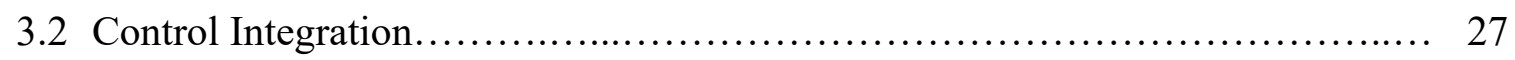

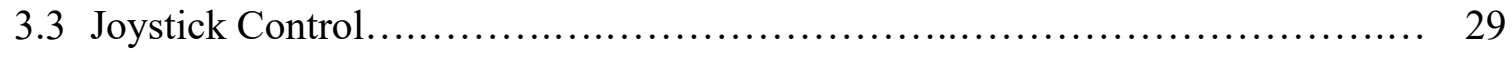

3.4 Leader-Follower Approach................................................ 34

3.5 Test Methodologies............................................................ 35

3.5.1 Standard Metrics.................................................... 36

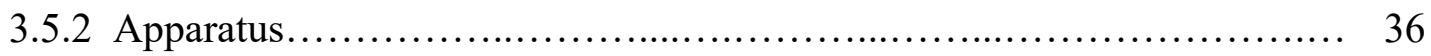

3.5.3 Testing Procedures..................................................... 43

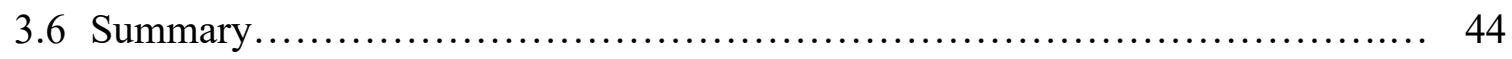

Chapter 4: Experiments and Results

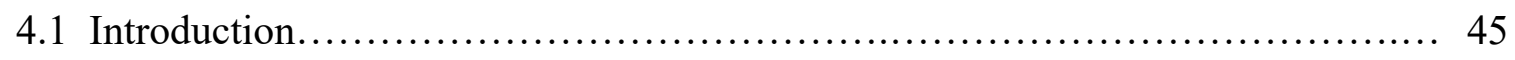

4.2 Experimental Results...................................................... 46 
4.2.1 Experimental Results for Controlling in Angular Mode................. 46

4.2.2 Experimental Results for Controlling in 3-Axis Mode................. 50

4.2.3 Experimental Results for the LF Method........................... 54

4.3 The Challenge....................................................... 57

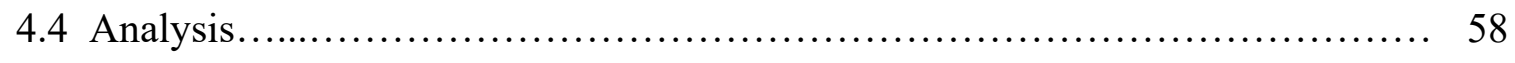

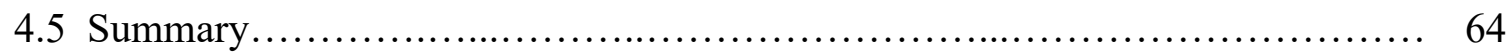

Chapter 5: Conclusion and Future Work

5.1 Conclusion....................................................... 65

5.2 Future Work..................................................... 67

Bibiliography............................................................ 74 


\section{List of Tables}

Table 2.4.1: $\quad$ Four Denavit-Hartenberg parameters.............................. 21

Table 3.3: $\quad$ 2-Axis operation mode...................................... 32

Table 3.5.3: Time measured in seconds when using various test modes............... 43

Table 4.2.1: $\quad$ Overall average and total time measured in seconds in Angular mode.... 48

Table 4.2.2: $\quad$ Overall average and total time measured in seconds in 3-Axis mode...... 52

Table 4.2.3: $\quad$ Overall average and total time measured in seconds in the LF mode..... 55

Table 4.4: $\quad$ Data comparison of the mean and the STD $\ldots \ldots \ldots \ldots \ldots \ldots \ldots \ldots \ldots \ldots . \ldots 2$ 


\section{List of Figures}

Figure 1.1: $\quad$ Technical specifications of Universal Robot......................... 2

Figure 1.2: Chelsea explosion, a bomb blast in NewYork City.................... 3

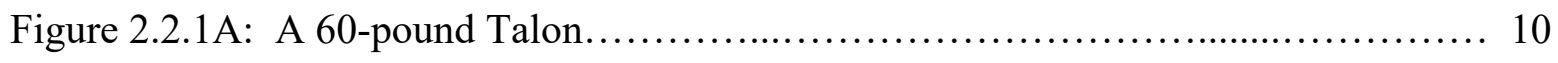

Figure 2.2.1B: The Vanguard MK II-T $\ldots \ldots \ldots \ldots \ldots \ldots \ldots \ldots \ldots \ldots \ldots \ldots \ldots \ldots \ldots \ldots \ldots$

Figure 2.2.2A: A Mavic 2 Pro................................................ 11

Figure 2.2.2B: First Underwater ROV used in 1960's.......................... 12

Figure 2.3.1A: A typical Leap Motion Controller interface........................... 13

Figure 2.3.1B: Overall information flow diagram when using the LMC .............. 14

Figure 2.3.1C: Grasp and release operations................................. 15

Figure 2.3.2: Glove controller with flex sensors, IMU and Arduino................. 16

Figure 2.3.3: Virtual joystick (GUI) ..................................... 17

Figure 2.3.4: A typical master-slave control system........................... 18

Figure 2.4.1: Coordinate frame assignment for a general manipulator................. 20

Figure 2.4.3: The six degrees of freedom of movement of a ship.................... 22

Figure 2.5: $\quad$ Examples of test methods conducted by NIST ........................ 24

Figure 3.3.1: Kinova $\mathrm{MICO}^{2}$ arm in HOME and RETRACTED position.............. 29

Figure 3.3.2: 3 -Axis operation mode..................................... 31

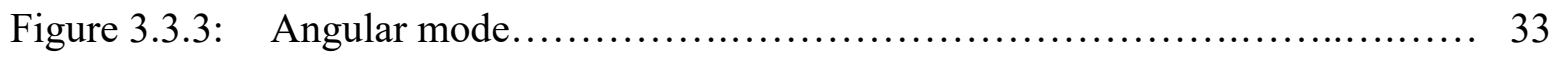

Figure 3.5.2A: Mounting plate used for inserting a robot arm on top................ 37

Figure 3.5.2B: Base of the $\mathrm{MICO}^{2}$ arm before being mounted.................... 37 
Figure 3.5.2C: Kinova 6 DOF curved wrist $\mathrm{MICO}^{2}$ arm part ID.................... 38

Figure 3.5.2D: Gripper of $\mathrm{MICO}^{2}$ arm with three fingers......................... 39

Figure 3.5.2E: Lightweight objects used for grasping and dropping................. 40

Figure 3.5.2F: Custom divider used to separate two robot arms................... 40

Figure 3.5.2G: Bullet camera attached to the gripper wrist....................... 41

Figure 3.5.2H: Interconnection diagram illustrates the experimental setup.............. 42

Figure 4.2.1A: Kinova $\mathrm{MICO}^{2} \mathrm{arm}$ in several distinct poses....................... 47

Figure 4.2.1B: Average time measured in seconds per task in Angular mode............ 49

Figure 4.2.2A: Various poses transformed when in 3-Axis mode.................... 51

Figure 4.2.2B: Average time measured in seconds per task in 3-Axis mode.......... 53

Figure 4.2.3A: Robot arms synchronized in the LF mode...................... 54

Figure 4.2.3B: Average time measured in seconds per task in the LF mode............. 56

Figure 4.4.1: $\quad$ Simulated pipe bombs used during experiments..................... 59

Figure 4.4.2: $\quad$ Average time per task and average total time needed................ 60

Figure 4.4.3: Number of successful tests completed............................ 61 


\section{List of Equations}

Equation 1: Transformations............................................ 20

Equation 2: The forward kinematics of a six joint robot arm.................... 21

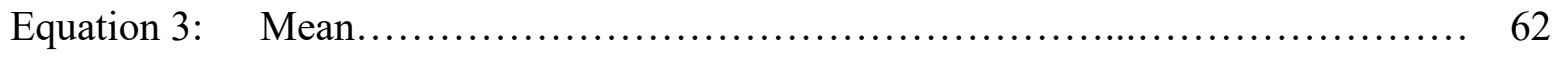

Equation 4: Standard Deviation............................................ 62 


\section{List of Appendices}

Appendix A: Feedback on Control Mode

Figure A: Kinova joystick with different statuses of blue LEDs feedback........... 69

Appendix B: Fixed Camera Views of the Apparatus

Figure B: A quad display providing views of the apparatus from 3 cameras........ 70

Appendix C: Software Limitations of Actuators and Fingers

Table $\mathrm{C} 1:$ Kinova $\mathrm{MICO}^{2}$ arm software limitations of actuators................ 71

Table C2: Kinova $\mathrm{MICO}^{2}$ arm software limitations of fingers................... 71

Appendix D: Singularity Auto-avoidance Behaviour

Figure D1: Kinova 6 DOF $\mathrm{MICO}^{2}$ arm with boundary singularity................... 72

Figure D2: Kinova 6 DOF MICO $^{2}$ arm with wrist-over-base singularity.............. 73 
Abbreviations

IED

LF

N-CART

EOD

EDU

WTC

NIST

VBIED

PBIED

SWORDS

HPDBS

DARPA

UAS

UROV

DOF

LMC

IMU

ROS

HMI

D-H

DHS S\&T

API

GUI

SDK

ASTM

CBRNE
Improvised Explosive Device

Leader-Follower

Network-Centric Applied Research Team

Explosive Ordnance Disposal

Explosive Disposal Units

World Trade Center

National Institute of Standards and Technology

Vehicle-borne IED

Person-borne IED

Special Weapons Observation Reconnaissance Detection System

Houston Police Department Bomb Squad

Defense Advanced Research Project Agency

Unmanned Aerial System

Underwater Remotely Operated Vehicle

Degree of Freedom

Leap Motion Controller

Inertial Measurement Unit

Robot Operating System

Human-Machine Interface

Denavit-Hartenberg

Department of Homeland Security, Science and Technology

Application Interface

Graphical User Interface

Software Development Kit

American Society for Testing and Materials

Chemical, Biological, Radiological, Nuclear and Explosive 


\section{Chapter 1}

\section{Introduction}

\subsection{The Evolution of Robot Technology}

According to [1], robotics is a branch of artificial intelligence which deals with the design, construction, operation, and application of robots. There are many different kinds of robots and each has been designed and used in various areas to assist humans with supporting a lot of disabled as well as elderly people in various tasks in their daily activities, investigating hazardous environments, and performing many risky tasks from safer distances, etc. As robots become more sophisticated, many of advanced robots [2-3] are capable of investigating and accessing to a burning building to search for people stuck inside, whereas others have the capabilities to detect hidden grenades or explosives [4]. These robots are extremely helpful in saving lives because they could be used to perform many extremely hazardous tasks even though they are not at a level to replace humans yet.

A robot arm is a type of mechanical arm, usually programmable, with similar functions to a human arm [5]. There are many different companies that make robot arms available to businesses of every size [6], all over the world. Most of them are designed to mimic the range of the motion of a human arm with different types of payloads and weights [7]. Most robot arms are usually light in weight and flexible designs [8-9]; therefore, they can be easily transported from one environment to another and/or mounted on tables or wheelchairs to assist humans with handling basic tasks such as picking up a bottle of water or moving small items around. 

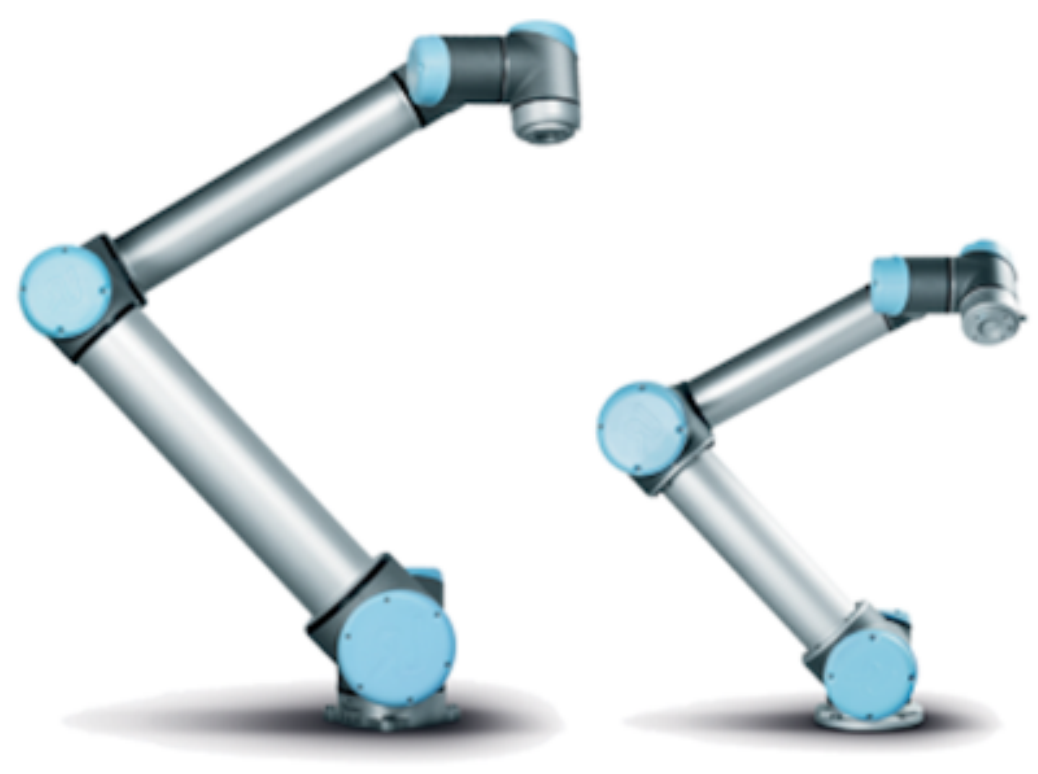

\begin{tabular}{|llll|}
\hline & UR10 robot arm & UR5 robot arm & UR3 robot arm \\
\hline Working radius & $1300 \mathrm{~mm} / 51.2 \mathrm{ins}$ & $850 \mathrm{~mm} / 33.5 \mathrm{ins}$ & $500 \mathrm{~mm} / 19.7$ ins \\
Payload & $10 \mathrm{~kg} / 22 \mathrm{lbs}$ & $5 \mathrm{~kg} / 11 \mathrm{lbs}$ & $3 \mathrm{~kg} / 6.6 \mathrm{lbs}$ \\
Weight & $28.9 \mathrm{~kg} / 63.7 \mathrm{lbs}$ & $18.4 \mathrm{~kg} / 40.6 \mathrm{lbs}$ & $11 \mathrm{~kg} / 24.3 \mathrm{lbs}$ \\
Footprint & $190 \mathrm{~mm} / 7.5$ ins & $149 \mathrm{~mm} / 5.9 \mathrm{ins}$ & $118 \mathrm{~mm} / 4.6 \mathrm{ins}$ \\
\hline
\end{tabular}

Figure 1.1 - Technical specifications of Universal Robot model UR3/ UR5/ UR10 [7]

More importantly, some high-tech robots can be used to save lives by operating many perilous tasks which are risky for humans to do. These robots are known as "Bomb Disposal Robots" or "Explosive Ordnance Disposal (EOD) robots" [10-13], which has been widely used in military services and many dangerous environments for emergency rescue support, bomb disposal, tactical operations, surveillance, etc. to inspect for suspicious packages and neutralize munitions or bombs if needed [14-16]. These advanced EOD robots have been developed over the years by researches with the objective of improving safety of the following operations: search and rescue missions, IED-related tasks, or bomb disposal in land operations, etc. 


\subsection{Improvised Explosive Devices (IEDs)}

An attack based on an Improvised Explosive Device, or IED [17] had threatened all New York City residents and their neighborhoods on the evening of Saturday, September 17. It was confirmed that the bomb exploded in the attack was a pressure-cooker device, which was similar to the one used in the Boston Marathon attack in 2013 [18]. These bombs are called IEDs and have been widely used by most of terrorists and insurgents to cause severe damage and induce panic in the public.

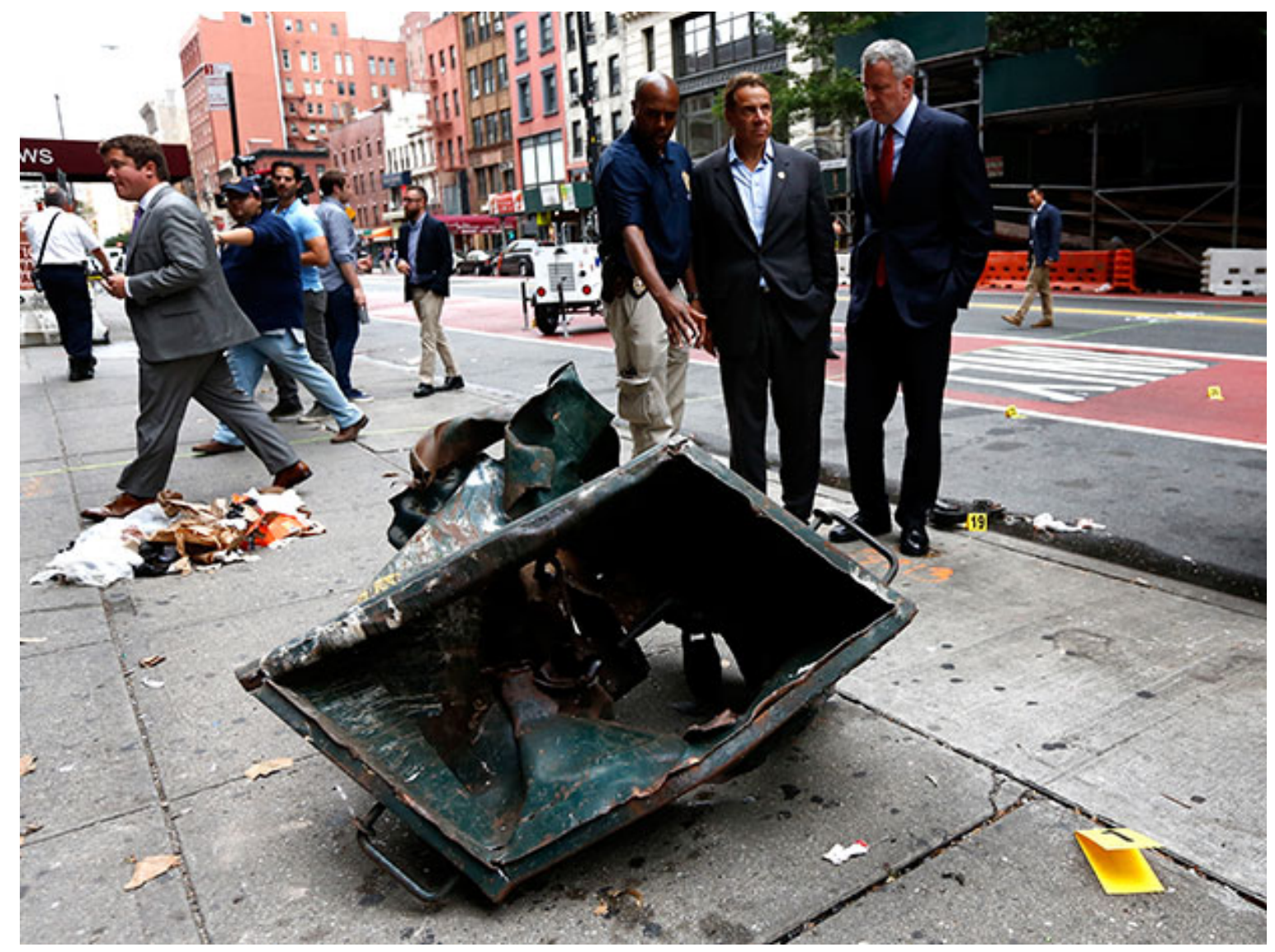

Figure 1.2 - Chelsea explosion, a bomb blast in NewYork City, which injured 29 people [17]

According to [19], during the Vietnam War, IEDs were used by the Viet Cong to fight against land- and river-borne vehicles; moreover, another large-scale use of IEDs derailed thousands of 
German trains [20] which caused a lot of damage or destruction during 1943-1944. With the growth of the development in science and technology, insurgents and terrorists worldwide can easily make IEDs the fastest growing type of weapon by simply using available bomb-making instructions on the Internet. As a result, more and more specialized response teams, called "Explosive Disposal Units" (EDU), have been urged to help with searching for the use of hidden explosives in urban environments. The challenge is to find a safe and effective way to neutralize the threat associated with explosive devices by either disabling or safely detonating them from a safe distance. For this reason, many advanced bomb disposal robots have been deployed to help emergency responders with performing hazardous tasks in order to save innumerable lives.

\subsection{The Problem}

Many studies [21-25] have been conducted since the attacks of Sept. 11, 2001 at the World Trade Center (WTC) Towers to reduce the risk to first responders. As a consequence, a series of security initiatives have also been undertaken with a primary consideration to protect first responders from being unforeseeably impacted by hidden IEDs. In fact, terrorist attacks continue to take place even more serious at open-access events such as marathons, parades, political rallies, protests, festivals, etc. Regardless of the types of events, as the threat of IEDs continues to grow, more and more bomb squads are in demand and should be better prepared to prevent, respond to, and mitigate the negative effects of terrorist attacks. Therefore, it is imperative to reconsider, and perhaps reduce the potential risks that might affect the safety of emergency response teams while performing their duties. Instead of sending human first responders to physically interact with items or other objects that might conceal IEDs, many advanced rescue robots have been extensively deployed to minimize risks while performing search and exploration tasks. 
One type of robot that can be helpful for providing a promising solution to assist emergency response teams in time-critical missions is the EOD robot. Typically, bomb disposal robots [2629] are equipped with cameras and a two-way communication link that allows them to be teleoperated from safe standoff distances with the intention of dealing with explosive devices by first searching and recognizing, then moving, lifting and/or safely disposing unknown suspected packages while keeping investigators out of harm's way. Although various techniques have been proposed and used to deploy these bomb robots, all of which take time for operators to first familiarize themselves with different situations prior to applying control techniques requiring remote operations in dangerous environments whereas others might fail to render the IED effectively in a timely manner. These bomb robots require some special training in addition to special knowledge about robot control. The learning process is laborious and time consuming. Different scenarios may take longer time for operators to plan out a route so that robots can efficiently navigate to target destinations, especially when the environments are complex. Up until now these bomb robots have been used in well-controlled manipulations by police officers, bomb squads, and/or emergency responders among various situations and complex environments with variable terrains, many assorted obstacles, confined areas, etc. With the environments and situations given, there has been many advanced solutions to help operators with remotely controlling bomb robots without risking their lives. Nevertheless, what is the most flexible, reliable, and intuitive way for operators to effectively maneuver such a remote-controlled robot when dealing with suspicious items while at the same time minimizing the loss or injury to operators? 


\subsection{Contributions}

This thesis makes the following contributions:

1. A new method for robotic arm control for reducing the risk to emergency response teams.

2. The first to introduce side-by-side comparisons of different test methods adapted from a standard set of test methods being developed by the National Institute of Standards and Technology (NIST) with the main goal of improving safety related manipulation tasks.

3. Provides experimental evidence that the Leader-Follower approach for remote manipulation is superior to common control mechanism currently used for EOD robot operations.

The first contribution of this thesis is the proposal of a new method which allows operators to use one local arm to manipulate the movement of another remote arm at a distance, called the LeaderFollower approach. Some of these tasks could be simple but are too dangerous for humans to perform whereas other tasks are not only complex but also time consuming. In terms of safety related manipulation tasks, this new control strategy have been developed to help first responders with performing various hazardous tasks while at the same time minimizing their risk when interacting with suspected IEDs.

The second contribution introduces the development of collecting capability data concerning response robots and facilitating direct comparisons among different control strategies when deploying robot arms for remote manipulation tasks. As a consequence, reported findings and data comparisons can be used to identify the efficacy of the Leader-Follower approach in relation to other control strategies when determining the most effective way of remotely controlling a robot arm using identical tasks allowing "apples to apples" comparisons. 
Lastly, the Leader-Follower method introduced in this thesis is not only simple to implement but also flexible and intuitive to use when performing various complicated movements as compared with a traditional way using a joystick. This integrated system is developed to support robot operators with various applications requiring remote operation with a robot arm. Regardless of which application considering during the deployment process, we believe the LF method is the most advantageous control technique for various levels of robot operators and thus, overcomes other test methods in general.

\subsection{Thesis Organization}

This thesis is organized as follows:

Chapter 2 presents background information related to this thesis, including a literature review of important capabilities of response robots, high-level teleoperation systems, fundamental concepts of robot manipulators and an introduction to standardized test methods.

Chapter 3 describes the details of general control principles and an overview of the testing environment including test equipment, procedures and how we apply the Leader-Follower approach to a remote robotic manipulation system.

Chapter 4 discusses experimental results, operational challenges and analysis of data collected during experiments defined in Chapter 3.

Chapter 5 provides final conclusions, discusses limitations and potential future work. 


\section{Chapter 2}

\section{Literature Review}

\subsection{Introduction}

While it may be impossible to eliminate the threat of an IED attack, a challenge for developing safe and effective ways to remotely interact with hidden explosives might help first responders to minimize their risk. This chapter presents a review of the importance of different kinds of response robots' capabilities found in various equipment. In addition, we also discuss various schemesboth operational and experimental-for teleoperation systems as well as several fundamental concepts of robot manipulators. Last but more importantly, the chapter goes on to describe various standard test methods conducted by NIST.

\subsection{Important Capabilities of Response Robots}

Most insurgents and terrorists construct IEDs in various sizes and in an increasing number of configurations including vehicle-borne IEDs (VBIEDs) [30-32], such as a truck bomb and personborne IEDs (PBIEDs) [33], such as a backpack bomb. Several famous attacks using IEDs occurred against the World Trade Center in New York City in 1993 [34][35] and the Murrah Federal Building in Oklahoma City in 1995 [36-38]. Each attack caused devastating damage and injuries to people and neighbourhoods. As these types of IEDs continue posing significant dangers to the public, and potentially emergency responders, various human-like components of robots have been developed and slowly optimized to improve performance to meet the needs of safety-critical incidents. Various situations require different combinations of robotic technologies to be deployed 
with different capabilities in response to various mission profiles. The following subsections describe the significant capabilities of different types of response robots available.

\subsubsection{Ground Response Robots}

The very first bomb disposal robot was designed to place a hook under a car suspected of containing a bomb in order to tow the car to a safe location without putting an EOD officer in danger. This initial Wheelbarrow EOD robot was invented in 1972 by Lieutenant-Colonel Peter Miller [39][40]. Many other types of robot technology have evolved in bomb-response operations [41-47]. Robots have emerged with widely different capabilities and come equipped with pincers, disruptors, and jammers capable of dealing with many types of IEDs. According to estimates from [48], about $60 \%$ of all explosive devices were safely neutralized in Northern Ireland where a total of 400 EODs were destroyed or damaged in operation, and this saved many lives. Ground response robots can also be used to perform various common tasks such as removing an object from an entrance, opening doors, climbing stairs, and using tools to carry out many other dexterous tasks [49-51]. According to [52], the first three armed Talon SWORDS (Special Weapons Observation

Reconnaissance Detection System) robots were sent to Iraq in June 2007 by the US Army. The teleoperated "Talon" robot, as illustrated in Figure 2.2.1A below, are popular unmanned vehicles used in bomb disposal operations by the US and several other countries. This particular robot weighs about 60 pounds and can travel at 5.2 miles per hour a payload of $200 \mathrm{lb}$. Another example of a small bomb disposal robot [53] was selected by the Houston Police Department Bomb Squad (HPDBS) in order to evaluate its performance, called Vanguard MKII-T. The robot has a wide range of capabilities to deal with many IED-related common tasks (Figure 2.2.1B). 


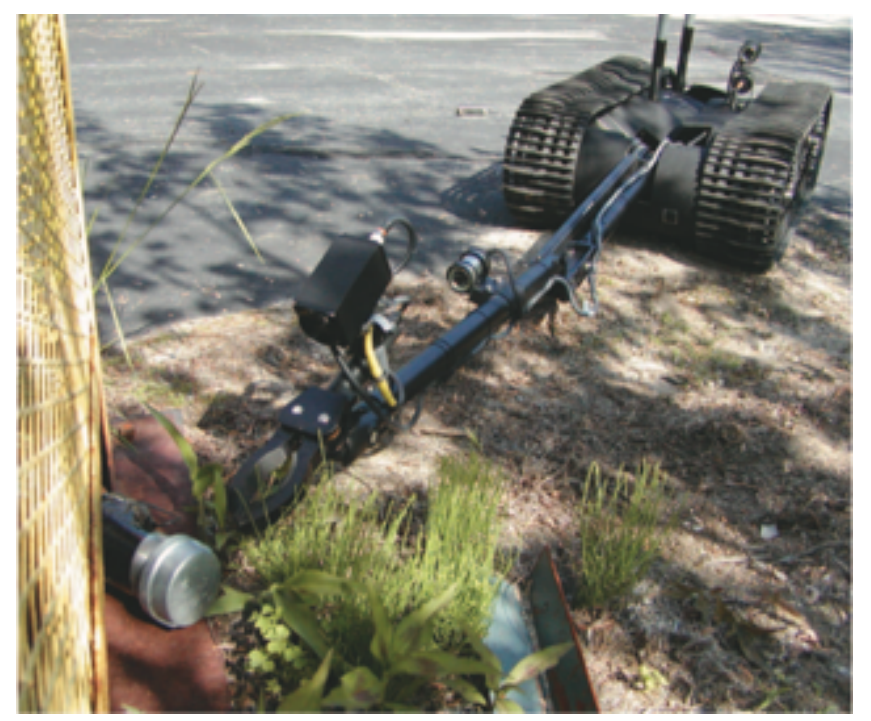

Figure 2.2.1A - A $60 \mathrm{lb}$ Talon can handle up to a $200 \mathrm{lb}$ payload [51]

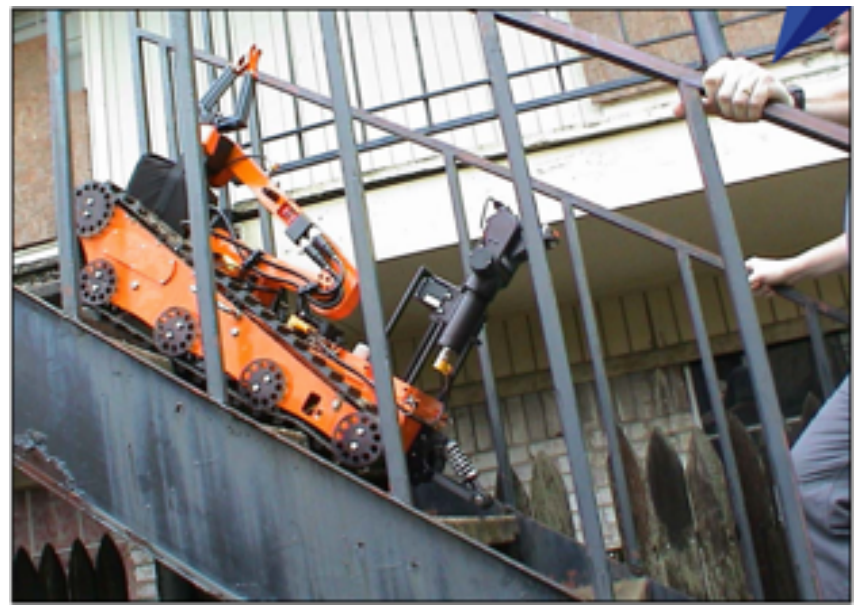

Figure 2.2.1B - The Vanguard MK II-T is capable of climbing up an ascend stair [53]

According to the Defense Advanced Research Project Agency [54-58], or DARPA, many response robots are very helpful for performing various hazardous tasks during or in the aftermath of a disaster. The goal of search and rescue operations is to find and rescue the largest number of victims in the shortest time possible, while minimizing potential risks to rescuers. It is impossible for one single rescue robot to deal with all different types of obstacles, challenges and tasks of an 
environment. As a result, cooperation between emergency response teams and robots as well as coordination among multi-robots [59-62] is extremely important for successfully completing rescue missions.

\subsubsection{Other Types of Response Robots}

To improve efficiency in search and rescue tasks, aerial response robots--unmanned aerial systems (UASs), have been widely deployed in various situations, such as large search area or environment extremely difficult to access by ground response robots [63]. For example, they can be used to track the spread of wildfires, search for missing people and detect toxic fumes after an explosion prior to sending human first responders [64-66]. More importantly, these drones are very helpful for navigating around hallways inside buildings, searching and mapping wide areas, and avoiding some unanticipated situations that may interfere with radio communications [67-70]. While operating drones in circular orbits or spiral movements, emergency response drone pilots are able to identify hidden objects from a far-off distance and subsequently, perform many other tasks, such as reading a hazardous material label or searching for essential mission tasks [71].

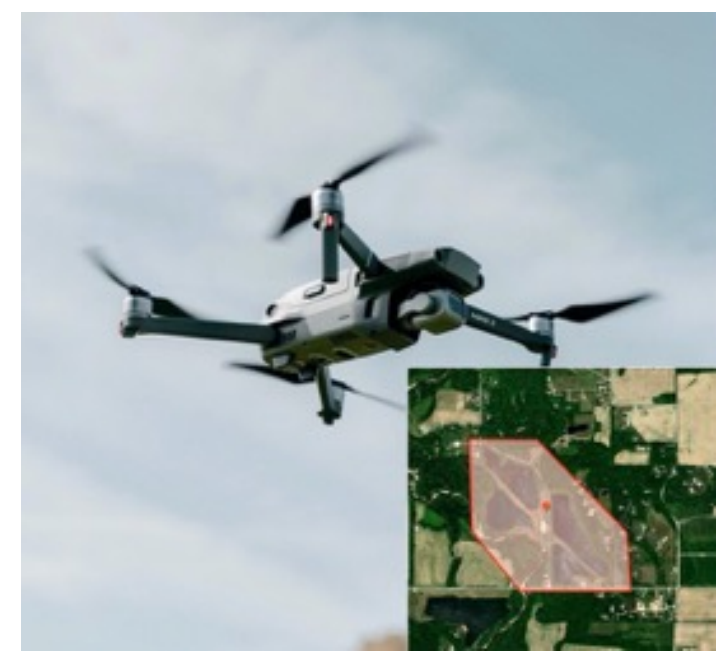

Figure 2.2.2A - A Mavic 2 Pro used in search and rescue operations [72] 
With the use of the Mavic 2 Pro as shown in Figure 2.2.2A above, first responders are able to remotely locate specific targets or a missing individual in large areas such as an open field, a forest or a lake, etc. while at the same time cutting down search times significantly compared to a traditional on-foot search and rescue option [72-74].

Another type of response robot, called "underwater remotely operated vehicle" (UROV), is a submersible and capable of cooperating with ground and air based robots to help emergency responders with numerous military applications [75-76]. With the support of UROVs, without directly interacting with suspicious floating objects, emergency responders are able to grasp and drag them away to other designated locations on the water surface. The use of underwater response robots may also reduce the likelihood of further injuries for emergency response teams [77][78] while at the same time improving efficiency in search and exploration tasks in the marine environments where fishing nets and wildlife might pose dangers.

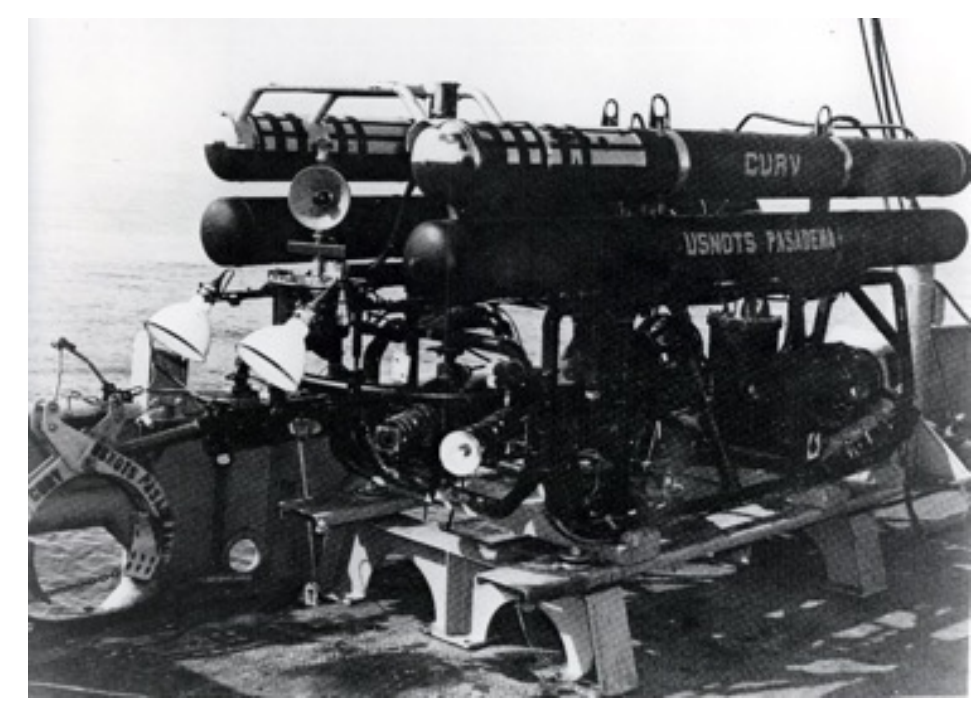

Figure 2.2.2B - First Underwater ROV used in the 1960's by the U.S. Navy [79]

Figure 2.2.2B is a remotely operated vehicle first used by the U.S. Navy to successfully search for an atomic bomb that went missing off the coast of Spain in the 1960's. 


\subsection{Advanced Teleoperation Systems}

Teleoperation is the primary method used in robotic control even though many forms of autonomous control have been proposed [80-81]. A teleoperated robot is a programmed mechanical device which can be controlled by a remote operator using a computer or other interface from a safe distance to either inspect, search, or access distant and often dangerous areas [82-84]. Teleoperated system or telemanipulation allows operators to remotely interact with other objects in dangerous and/or unreachable environments such as those with toxic gas, fires, and radiation [85].

\subsubsection{Leap Motion Controller}

One of the most common ways to control a robot is to use a joystick controller. Depending on the complexity of robotic systems, some may involve more or less cognitive load for robots' operator. In order to facilitate a more natural method of interaction for controlling mobile robots or to make the operator's control task easier, research has been conducted on the performance improvement of advanced teleoperation systems, including the development of the Leap Motion technology [8691]. According to [92], Hayden was able to produce an intuitive and adaptive approach that successfully mimics the motion of a human hand by using a 6 Degree of Freedom (DOF) Leap Motion Controller (LMC), as shown in the figure below.

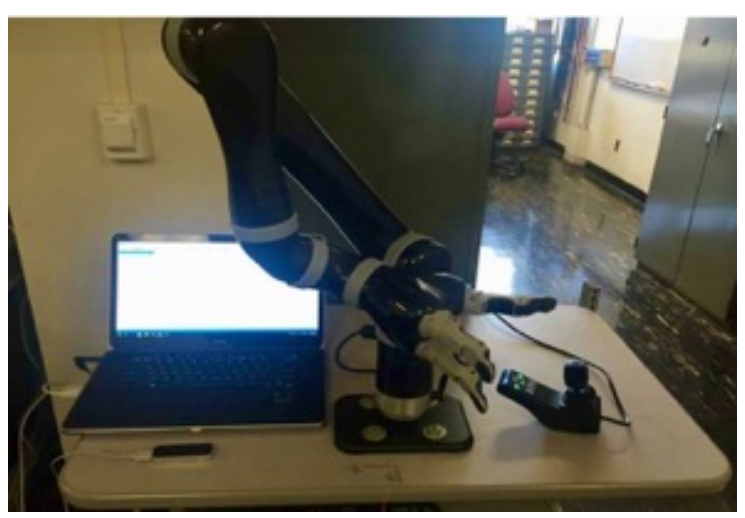

Figure 2.3.1A - A typical Leap Motion Controller interface [92] 
A 6-DOF JACO robotic arm, as shown in Figure 2.3.1A, which is made by Kinova, was selected in his research. By developing a communication interface between the Leap Motion controller and the robotic arm, the standard Kinova joystick, originally designed to operate a robotic arm, was replaced with the Leap Motion controller. Thus, there is no need to switch between different control modes such as translation, wrist, or fingers when controlling the JACO robotic arm.

The implementation of this work was done in the $\mathrm{C}++$ programming language in order to receive the data input from a Leap sensor in Cartesian coordinates. These values were scaled respectively before being translated into a new set of $\mathrm{x}, \mathrm{y}, \mathrm{z}$ coordinates required to move a robotic arm. This advanced technique provides a new novel gesture and position tracking system which is based on infrared optics and cameras instead of depth sensors. The following diagram (Figure 2.3.1B) describes in detail how all information regarding the user's current positions $(\mathrm{X}, \mathrm{Y}$, and $\mathrm{Z})$ can be retrieved from the Leap Motion controller and transferred into the Leap SDK for further mapping algorithm prior to being sent to the JACO robotic arm.

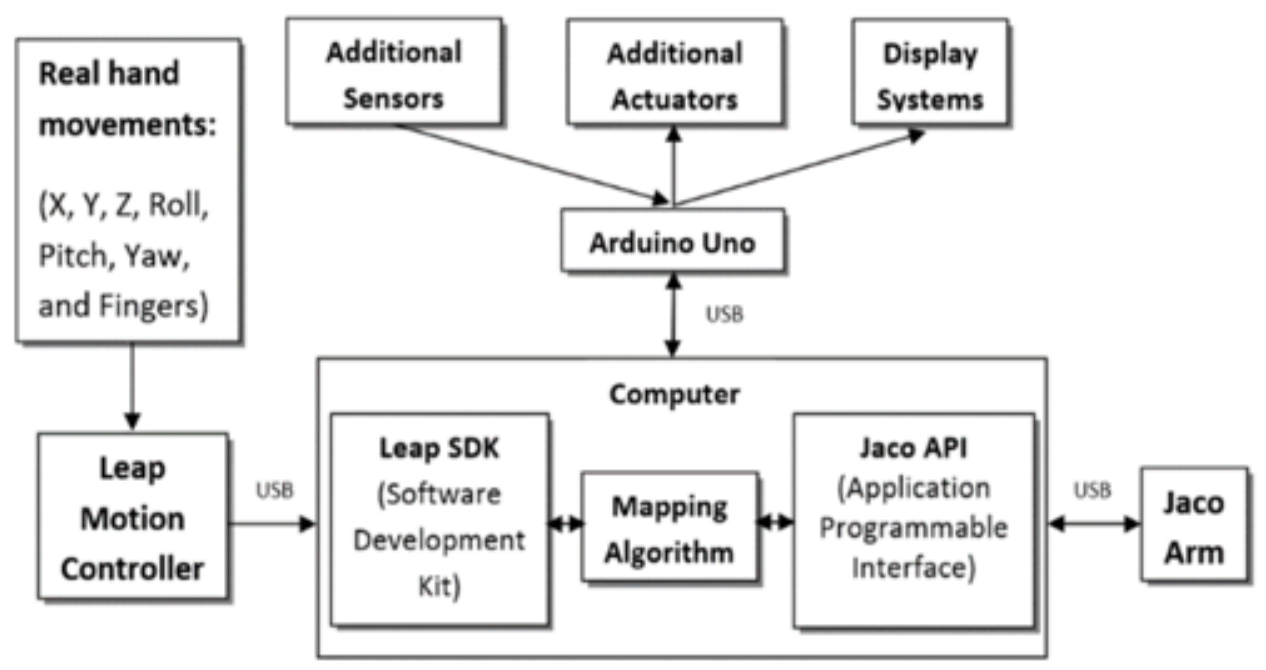

Figure 2.3.1B - Overall information flow diagram when using the LMC [93] 
In addition, there is an extra interface between the developed software and the Arduino Uno microcontroller required to enhance other features of the system such as additional sensors, actuators, and display components (LEDs, push buttons, etc.).

Overall, this approach was done successfully as the author was able to replicate the movements of a human arm and these movements were subsequently copied into the robotic arm. However, when using the LMC, the hand was the only part of the human arm that could be detected. Also, the condition of the hand (opened or closed) could be captured properly if only the thumb, the index and the pinky fingers were moved as illustrated in Figure 2.3.1C.
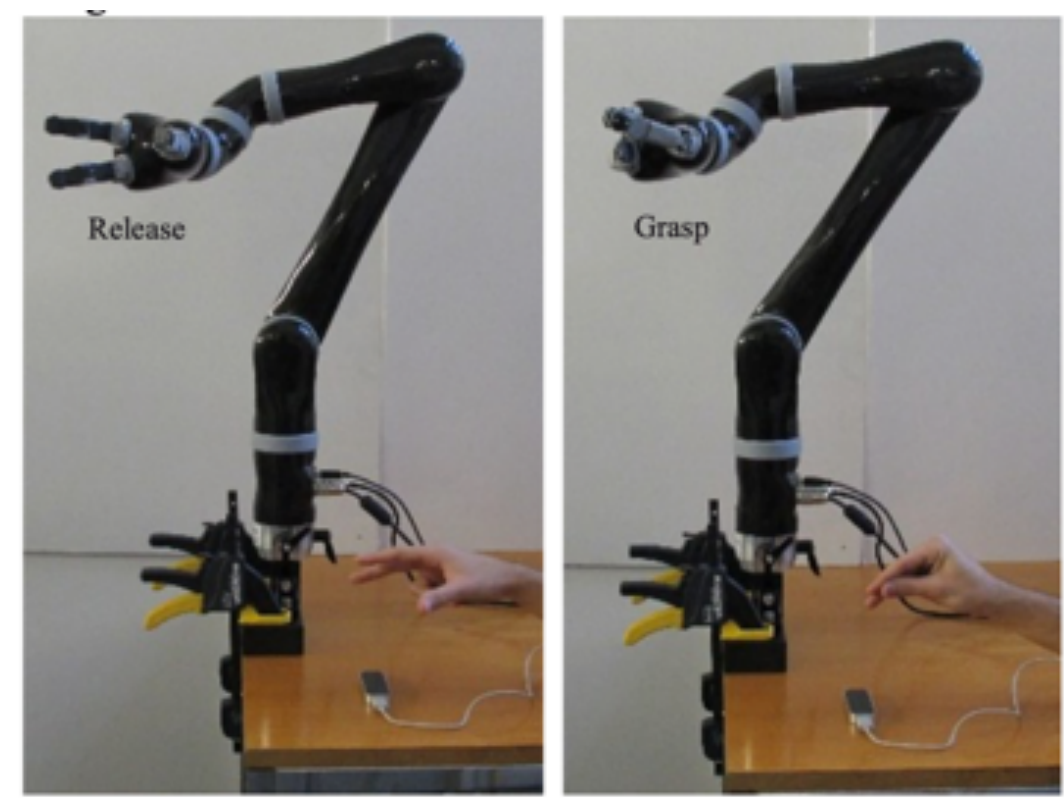

Figure 2.3.1C - Grasp and release operations [93]

\subsubsection{Real-time Glove Control}

By replacing the Kinova joystick with a resistive glove controller, Jacinto demonstrated his robotic arm testing and glove experiments successfully [94]. The JACO robotic arm was used during the experiment. The glove controller, discussed in this research, consisted of three main components: the Inertial Measurement Unit (IMU), flex sensors, and the Arduino Uno microcontroller. A 
similar concept of this methodology can also be found in the following work of [95-99]. In addition to the IMU, three flex sensors are needed to capture the movements of the operator's thumb and first two fingers. Whereas the Arduino Uno microcontroller was used to send and receive data between the flex sensors, IMU, and the robotic arm via Robot Operating System (ROS). ROS was chosen to be the operating system used to support the teleoperation system controlling the robotic arm because there are a lot of useful libraries written ready for programmers to use. In this research, the author was able to complete his tests on the glove controller with simulations of hand movements. However, without the additional JACOSOFT driver, he was not able to control the full robotic arm with the glove controller. A fully assembled glove control used in his research is shown in Figure 2.3.2 below.

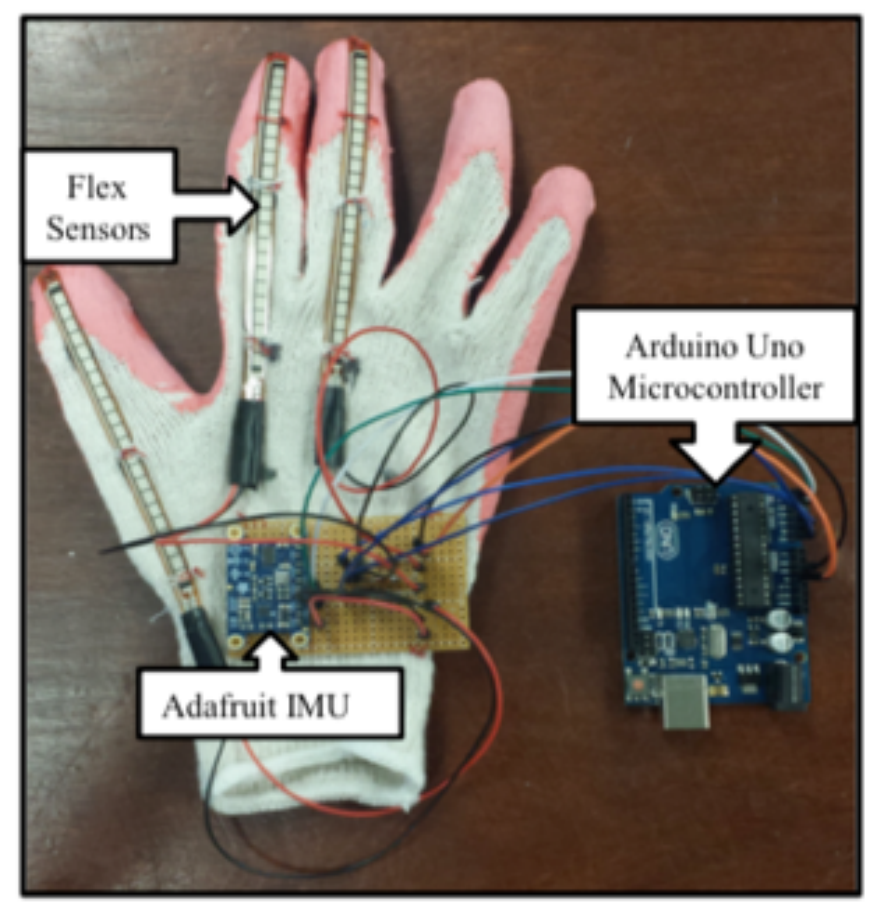

Figure 2.3.2 - Glove controller with flex sensors, IMU and Arduino Uno microcontroller [94] 


\subsubsection{Virtual Joystick}

Another approach, which allows operators to interact with a robotic arm is a virtual joystick, as defined in the work of Palacios [100]. The main purpose of this research was to eliminate the use of mechanical joysticks. Palacios believed this type of controller application is much more convenient than a real-time glove control approach, especially when manipulating various repetitive tasks. Moreover, he claimed, the virtual joystick provides operators with a better flexible manipulation when performing various intricate movements. Below is a graphical user interface screenshot used to control the Kinova JACO arm in all directions.

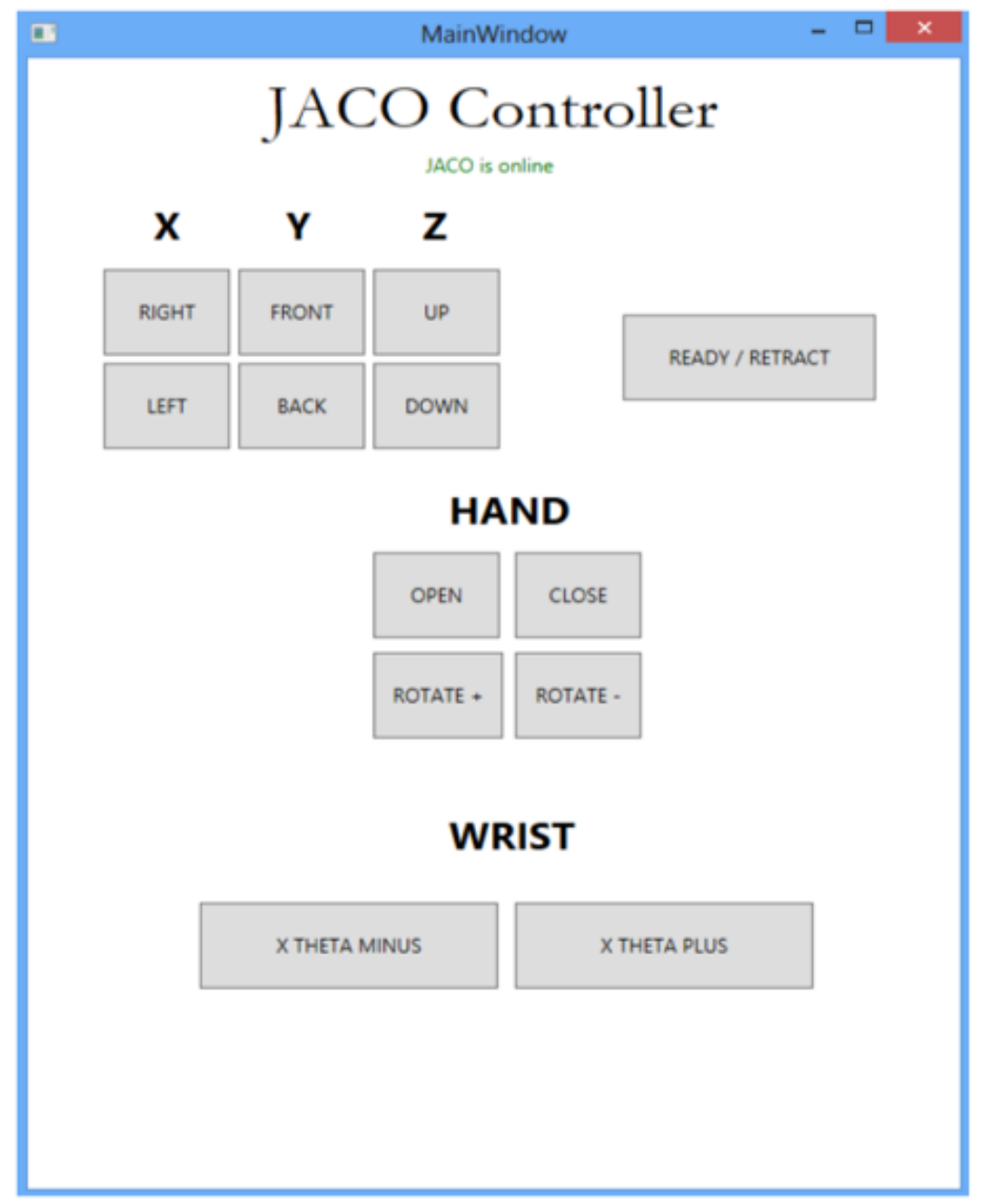

Figure 2.3.3 - Virtual joystick (GUI) [100] 


\subsubsection{Master-Slave Control System}

The idea of teleoperated systems, which has been discussed since the 1970s [101], was invented to help humans with remotely controlling robots from distances. Many sophisticated teleoperation systems have been extensively developed to perform various tasks that do not require autonomy or are too difficult for autonomy to accomplish. These teleoperated systems normally consist of two major parts: a master robot operated by a human and a slave robot working in a real field. The principle of master-slave control systems is to synchronize the movements between the master and slave robots so that motions of the slave robot can be copied exactly from the master one $[102][103]$.

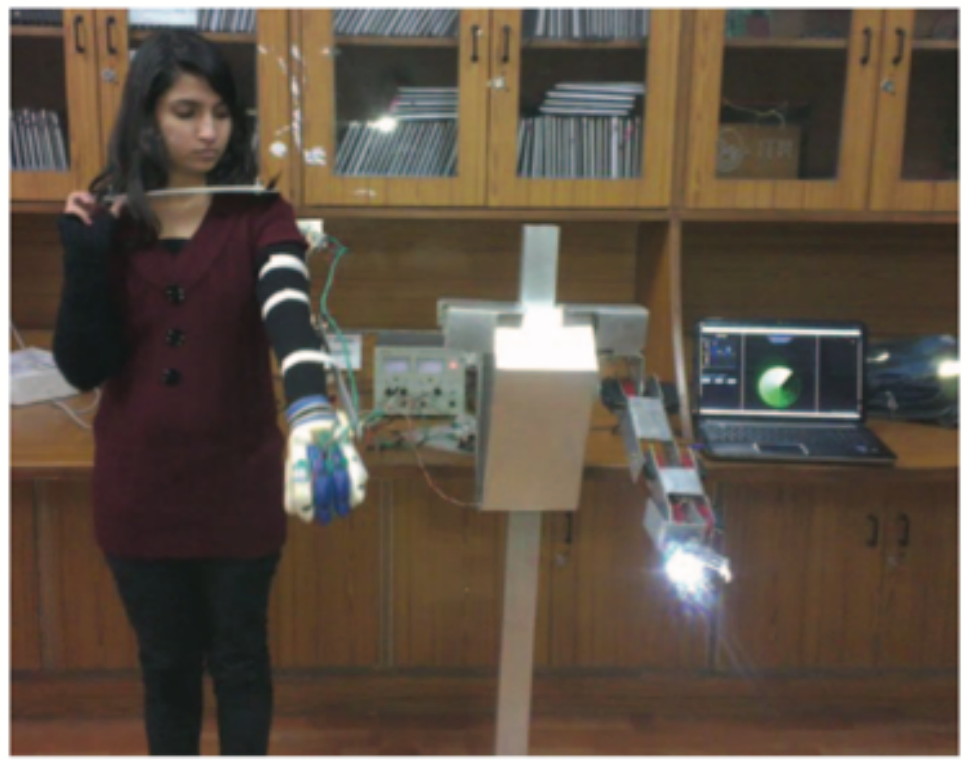

Figure 2.3.4 - A typical master-slave control system [103]

Most of the master-slave control systems [104-106] are based on a haptic interface for remotely controlling slave robots. According to [105], these systems have been found to often be difficult to use and more time consuming due to training requirements. To address this, an enhanced mechanism using the force feedback [107-111] is broadly used in various forms of master-slave 
control systems. For example, in [103] a sensor was attached to the inside of one of the gripper prongs to capture forces applied whenever the gripper was closed. In order to capture the correct position of the master robot (the user's arm), a gyroscope and set of potentiometers were also needed in a control rig (fitted to the user's arm). These potentiometers were mounted on the master robot at the following: the wrist, the elbow, the abduction, and the shoulder joints allowing the entire Human-Machine Interface (HMI) control methodology to mimic the exact movements of the master robot arm. Although these advanced systems have been successfully deployed in real applications; unfortunately, they are complex, expensive, and less intuitive to use due to additional operating control hardware needed on the master robot [112].

\subsection{Fundamental Concepts of Robot Manipulators}

Kinematics is a branch of classical mechanics that describes the motion of joints, bodies (objects), and systems of bodies (groups of objects) without considering the mass of each or the forces that caused the motion [113]. Kinematics is considered as the most important aspect of robot design, control, and simulation. According to [114], robot kinematics studies the relationship between the dimensions and connectivity of kinematic chains and the position, velocity and acceleration of each link in the robot system. Robot kinematics can be separated into two fundamental problems: forward kinematics and inverse kinematics.

\subsubsection{Forward Kinematics}

Forward kinematics, also referred to as direct kinematics, is the process of finding the position and orientation of an end effector relative to its base of a robot given the joint angles and the values of all geometric links [115-117]. Generally, forward kinematics problem is simple to solve as there 
is no complexity deriving the equations. The forward kinematics problem can be easily solved by calculating the transformation describing the position and orientation of the end effector relative to its base, which can be obtained by applying homogeneous matrices of transformations between frames fixed in adjacent links and the algorithm developed by Denavit-Hartenberg (D-H) [118]. To describe the robot kinematics, D-H method uses the following four parameters: $\mathrm{a}_{\mathrm{i}-1}$ (the length of the link), $\alpha_{\mathrm{i}-1}$ (the angle between axes $\mathrm{z}_{\mathrm{i}-1}$ and $\mathrm{z}_{\mathrm{i}}$ measured along $\mathrm{X}_{\mathrm{i}}$ ), $\mathrm{d}_{\mathrm{i}}$ (the link offset) and $\theta_{\mathrm{i}}$ (the joint angle between axes $\mathrm{x}_{\mathrm{i}-1}$ and $\mathrm{x}_{\mathrm{i}}$ measured about $\mathrm{Z}_{\mathrm{i}}$ ), as shown in Figure 2.4.1 below.

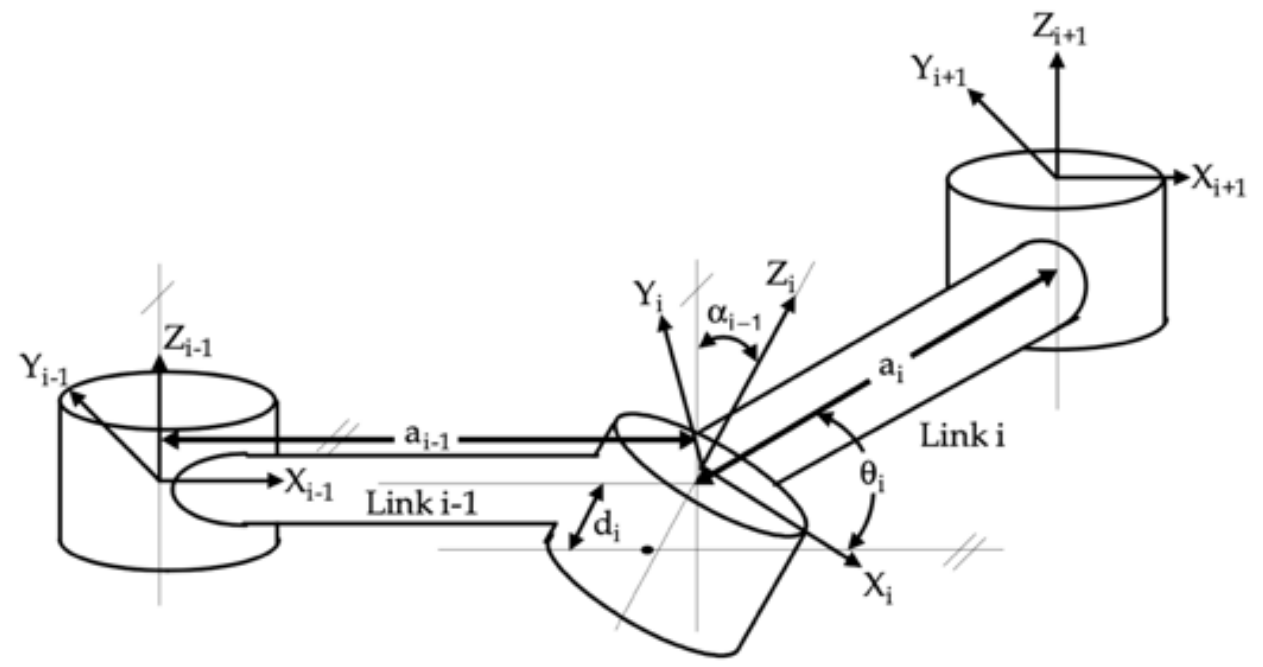

Figure 2.4.1 - Coordinate frame assignment for a general manipulator [119]

According to the D-H representation, transforming from one link to the next link can be done by using the following basic transformations: rotations and translations, presented by equation (1).

$$
\begin{aligned}
& { }_{\mathrm{i}}^{\mathrm{i}-1} \mathrm{~T}=\mathrm{R}_{\mathrm{x}}\left(\alpha_{\mathrm{i}-1}\right) \mathrm{D}_{\mathrm{x}}\left(\mathrm{a}_{\mathrm{i}-1}\right) \mathrm{R}_{\mathrm{z}}\left(\theta_{\mathrm{i}}\right) \mathrm{Q}_{\mathrm{i}}\left(\mathrm{d}_{\mathrm{i}}\right) \\
& =\left[\begin{array}{cccc}
1 & 0 & 0 & 0 \\
0 & c \alpha_{i-1} & -s \alpha_{i-1} & 0 \\
0 & s \alpha_{i-1} & c \alpha_{i-1} & 0 \\
0 & 0 & 0 & 1
\end{array}\right]\left[\left[\begin{array}{cccc}
1 & 0 & 0 & \mathrm{a}_{i-1} \\
0 & 1 & 0 & 0 \\
0 & 0 & 1 & 0 \\
0 & 0 & 0 & 1
\end{array}\right]\right]\left[\begin{array}{cccc}
c \theta_{i} & -s \theta_{i} & 0 & 0 \\
s \theta_{i} & c \theta_{i} & 0 & 0 \\
0 & 0 & 1 & 0 \\
0 & 0 & 0 & 1
\end{array}\right]\left[\begin{array}{cccc}
1 & 0 & 0 & 0 \\
0 & 1 & 0 & 0 \\
0 & 0 & 1 & \mathrm{~d}_{i} \\
0 & 0 & 0 & 1
\end{array}\right] \\
& =\left[\begin{array}{cccc}
c \theta_{i} & -s \theta_{i} & 0 & \mathrm{a}_{i-1} \\
s \theta_{i} c \alpha_{i-1} & c \theta_{i} c \alpha_{i-1} & -s \alpha_{i-1} & -s \alpha_{i-1} \mathrm{~d}_{i} \\
s \theta_{i} s \alpha_{i-1} & c \theta_{i} s \alpha_{i-1} & c \alpha_{i-1} & c \alpha_{i-1} \mathrm{~d}_{i} \\
0 & 0 & 0 & 1
\end{array}\right]
\end{aligned}
$$


where $R_{x}$ and $R_{z}$ denote rotations, $D_{x}$ and $Q_{i}$ present translations, and $c \theta_{i}$ and $s \theta_{i}$ are short forms for $\cos \theta_{\mathrm{i}}$ and $\sin \theta_{\mathrm{i}}$, respectively. For a robot arm consisting of six joints, the forward kinematics of the end effector with respect to its base frame can be defined by multiplying all of the ${ }^{\mathrm{i}-1} \mathrm{~T}$ matrices as follows:

$$
{ }_{6}^{0} \mathrm{~T}={ }_{1}^{0} \mathrm{~T}\left(\mathrm{q}_{1}\right){ }_{2}^{1} \mathrm{~T}\left(\mathrm{q}_{2}\right) \ldots{ }_{6}^{5} \mathrm{~T}\left(\mathrm{q}_{6}\right)
$$

where $\mathrm{q}_{\mathrm{i}}$ is the joint variable $(\mathrm{i}=1,2, \ldots 6)$.

In the equation (1) above, two parameters ( $\mathrm{a}_{\mathrm{i}-1}$ and $\left.\alpha_{\mathrm{i}-1}\right)$ are always fixed and used to describe the link itself whereas the other two parameters presenting the link connection of the neighbour's link are variable. Fundamentally, there are two types of joint: revolute joint and prismatic joint which can be summarized in detail in Table 2.4.1.

Table 2.4.1 - Four D-H parameters [120]

\begin{tabular}{|c|c|c|}
\hline Parameter & Revolute joint & Prismatic joint \\
\hline Link length $\left(\mathrm{a}_{\mathrm{i}-1}\right)$ & constant & constant \\
\hline Twist angle $\left(\alpha_{\mathrm{i}-1}\right)$ & constant & constant \\
\hline Link offset $\left(\mathrm{d}_{\mathrm{i}}\right)$ & constant & variable \\
\hline Joint angle $\left(\theta_{\mathrm{i}}\right)$ & variable & constant \\
\hline
\end{tabular}




\subsubsection{Inverse Kinematics}

With the inverse kinematics problem, it generally takes a very long time to solve the values of joint angles given the position and orientation of an end effector relative to the base and the length of all geometric links [121-124]. In other words, the inverse kinematics problem can be defined as a conversion of the position and orientation of the end effector from Cartesian space to joint space [119]. There are numerous ways for finding the inverse kinematics solution; two common approaches have been used to solve the reverse kinematics problem are geometric and algebraic.

\subsubsection{Degree(s) of Freedom}

The degree of freedom (DOF) has been defined as the number of independent variables required to define the position of a typical rigid object in space [125][126]. In other words, DOF is used to define the number of directions in which a particular object can move. In the following example, the position of a single engine moving along a track has one degree of freedom because its position is defined by the distance along the track. Whereas the motion of a ship at sea as depicted in Figure 2.4.3 has six degrees of freedom as it could move up and down, left and right, or forward and backward. In addition, it could swivel left and right (yawing), or tilt forward and backward (pitching), or pivot side to side (rolling).

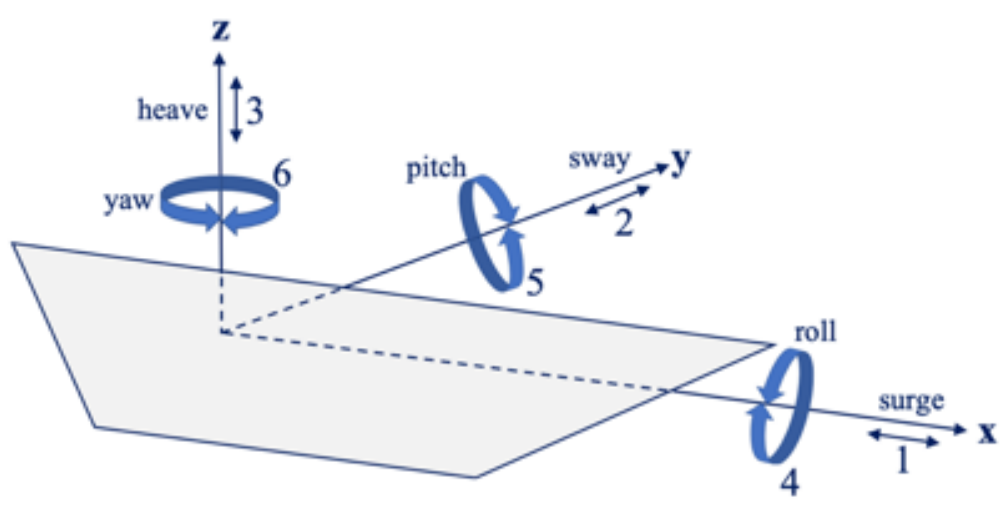

Figure 2.4.3 - The six degrees of freedom of movement of a ship 
In the example of 6-DOF in a motion of a ship above, surge, sway, and heave are translational motions whereas roll, pitch, and yaw are rotational motions caused by sea waves and might be reduced by internal and/or external forces (propellers, rudders) and environments conditions (wind and sea currents). Similarly, the trajectory of an airplane in flight has three degrees of freedom and its attitude along the trajectory has another three degrees of freedom resulting in a total of six degrees of freedom. In the following example [127], the robotic arms used to implement and test real-time collision avoidance in this research which are similar to a human arm have seven degrees of freedom.

\subsection{Standardized Test Methods}

The U.S. Department of Homeland Security, Science and Technology Directorate (DHS S\&T) engaged a long term partnership with the National Institute of Standards and Technology (NIST) to develop a series of standardized tests for the purposes of collecting capability data concerning response robots and providing significant data comparison regarding associated apparatuses, procedures, and performance metrics [128-131]. Many emergency responders have to risk their lives by dealing with a variety of extreme hazards for which remotely operated robots should be deployed to protect the public. When dealing with hidden hazards such as suspected IEDs while searching survivors in collapsed buildings or compromised structures, responders want to start and stay remotely. For this reason, various capable robotic systems have been developed and tested from safe stand-off distances to provide situational awareness when performing dexterous object manipulation tasks.

According to [132], the main goal of improving safety related manipulation tasks is to find the effective and safe way of remotely deploying various robotic systems by measuring key capabilities of robots for emergency response and various other hazardous applications. In order 
to achieve this, many comparisons of different robotic systems have been conducted by NIST using a set of standardized test methods to capture the capability of remote deployments over a significant number of repetitions. If any robot operator cannot successfully complete the specified test methods, they will not be allowed to perform the operational tasks during deployments.

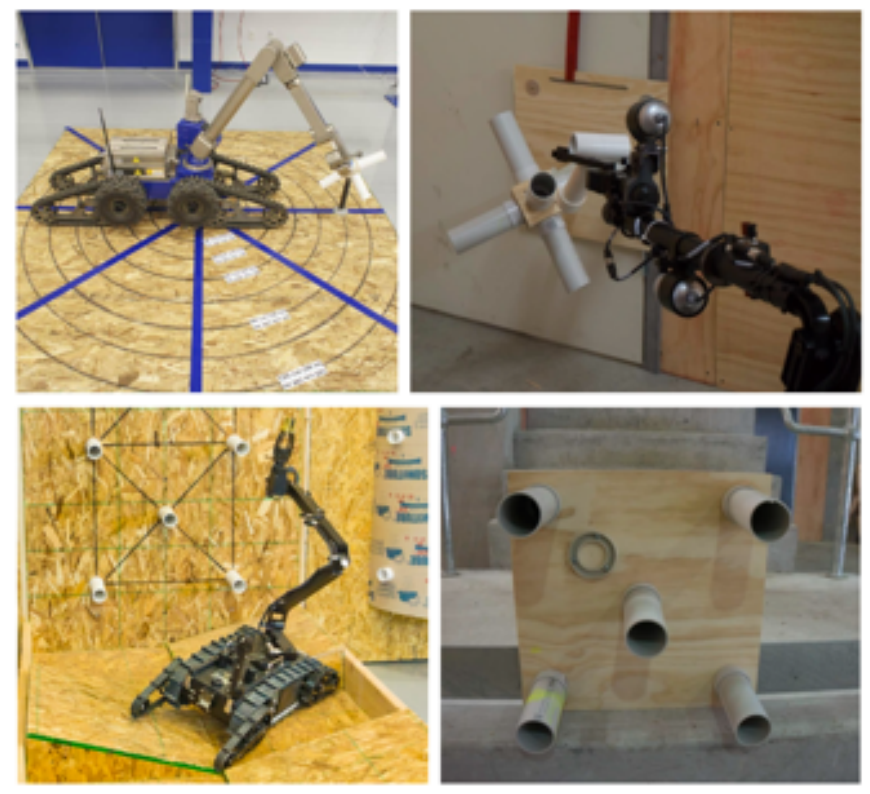

Figure 2.5 - Examples of test methods conducted by NIST in Gaithersburg, MD, USA [133]

In general, each test requirement must have an associated metric as a way to measure the capability of any particular robotic system used during deployments. These standard metrics can be as simple as such elapsed time or a number of repetitions with time per task as a secondary measure of efficiency. Test trials are usually in between 10-30 repetitions to statistically achieve significant comparisons. Remotely operated response robots controlled from a remote operator station used in these tests include various ground vehicles, unmanned aerial systems and/or aquatic systems, etc. as described in section 2.2. Whereas the test apparatuses associated with these test methods 
used by these robots to perform a number of repeatable tasks are typically fabricated using various inexpensive and proliferate widely materials.

During deployments, robot operators are expected to perform a specific requirement such as climbing over a wall, or simply getting over variable terrains, assorted obstacles, confined areas, etc. chosen by different responders, robot developers, procurement sponsors, or other sources [134]. By conducting these standardized test methods, as a final outcome, a database of useful test results can be generated and subsequently used to determine the baseline capabilities of many different tested robots. Further, this important data about robot capabilities is useful for guiding several robot procurements and can be used to support the standardization process. 


\subsection{Summary}

This chapter discussed the important capabilities of response robots and several ways of remotely controlling a robotic arm by using various techniques. Although many advanced teleoperation systems were discussed with using different control techniques, these systems seem to be either complex or expensive due to the extra interface or the additional driver required to enhance the performance of the systems. Most importantly, the complexity of additional operating control hardware needed on the master robot which is less intuitive for operators to manipulate the movement of the slave robot at a distance and has not been effectively addressed as yet. In addition, this chapter also presented several fundamental concepts of robot manipulators as well as various test methods that have been developed and run by NIST to quantify key capabilities of emergency response robots. 


\section{Chapter 3}

\section{Technical Approach}

\subsection{Introduction}

Much research has been done in the field of robot arms to help humans with performing dexterous operations from remote locations. In this chapter, we add to that body of knowledge by describing the details of general control principles to manipulate robotic arms using the LF approach. We also present an overview of several fundamental concepts for controlling robot manipulators. There are several ways for achieving control. A common way is to use some form of joystick controller. We propose that control can also be achieved more intuitively by a control integration approach of one arm mimicking another arm's position that we define as the "Leader-Follower approach". In our experiments, we will use twin Kinova robot arms to both demonstrate control and measure its efficacy in relation to other control strategies.

\subsection{Control Integration}

This section describes several options that operators can use when controlling a Kinova robot arm. Fundamentally, as with many commercially available robot arms, operators can control the arm by either using a Kinova joystick or writing software to control via the Kinova Application Interface (API) [135-137]. With a standard joystick, robotic actuators can be controlled by either end effector position or actuators' angular position. When operators control movements of the arm either by translating the position of the end effector or rotating the orientation of the end effector around its reference point, the control over the arm is considered to be Cartesian. As a result, 
movements of all joints are adjusted accordingly for any given command. Whereas the robot is placed in "Angular mode", operators can command each specific actuator's rotation rather than its position. In the first option, operators can connect the standard joystick provided to the joystick port and control the arm in Cartesian velocity by default or in Angular velocity if angular control is enabled. Details of how to control the robot arm using a joystick are presented later in section 3.3. Alternatively, operators can install and run the Kinova software development kit (SDK) Development Center on a personal computer and write custom software to control the arm via the API.

When choosing the API to control the arm, operators can use either one of the following two options:

1. Kinova software control: This option allows operators to manually control the arm via a graphical user interface (GUI) by running any of the following software panels: the Development Center and the Torque Console. This option is quite useful as operators can manipulate the arm in position, velocity, and trajectories. In addition, operators can send trajectories, monitor the current robot's state, activate, and switch between Cartesian and angular control.

2. API control: Operators can control the arm by using a library of functions written in $\mathrm{C}++$ provided by Kinova. This library of functions is also referred to the Kinova API which can be downloaded from Kinova's website as part of the SDK. Currently, the Kinova API is available on both Windows and Ubuntu. Optionally, Kinova also offers another way for developers who prefer to manipulate the arm through a ROS interface. 
Of course, operators can also directly control the arm by moving its various parts manually. In other words, operators are able to move the arm freely by manually pushing or pulling on some link or even moving the end-effector.

\subsection{Joystick Control}

To start, operators must proceed with the following steps:

1. Turn on the device by switching the $O N / O F F$ button on the base of the arm and wait until the joystick ready light is fully on.

2. Operators should then wait until the green lights on the joystick controller stop flashing before putting the arm in its HOME position by holding on the HOME/RETRACTED button. If operators hold the HOME/RETRACTED button again, the arm will move to its RETRACTED position. These two factory default positions are pre-set: the HOME and the RETRACTED position (Figure 3.3.1). When the arm is in its HOME position, it is ready to receive a command from the joystick. When the arm is in its RETRACTED position, it is in standby mode; therefore, most of the joystick features are not functional.

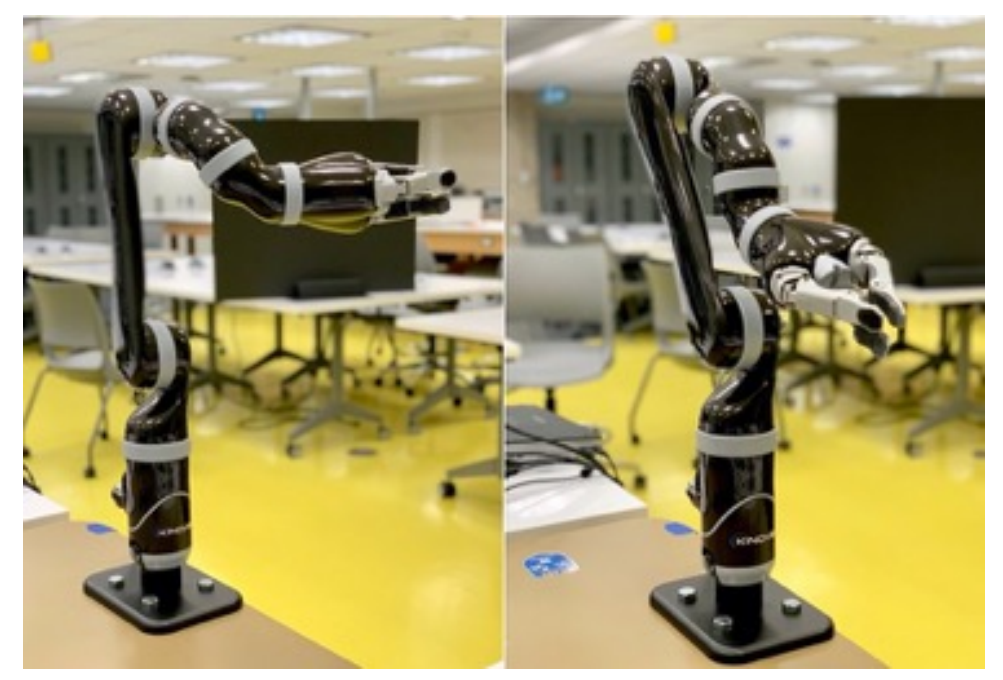

Figure 3.3.1 - Kinova $\mathrm{MICO}^{2}$ arm in HOME (left) and RETRACTED (right) position 
When powering the arm on, it is set in Cartesian mode which allows operators to move the arm in 3-Axis operation mode. It can easily be switched into 2-Axis operation mode by holding the ON/OFF button on the joystick for 2 seconds. While the arm is in 3-Axis operation mode, to activate:

- Finger control mode, operators should press on Button $A$.

- Wrist control mode, operators should press on Button $B$.

To enable Translation control mode, simply press on Button $B$ again. While in Wrist mode, operators can also activate the Drinking mode by simply pressing on Button 1 or press it again to deactivate the Drinking mode.

When the arm is set in 2-Axis mode, operators can press on Button 3 to enable the control for Translation-Z and the wrist or simply press on Button 3 again to bring back to $\mathrm{X}$ and $\mathrm{Y}$ translation. In order to activate Wrist control mode, operators should press on Button 2 or press it again to switch to Finger control mode. Similarly, when the arm is in 3-Axis mode, while in Wrist mode, operators can also activate/deactivate the Drinking mode by simply pressing on Button 1 . To help operators better understand how to operate the joystick for each mode, detailed instructions are summarized in Figure 3.3.2 and Table 3.3. Operators can also figure out which mode is currently in use by checking visual retroaction such as:

- Blue lights: Feedback on control mode (see Appendix A for details)

- Green lights: Feedback on arm power

- Red lights: Feedback on error 


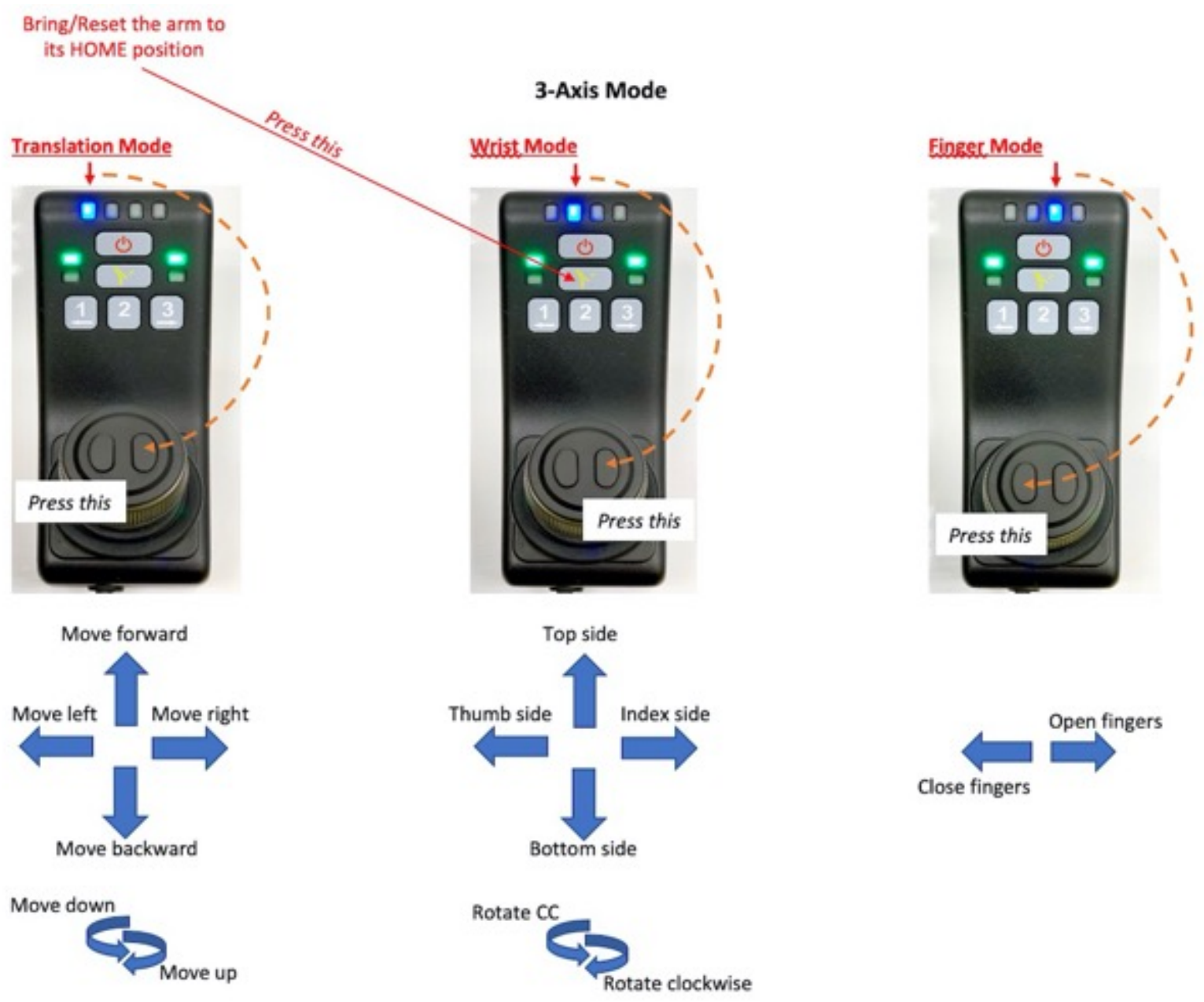

Figure 3.3.2 - 3-Axis operation mode 
Table 3.3 - 2-Axis operation mode

\begin{tabular}{|c|c|}
\hline Joystick Movement & MICO $^{2}$ arm Movement \\
\hline \multicolumn{2}{|c|}{ Translation-X \& Translation-Y } \\
\hline Incline front & Gripper moves forward \\
\hline Incline back & Gripper moves backward \\
\hline Incline left & Gripper moves left \\
\hline Incline right & Gripper moves right \\
\hline \multicolumn{2}{|c|}{ Translation-Z \& Wrist Rotation } \\
\hline Incline front & Gripper moves up \\
\hline Incline back & Gripper moves down \\
\hline Incline left & Wrist rotation clockwise \\
\hline Incline right & Wrist rotation counter-clockwise \\
\hline \multicolumn{2}{|c|}{ Wrist Mode } \\
\hline Incline front & Vertical orientation - Bottom side \\
\hline Incline back & Vertical orientation - Top side \\
\hline Incline left & Lateral orientation - Index side \\
\hline Incline right & Lateral orientation - Thumb side \\
\hline \multicolumn{2}{|c|}{ Finger Mode } \\
\hline Incline left & Close 3 Fingers \\
\hline Incline right & Open 3 Fingers \\
\hline Incline back & Close 2 Fingers \\
\hline Incline front & Open 2 Fingers \\
\hline
\end{tabular}


By default, the Kinova joystick is set to be controlled in Cartesian mode; operators can individually control each actuator or each of the fingers when switching to Angular mode. This change can be done from the settings in the Kinova SDK Development Center. While the arm is in Angular mode, operators can press on either Button $A$ or Button $B$ to change the status of each blue light appearing on the joystick controller or simply press on Button 1 to turn the $3^{\text {rd }}$ blue light on which enables Finger control mode. Complete details of joystick movements for each specific actuator and each of the fingers can be found in Figure 3.3.3.

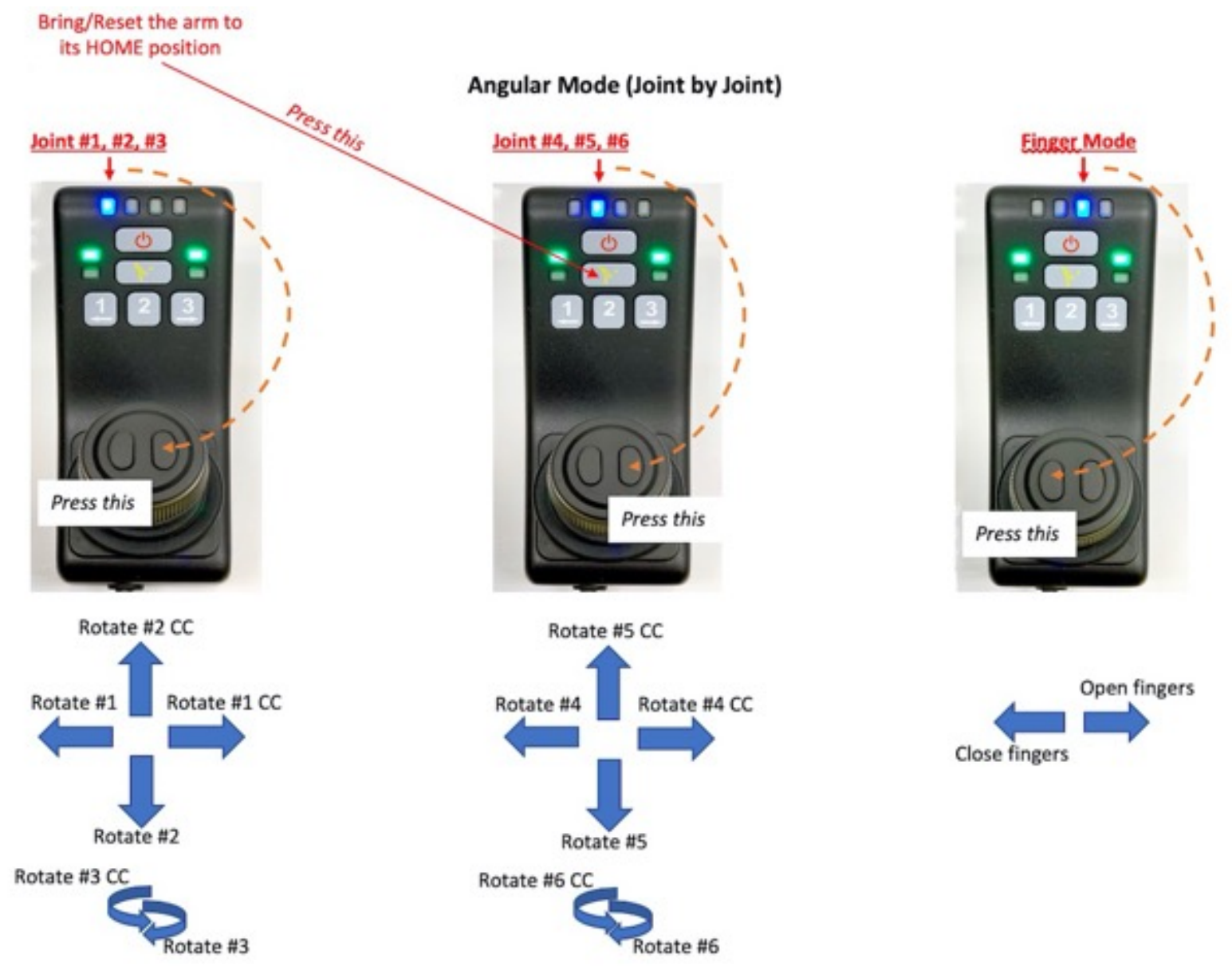

Figure 3.3.3 - Angular mode 


\subsection{Leader-Follower Approach}

Many robot systems have been built to assist humans with performing hazardous tasks. According to [138-140], many different kinds of response robots have been developed to help humans with performing several hazardous tasks without putting humans in danger. These response robots are very useful in specific scenarios such as explosive or other hazardous material manipulation, as humans do not have to be near the dangerous environments and can control the robots from safe standoff distances.

The Leader-Follower method is introduced in this section. With or without using a Kinova joystick, the LF method allows operators to manipulate the movement of one arm remotely while having another remote arm mimics this motion at a distance. We believe the LF method is advantageous for safety related manipulation tasks if high-fidelity control links can be established between the operator and the remote arm. As there is no special training required or special knowledge about robot control, the method should reduce the cognitive load of the arm's operator. However, in our setup, the LF method does require some quick training for operators as they are not able to directly see actual movements of the remote arm but through a quad display providing views of the apparatus from 3 cameras. To achieve the best performance with the LF method, robot arm operators must address the following challenges:

1. Accuracy and practicability

2. Fixed quad displays and spurious states

We argue that the LF method provides operators with a more flexible manipulation when performing various complicated movements; each motion of the remote arm can be synchronized precisely with the operator arm. The implementation of this method is done in $\mathrm{C}++$ so that it can receive data input from one $\mathrm{MICO}^{2}$ arm, known as the operator arm, in Cartesian coordinates. 
These values are scaled respectively before being translated into a new set of $\mathrm{x}, \mathrm{y}, \mathrm{z}$ coordinates required to move the second $\mathrm{MICO}^{2}$ arm--known as the remote arm. Since the $\mathrm{MICO}^{2}$ arm can be controlled in all directions; therefore, moving all joints quickly and accurately is considered as one of the main challenges for most operators.

The LF method also requires that operators interpret the situation of the remote arm through fixed camera views during experiments. With the information obtained through a 4 quadrant (quad) display, operators might experience some difficulties trying to replicate movements between the operator arm and the remote arm given that the operator will be looking at a fixed representation of the situation of the remote arm in the fixed quad display. We believe, during the training process, operators should concentrate on how to move the individual joints on the operator arm to achieve the results they want on the remote arm in order to learn how to achieve good performance results. However, there are cases where some actuators on the arm may exceed their range limits. In these cases, an arm may enter a spurious state and stop moving altogether.

\subsection{Test Methodologies}

The $\mathrm{MICO}^{2}$ arm is mainly designed to be used in indoor environments. In this section, we discuss several different test methods which have been developed for identifying the efficacy of the LF control metaphor when dealing with complex manipulations. These test methods have been adapted from a standard set of test methods being developed by NIST, as described in chapter 2 , and adopted by the American Society for Testing and Materials (ASTM) standards body. Many of these test methods have been adopted by first responder organizations to test robot arms against a standard methodology to support comparisons between robotic systems from competing manufacturers. 


\subsubsection{Standard Metrics}

The main purpose of these test methods is to quantitatively evaluate the manipulator grasping and dropping capability of a remotely teleoperated robot arm operating on an indoor environment. The metrics we are interested in are the average time needed per test mode and the number of grasped pick and drop objects between the three test modes. Prior to each test mode, operators are given about 15 minutes to train themselves to be familiar with operating the system. From reported findings and comparisons of different test methods, we can use these metrics to evaluate the best way of deploying robot arms for the object ${ }^{1}$ manipulation task by comparing the average time per task and the number of successful completes per test mode. During the tests, we also record the number of times operators fail to pick or drop objects into the target location for each run as well as the total time needed to complete each test mode. For each test mode, to avoid the situations when operators take too long to complete, we limit the total time allowance to be 15 minutes. In addition, any hardware or software failtures, camera issues, or signal problems, etc. are considered as fault conditions while running the tests.

\subsubsection{Apparatus}

The apparatus for our test methods use two stations separated away by a short distance. The operator station has one robot arm and the remote station has another robot arm with which pick and drop tasks are performed. Each robot arm is inserted on top of a mounting post which is firmly attached to a mounting plate as shown in Figure 3.5.2A. The entire mounting kit is then affixed to a flat surface so that the arm is fixed in such a way that its base cannot fall during operations. The robot support holds the robot securely in place. It is fixed to a flat surface and the robot base is

\footnotetext{
${ }^{1}$ Objects in our experiments are abstractions of possible objects to be manipulated in a real-world scenario. Typically, for EDU/CBRNE operations, these might be components, packaging or objects around an IED.
} 
mounted onto a mounting post on the adapter. The robot base is then secured using a collar mechanism and three locking knobs (Figure 3.5.2B).

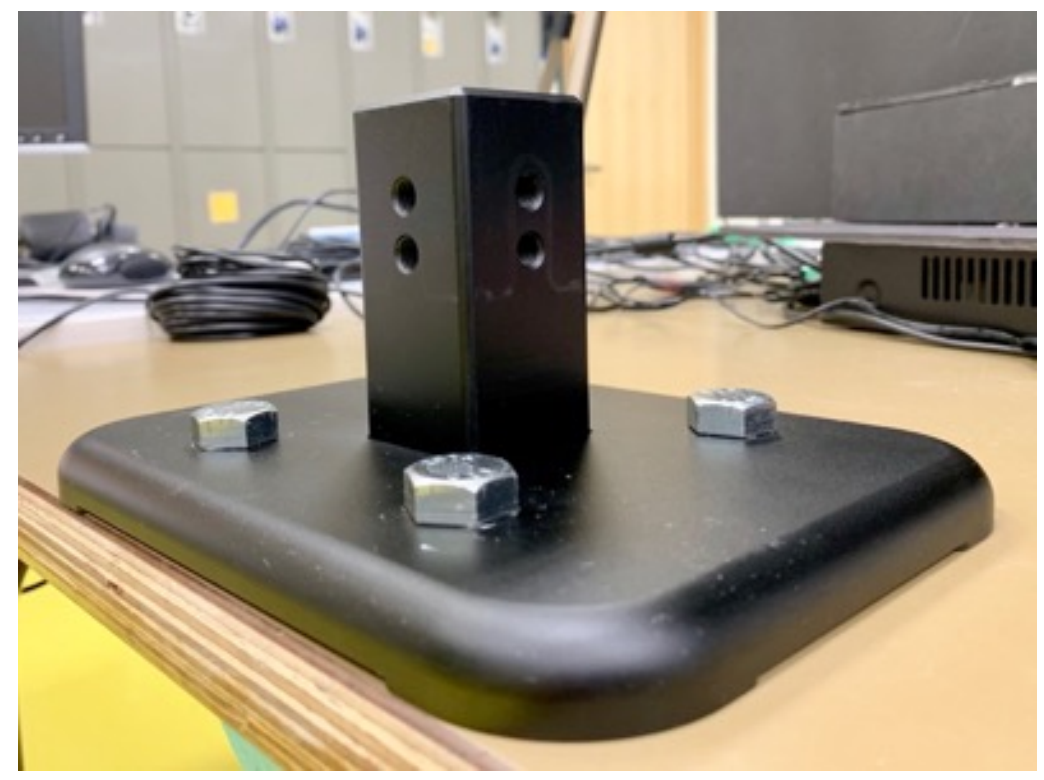

Figure 3.5.2A - Mounting plate used for inserting a robot arm on top

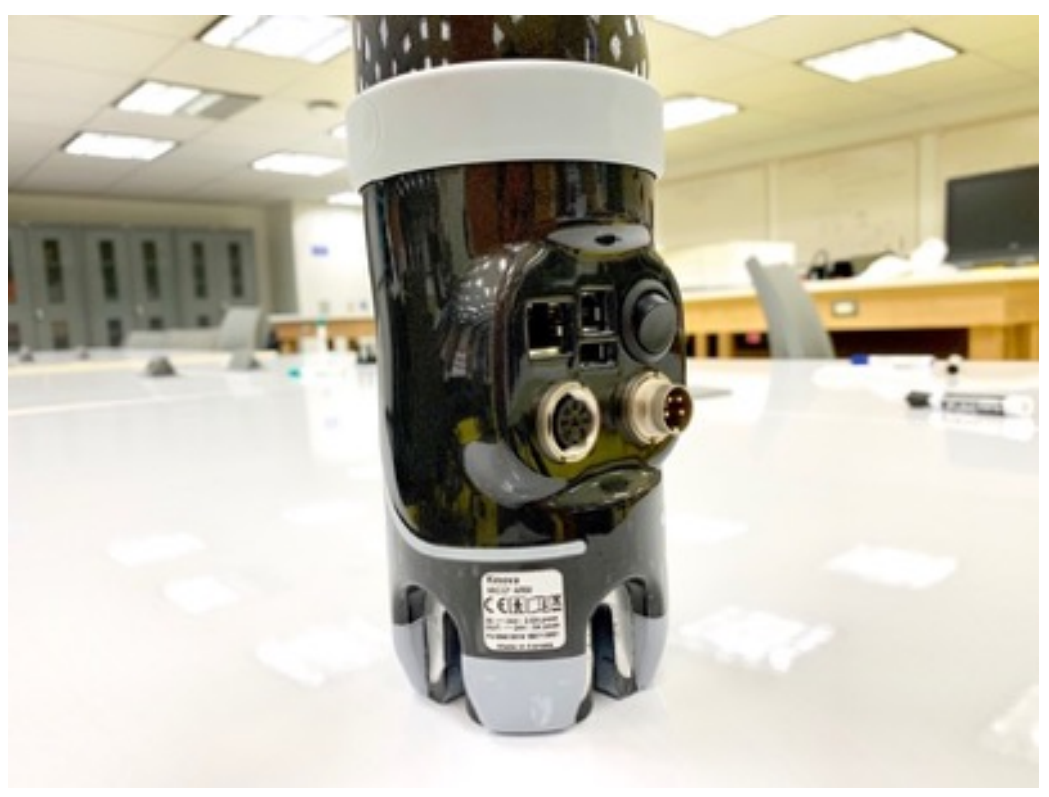

Figure 3.5.2B - Base of the $\mathrm{MICO}^{2}$ arm before being mounted on the mounting plate 
Kinova has made many different versions of robot arms which can be used in many assistive applications or mounted on power wheelchairs. In our research, we use in a 6 DOF curved wrist $\mathrm{MICO}^{2}$ arm.

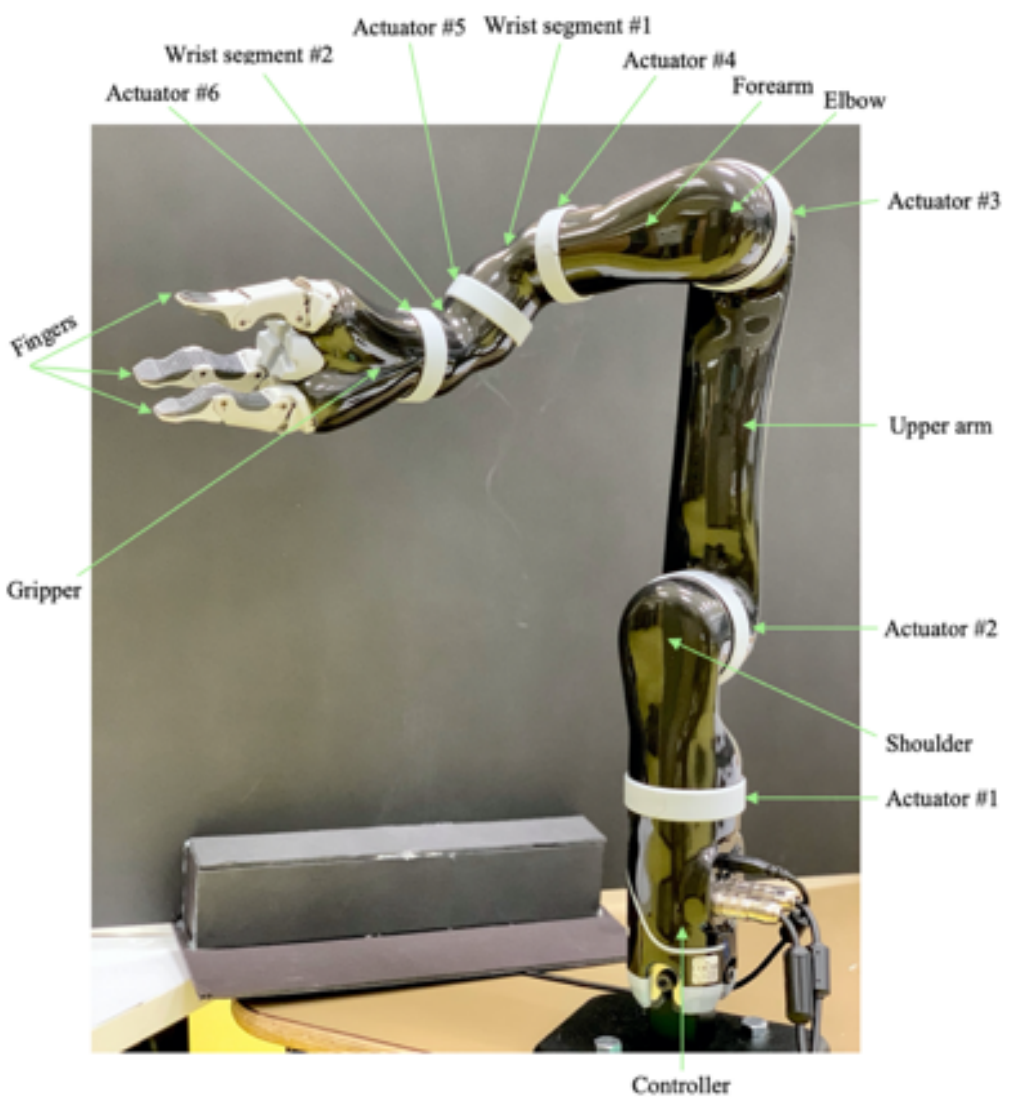

Figure 3.5.2C - Kinova 6 DOF curved wrist $\mathrm{MICO}^{2}$ arm part ID

As illustrated in Figure 3.5.2C above, this robot arm is composed of 6 interconnected carbon fibre links which are connected to joints and driven by aluminum actuators. These joints are numbered from the base to the end effector of a robot in sequential order. Each of these joint-links forms a DOF. As a human arm, this robot arm has a shoulder, an elbow, a wrist, and even fingers. This $\mathrm{MICO}^{2}$ arm is capable of lifting different sizes and types of light objects up to $1.3 \mathrm{~kg}$ and can easily be mounted on either a mobile platform or a fix station. 
The end effector of the $\mathrm{MICO}^{2}$ arm consists of three plastic fingers which can be operated independently. Typically, the base is used to position the end effector (or gripper $^{2}$ ). The end effector is commonly attached to the base using either a spherical or curved wrist whereas the wrist is used to orient the gripper at its desired position. The gripper of the $\mathrm{MICO}^{2}$ arm used in this thesis, as shown in Figure 3.5.2D, offers the maximum versatility and flexibility for picking up different objects of various shapes such as a bottle of water, an orange, or a pair of glasses, etc.

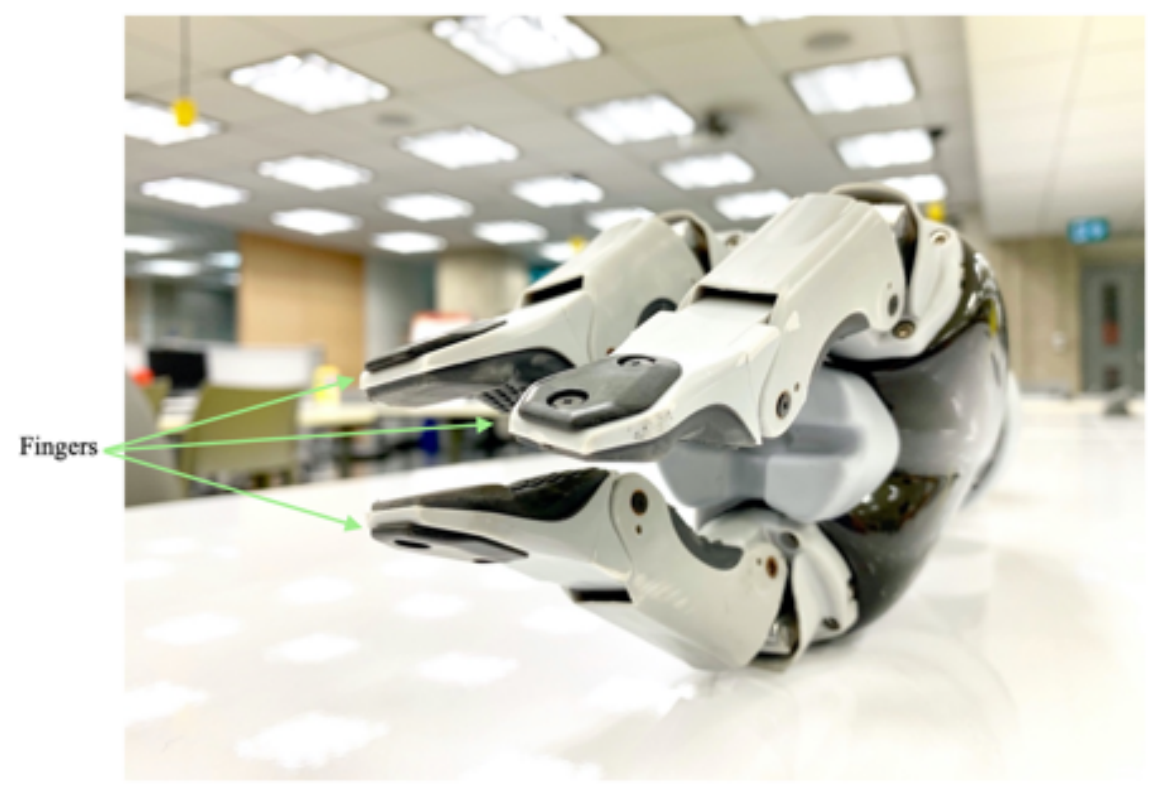

Figure 3.5.2D - Gripper of $\mathrm{MICO}^{2}$ arm with three fingers

${ }^{2}$ Note: The terms end effector and gripper are used interchangeably throughout this report. 
The objects we use in our tests to evaluate the manipulator grasping and dropping capability are lightweight plastic pipes shown in Figure 3.5.2E.

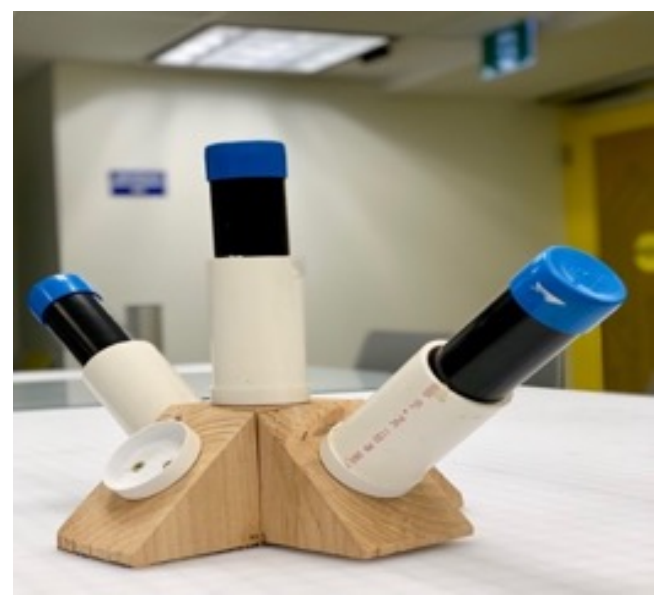

Figure 3.5.2E - Lightweight objects used for grasping and dropping during evaluation exercises

To setup the testing environment as similar as the testing scenarios found during Response Robot Evaluation Exercises developed by NIST, we use a custom divider as shown in Figure 3.5.2F to separate the two robot arms so that operators cannot directly see the results of their control on the remote arm.

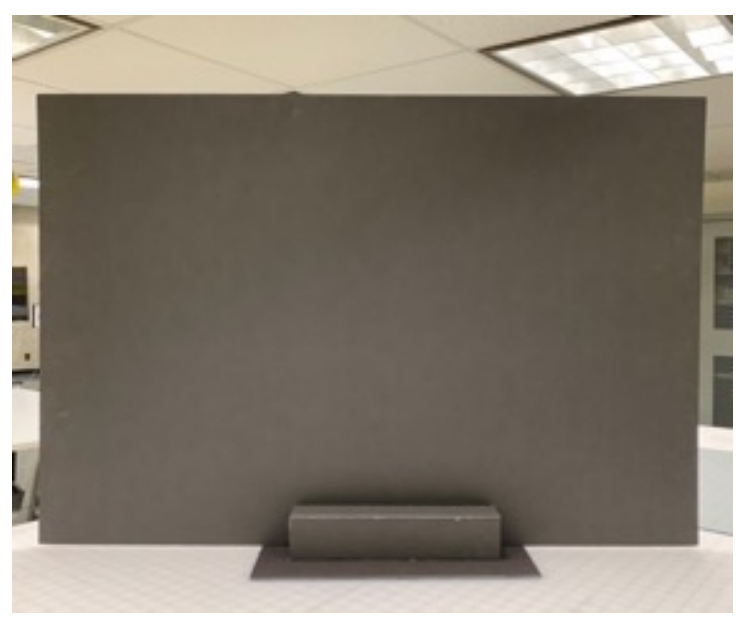

Figure 3.5.2F - Custom divider used to separate two robot arms into two places 
The goal of our research is to determine the efficacy of a control strategy involving one arm being manually moved around to control another arm performing a manipulation task. As the manipulation is to take place remotely, the local scene must be depicted via a camera apparatus. To provide visual telepresence feedback to operators during operations, our cameras are set up at different spots $^{3}$ at the remote station as typically used in evaluating response robots employing 3 cameras [141-143]. One camera is used to capture most of the motion of the arm from a top view provided by a tripod positioned behind the arm. The second camera is mounted beside the gripper (Figure 3.5.2G), providing a clear view of the end-effector in use. There is an additional camera set up on the right side of the arm which is aimed at the target objects.

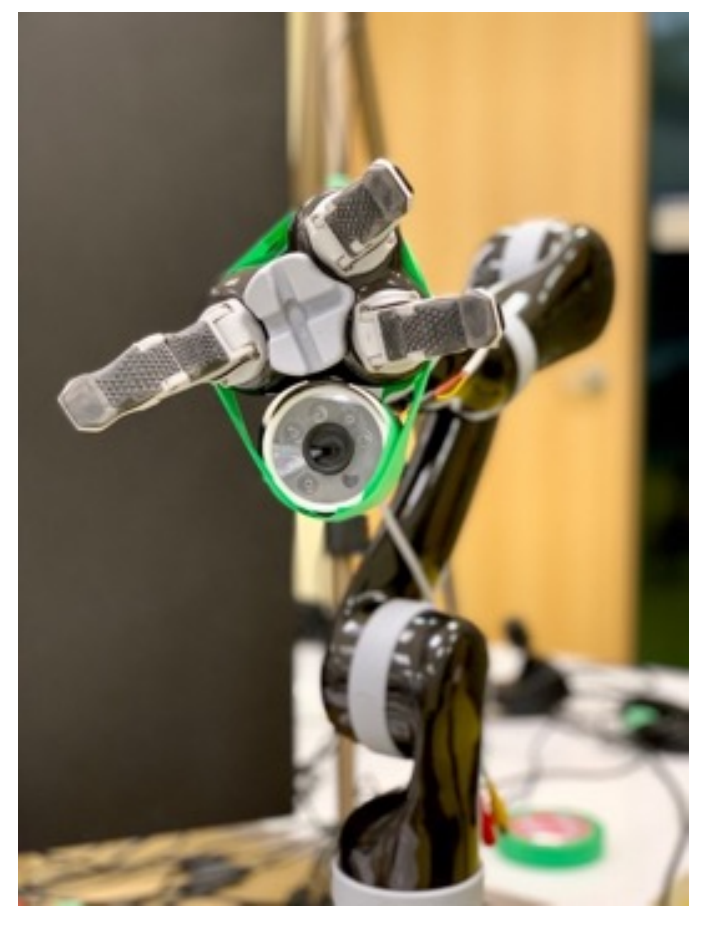

Figure 3.5.2G - Bullet camera attached to the gripper wrist

\footnotetext{
${ }^{3}$ See Appendix B for detail
} 
The following interconnection diagram shows how the teleoperation system can be used to directly control the remote arm with a standard joystick either in 3-Axis mode or in Angular mode or physically control the operator arm from a distance.

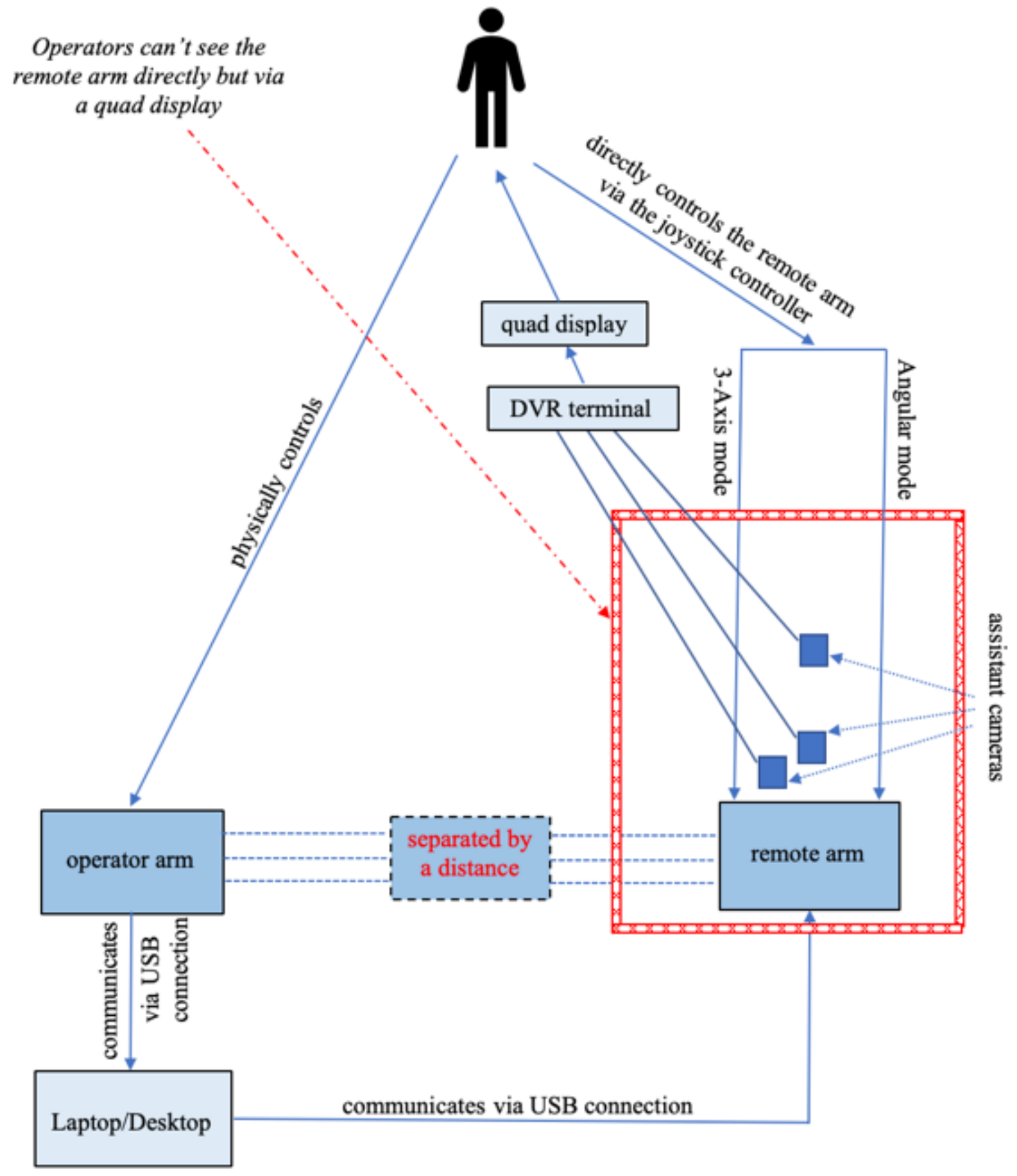

Figure 3.5.2H - Interconnection diagram illustrates the experimental setup 


\subsubsection{Testing Procedures}

The proposed standard metrics for the $\mathrm{MICO}^{2}$ arm do not cover all possible test scenarios found during Response Robot Evaluation Exercises developed by NIST but rather the proposed tests can be used to evaluate and benchmark different levels of difficulty. To evaluate and compare the relative effectiveness of various ways of controlling robot arms, we have elected three different test modes: joystick control in 3-Axis mode, joystick control in Angular mode, and custom software using the API control. Each operator is assigned a test mode randomly to get started. Depending on the test mode assigned, the timer is set as soon as operators start moving the operator arm if API control is assigned or the remote arm if joystick control is assigned. Operators are required to grasp one of the three objects with the gripper and drop each into a designed location. There is an empty container which is set up near by the remote arm and used as a target location for each run. The timer is ended as soon as operators finish transferring each object into the target location. For each test run, we log the total time needed to complete the task as well as any feedback from operators using Table 3.5.3 below.

Table 3.5.3 - Time measured in seconds when using various test modes

\begin{tabular}{|c|c|c|c|c|c|c|c|}
\hline \multirow{2}{*}{ Test Mode } & \multicolumn{3}{|c|}{$\begin{array}{l}\text { Time completed } \\
\text { (s) }\end{array}$} & \multirow{2}{*}{$\begin{array}{l}\text { Average time } \\
\text { completed in } \\
\text { (s) }\end{array}$} & \multirow{2}{*}{$\begin{array}{c}\text { Total time } \\
\text { completed in } \\
\text { (s) }\end{array}$} & \multirow{2}{*}{$\begin{array}{c}\# \text { of } \\
\text { successful } \\
\text { test } \\
\text { completes }\end{array}$} & \multirow[t]{2}{*}{$\begin{array}{c}\text { Fault Conditions } \\
\text { Feedback }\end{array}$} \\
\hline & $\begin{array}{l}\text { 1st } \\
\text { Run }\end{array}$ & $\begin{array}{l}\text { 2nd } \\
\text { Run }\end{array}$ & $\begin{array}{l}\text { 3rd } \\
\text { Run }\end{array}$ & & & & \\
\hline 3 -Axis & & & & & & & \\
\hline Angular & & & & & & & \\
\hline API & & & & & & & \\
\hline
\end{tabular}


Operators continue on the next object until all three objects have been picked up and dropped to the target location. Operators repeat this for the next test mode until all three test modes have been completed or abandoned.

\subsection{Summary}

The LF method presented in this chapter allows almost anybody to safely control a distant $\mathrm{MICO}^{2}$ arm by physically manipulating another $\mathrm{MICO}^{2}$ arm either with his/her hands or with a standard joystick controller. With the implementation of the LF method, robot arm operators are able to train themselves on how to manipulate effectively so that the way human-robot interaction controls are more interesting. Although operators are able to move the arm freely by manually pushing or pulling on some link, there are some limitations that operators should be aware of when controlling the arm. Similar to a human arm, each actuator or each finger of the $\mathrm{MICO}^{2}$ arm has its own range limit. If operators try to send a command that goes beyond the acceptable values, the arm will stop moving.

Generally, there are various techniques that can be used to control the movements of robot arms. Each technique has its own way to trigger motions of robot arms; each technique has both advantages and disadvantages. Overall, these techniques provide humans with many different approaches to remotely control robot arms. By applying the LF method, remote robot manipulation system allows operators to control one arm while indirectly viewing another remote arm and use the remote arm to potentially dispose of pipe bombs while operating the system from a safe distance. 


\section{Chapter 4}

\section{Experiments and Results}

\subsection{Introduction}

Performance tests were conducted using the test apparatus and associated test procedures to determine the relative efficacy of the manipulator grasping and dropping capability of a remotely teleoperated robot arm across a statistically significant number of test sessions involving grasping, picking up and dropping objects using various test methods, as described in section 3.5.

This chapter analyses experimental results from three test methods obtained from various levels of robotic experiences by users when performing a number of grasping, picking up and dropping tests in a controlled environment equipped with twin Kinova robot arms. In this chapter, we will also discuss in more detail how different test methods can be used to measurably assess the manipulator grasping and dropping capability of a remotely teleoperated robot arm followed by a demonstration of the efficacy of the LF method in relation to other control strategies.

Timings to complete tasks are recorded as an average time to perform each task within each test mode that can produce different levels of completeness. In this way, the final results are a compendium of average completion times and can be used to assess the relative effectiveness of various ways of controlling a robot arm. 


\subsection{Experimental Results}

The following subsections describe the associated test methods and final experimental results obtained.

\subsubsection{Experimental Results for Controlling in Angular Mode}

As described in section 3.2, robot arm operators can manually control each joint by using a joystick and rotating it with a specific angle positively or negatively when the robot arm is set to Angular mode. The arm's collision avoidance mode is automatically disabled during angular control. Therefore, controlling the robot arm in this mode requires additional attention from the operator to avoid any possible collisions between the end effector and the rest of the arm. Similar to a human arm, each joint (or finger) of the robot arm does have its own range limit that operators are allowed to control. The details of software position limitations of actuators and fingers can be found in Appendix C.

Since the $\mathrm{MICO}^{2}$ arm allows each joint to be controlled individually, operators might experience some difficulties trying to move the arm forward or backward as a unit. This could be even harder when operators have to deal with complex manipulations without being able to directly view the arm--being forced to interpret the position of the arm through a quad display as described in detail in Appendix B. Figure 4.2.1A illustrates a process consisting of several steps for combining multiple joints to create distinct poses required to bring the end effector of the robot arm lower and/or closer to target objects. This process is challenging for operators with limited experience in remotely manipulating the robot arm. 


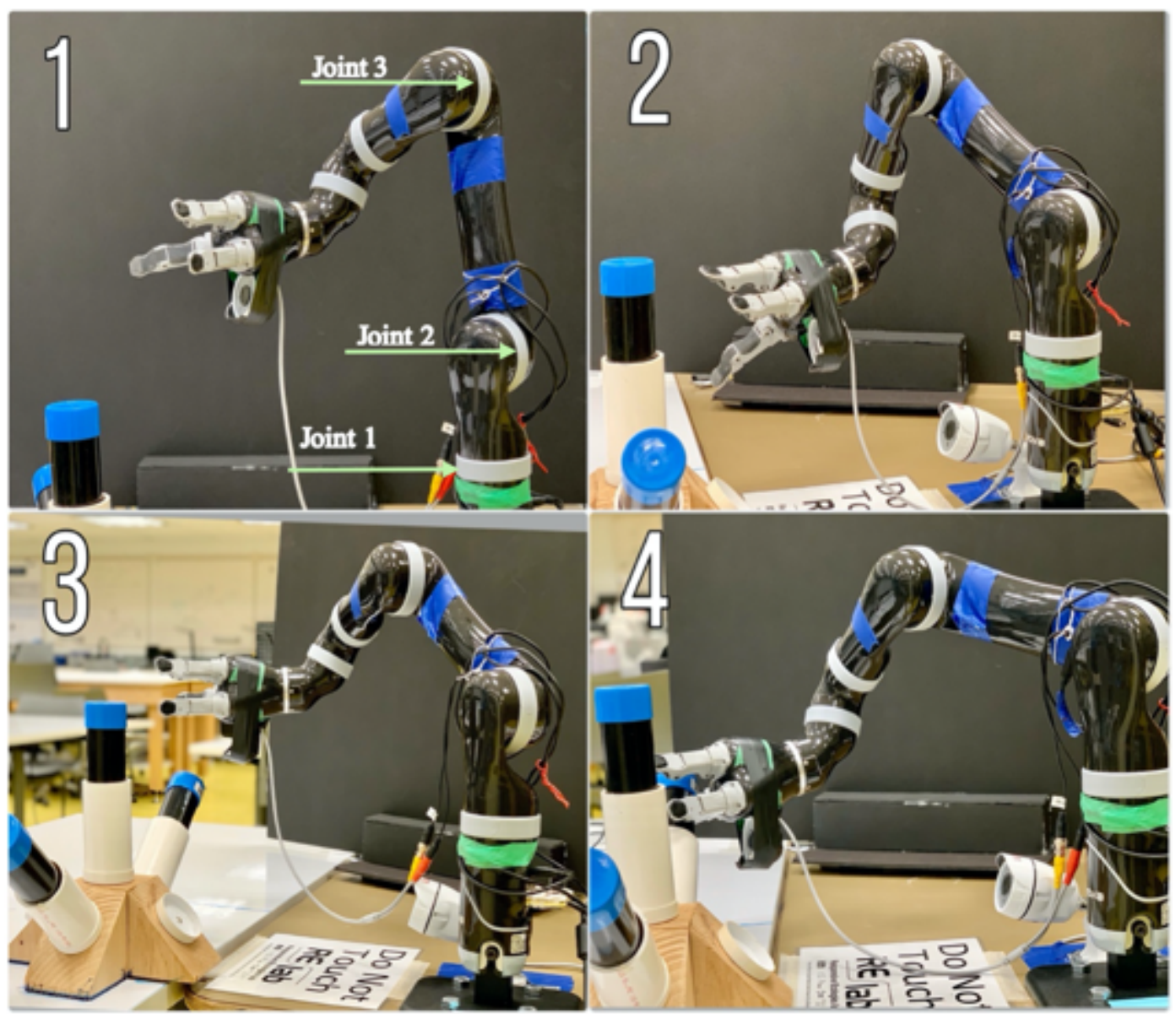

Figure 4.2.1A - Kinova $\mathrm{MICO}^{2}$ arm in several distinct poses

In this test mode, a few operators were not able to complete the tests successfully due to the following:

- insufficient practice to become familiar enough with the control strategy

- extra time needed to complete each task

- failure to drop an object in the required location

In addition, one operator was not able to finish the assigned task due to hardware failure during the operation. The overall test results were captured and summarized in detail in Table 4.2.1 below. 
Table 4.2.1 - Overall average and total time measured in seconds in Angular mode

\begin{tabular}{|c|c|c|c|c|c|c|c|}
\hline \multirow[t]{2}{*}{$\begin{array}{c}\text { Operator } \\
\#\end{array}$} & \multicolumn{3}{|c|}{$\begin{array}{c}\text { Time completed } \\
\text { (s) }\end{array}$} & \multirow{2}{*}{$\begin{array}{l}\text { Average time } \\
\text { completed in } \\
\text { (s) }\end{array}$} & \multirow{2}{*}{$\begin{array}{l}\text { Total time } \\
\text { completed in } \\
\text { (s) }\end{array}$} & \multirow{2}{*}{$\begin{array}{c}\text { \# of } \\
\text { successful } \\
\text { test } \\
\text { completes }\end{array}$} & \multirow[t]{2}{*}{$\begin{array}{c}\text { Fault conditions/ } \\
\text { Feedback }\end{array}$} \\
\hline & $\begin{array}{c}\text { 1st } \\
\text { Run }\end{array}$ & $\begin{array}{l}\text { 2nd } \\
\text { Run }\end{array}$ & $\begin{array}{l}\text { 3rd } \\
\text { Run }\end{array}$ & & & & \\
\hline 1 & 269.00 & 203.00 & 161.00 & 211.00 & 633.00 & 3 & N/A \\
\hline 2 & 109.00 & 123.00 & 69.00 & 100.33 & 301.00 & 3 & $\mathrm{~N} / \mathrm{A}$ \\
\hline 3 & 54.00 & 56.00 & 68.00 & 59.33 & 178.00 & 3 & N/A \\
\hline 4 & 73.00 & 127.00 & 115.00 & 105.00 & 315.00 & 3 & N/A \\
\hline 5 & 118.00 & 134.00 & 0.00 & 126.00 & 252.00 & 2 & N/A \\
\hline 6 & 62.00 & 270.00 & 122.00 & 151.33 & 454.00 & 3 & $\mathrm{~N} / \mathrm{A}$ \\
\hline 7 & 33.00 & 108.00 & 54.00 & 65.00 & 195.00 & 3 & $\mathrm{~N} / \mathrm{A}$ \\
\hline 8 & 29.00 & 36.00 & 104.00 & 56.33 & 169.00 & 3 & $\mathrm{~N} / \mathrm{A}$ \\
\hline 9 & 182.00 & 106.00 & 118.00 & 135.33 & 406.00 & 3 & $\mathrm{~N} / \mathrm{A}$ \\
\hline 10 & 77.00 & 119.00 & 117.00 & 104.33 & 313.00 & 3 & N/A \\
\hline 11 & 27.00 & 63.00 & 105.00 & 65.00 & 195.00 & 3 & N/A \\
\hline 12 & 34.00 & 56.00 & 52.00 & 47.33 & 142.00 & 3 & N/A \\
\hline 13 & 61.00 & 34.00 & 0.00 & 47.50 & 95.00 & 2 & Hardware Failed \\
\hline 14 & 0.00 & 0.00 & 0.00 & 0.00 & 0.00 & 0 & Gave up \\
\hline 15 & 0.00 & 0.00 & 0.00 & 0.00 & 0.00 & 0 & Gave up \\
\hline 16 & 0.00 & 0.00 & 0.00 & 0.00 & 0.00 & 0 & Gave up \\
\hline 17 & 247.00 & 378.00 & 386.00 & 337.00 & 1011.00 & 3 & $\mathrm{~N} / \mathrm{A}$ \\
\hline 18 & 0.00 & 0.00 & 0.00 & 0.00 & 0.00 & 0 & Gave up \\
\hline 19 & 59.00 & 119.00 & 81.00 & 86.33 & 259.00 & 3 & $\mathrm{~N} / \mathrm{A}$ \\
\hline 20 & 48.00 & 146.00 & 65.00 & 86.33 & 259.00 & 3 & N/A \\
\hline 21 & 59.00 & 68.00 & 203.00 & 110.00 & 330.00 & 3 & N/A \\
\hline 22 & 118.00 & 115.00 & 117.00 & 116.67 & 350.00 & 3 & N/A \\
\hline 23 & 121.00 & 195.00 & 167.00 & 161.00 & 483.00 & 3 & N/A \\
\hline 24 & 175.00 & 212.00 & 141.00 & 176.00 & 528.00 & 3 & N/A \\
\hline 25 & 86.00 & 235.00 & 164.00 & 161.67 & 485.00 & 3 & N/A \\
\hline 26 & 112.00 & 65.00 & 0.00 & 88.50 & 177.00 & 2 & N/A \\
\hline 27 & 116.00 & 226.00 & 143.00 & 161.67 & 485.00 & 3 & N/A \\
\hline 28 & 96.00 & 167.00 & 100.00 & 121.00 & 363.00 & 3 & N/A \\
\hline 29 & 187.00 & 111.00 & 123.00 & 140.33 & 421.00 & 3 & N/A \\
\hline 30 & 164.00 & 178.00 & 124.00 & 155.33 & 466.00 & 3 & N/A \\
\hline
\end{tabular}




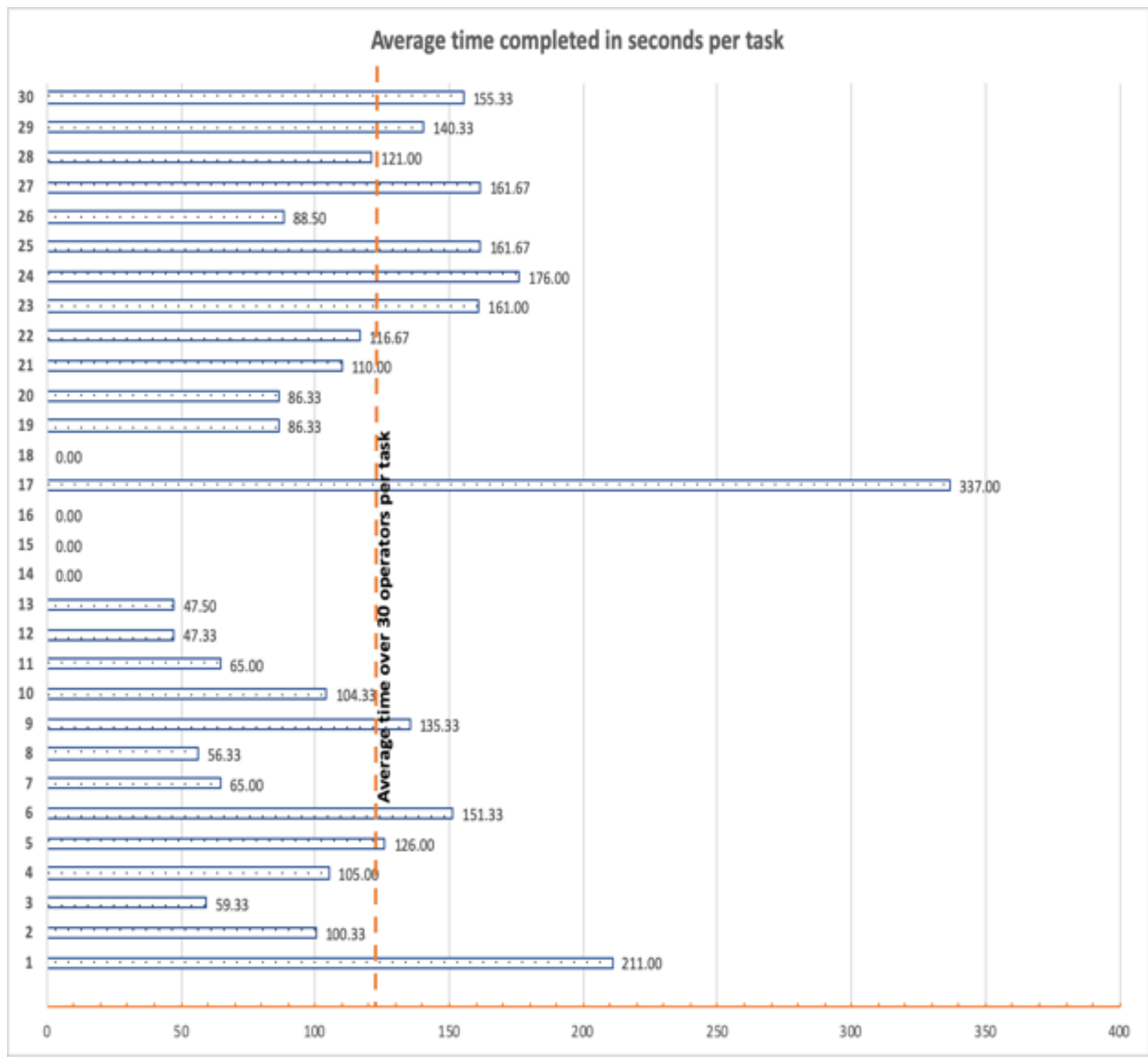

Figure 4.2.1B - Average time measured in seconds per task in Angular mode

In Figure 4.2.1B above, each bar along the $\mathrm{X}$ axis represents each operator's tested average speed per task complete, measured in seconds. The orange dotted line illustrates an overall average speed for 30 tested operators which was approximately $122.14 \mathrm{~s}$ while the longest trial took around 337.00 s to complete the same task. 0.00 means that operators were not able to complete their tests. 


\subsubsection{Experimental Results for Controlling in 3-Axis Mode}

Kinova robot arms can also be controlled by the end effector position when using the joystick in Cartesian mode, either in 3-Axis or in 2-Axis operation mode. In this work, we are only interested in 3-Axis mode. The operating principles are much simpler and easier to use as compared with controlling an arm in Angular mode. In 3-Axis mode, operators can perform many basic movements by controlling the end effector's translations (position variations) with respect to its base and the rotations (orientation variations) around the end effector's reference point. This 3Axis mode, in contrast to Angular mode, allows operators to quickly gain control of any positions of the end effector in space while keeping it parallel with its base - allowing operators a more flexible way to interpret the current position of the remote arm through a quad display. While in Translation mode, as depicted in section 3.3, any translation X allows operators to move the end effector to left or right whereas any translation $\mathrm{Y}$ lets operators move the end effector forward or backward or move the end effector up or down by simply making a change to translation $\mathrm{Z}$. Similarly, the control is also straightforward when switching to Wrist mode.

However, the robot arm, in Cartesian mode, might lose one or more degrees of freedom due to singularity auto-avoidance behaviour (see Appendix D for details). This means the robot arm is not able to move in one direction or the other; therefore, any input commands generated by operators will be corrected somewhat to avoid a singularity. When this situation occurs, operators are not able to move the end effector in Translation mode anymore.

There is no need to switch the control of each joint manually as compared with operating the arm in Angular mode, operators are able to maneuver the arm in various poses through space using a 4 quadrant display as illustrated in the following photos numbered from 1 through 4 of Figure 4.2.2A. When the robot receives an input command, it computes a straight line from its end 
effector's current position to the next given trajectory point and performs a translation, assuming that the desired trajectory point is reachable.

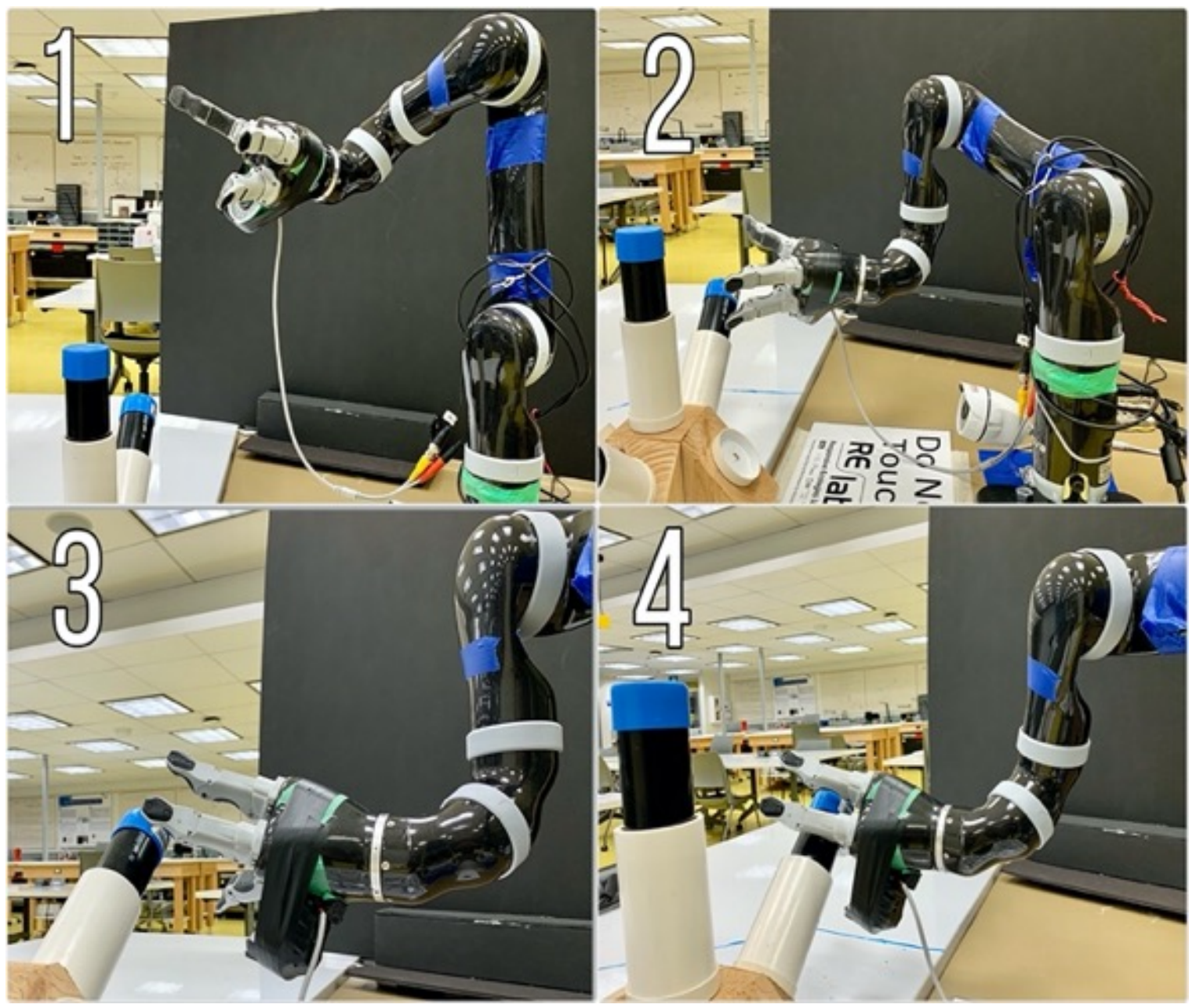

Figure 4.2.2A - Various poses transformed when in 3-Axis mode

As described in chapter 3, each operator is randomly assigned to a test mode that requires the transfer of three objects from an initial location to a designed final location. The core intention of random task assignment is to ensure that operators did not pick up unintentional skills through the use of the robot arm's controls in a previous control mode. Our intention is to sure that the collected data over all operators remains unbiased. 
Table 4.2.2 - Overall average and total time measured in seconds in 3-Axis mode

\begin{tabular}{|c|c|c|c|c|c|c|c|}
\hline \multirow[t]{2}{*}{$\begin{array}{c}\text { Operator } \\
\#\end{array}$} & \multicolumn{3}{|c|}{$\begin{array}{l}\text { Time completed } \\
\text { (s) }\end{array}$} & \multirow{2}{*}{$\begin{array}{l}\text { Average time } \\
\text { completed in } \\
\text { (s) }\end{array}$} & \multirow{2}{*}{$\begin{array}{l}\text { Total time } \\
\text { completed in } \\
\text { (s) }\end{array}$} & \multirow{2}{*}{$\begin{array}{c}\# \text { of } \\
\text { successful } \\
\text { test } \\
\text { completes }\end{array}$} & \multirow[t]{2}{*}{$\begin{array}{c}\text { Fault conditions/ } \\
\text { Feedback }\end{array}$} \\
\hline & $\begin{array}{c}\text { 1st } \\
\text { Run }\end{array}$ & $\begin{array}{l}\text { 2nd } \\
\text { Run }\end{array}$ & $\begin{array}{l}\text { 3rd } \\
\text { Run }\end{array}$ & & & & \\
\hline 1 & 142.00 & 267.00 & 218.00 & 209.00 & 627.00 & 3 & N/A \\
\hline 2 & 52.00 & 80.00 & 130.00 & 87.33 & 262.00 & 3 & N/A \\
\hline 3 & 68.00 & 100.00 & 85.00 & 84.33 & 253.00 & 3 & N/A \\
\hline 4 & 79.00 & 106.00 & 127.00 & 104.00 & 312.00 & 3 & N/A \\
\hline 5 & 39.00 & 115.00 & 100.00 & 84.67 & 254.00 & 3 & N/A \\
\hline 6 & 66.00 & 158.00 & 150.00 & 124.67 & 374.00 & 3 & N/A \\
\hline 7 & 25.00 & 97.00 & 62.00 & 61.33 & 184.00 & 3 & N/A \\
\hline 8 & 27.00 & 82.00 & 59.00 & 56.00 & 168.00 & 3 & $\mathrm{~N} / \mathrm{A}$ \\
\hline 9 & 45.00 & 75.00 & 171.00 & 97.00 & 291.00 & 3 & $\mathrm{~N} / \mathrm{A}$ \\
\hline 10 & 84.00 & 159.00 & 116.00 & 119.67 & 359.00 & 3 & N/A \\
\hline 11 & 29.00 & 77.00 & 48.00 & 51.33 & 154.00 & 3 & N/A \\
\hline 12 & 43.00 & 105.00 & 97.00 & 81.67 & 245.00 & 3 & N/A \\
\hline 13 & 59.00 & 81.00 & 77.00 & 72.33 & 217.00 & 3 & N/A \\
\hline 14 & 74.00 & 129.00 & 120.00 & 107.67 & 323.00 & 3 & N/A \\
\hline 15 & 41.00 & 190.00 & 125.00 & 118.67 & 356.00 & 3 & N/A \\
\hline 16 & 198.00 & 310.00 & 326.00 & 278.00 & 834.00 & 3 & N/A \\
\hline 17 & 76.00 & 135.00 & 162.00 & 124.33 & 373.00 & 3 & N/A \\
\hline 18 & 56.00 & 125.00 & 101.00 & 94.00 & 282.00 & 3 & N/A \\
\hline 19 & 66.00 & 124.00 & 226.00 & 138.67 & 416.00 & 3 & N/A \\
\hline 20 & 82.00 & 187.00 & 178.00 & 149.00 & 447.00 & 3 & N/A \\
\hline 21 & 57.00 & 142.00 & 134.00 & 111.00 & 333.00 & 3 & N/A \\
\hline 22 & 118.00 & 117.00 & 116.00 & 117.00 & 351.00 & 3 & N/A \\
\hline 23 & 118.00 & 115.00 & 117.00 & 116.67 & 350.00 & 3 & N/A \\
\hline 24 & 89.00 & 187.00 & 239.00 & 171.67 & 515.00 & 3 & N/A \\
\hline 25 & 108.00 & 58.00 & 72.00 & 79.33 & 238.00 & 3 & N/A \\
\hline 26 & 59.00 & 81.00 & 77.00 & 72.33 & 217.00 & 3 & N/A \\
\hline 27 & 35.00 & 96.00 & 67.00 & 66.00 & 198.00 & 3 & N/A \\
\hline 28 & 126.00 & 89.00 & 74.00 & 96.33 & 289.00 & 3 & N/A \\
\hline 29 & 122.00 & 111.00 & 88.00 & 107.00 & 321.00 & 3 & N/A \\
\hline 30 & 104.00 & 108.00 & 84.00 & 98.67 & 296.00 & 3 & N/A \\
\hline
\end{tabular}

When using 3-Axis mode, all operators were able to successfully accomplish the same set of tasks with an improved average time recorded in Table 4.2.2 above. By comparing the dotted line against the actual average times needed in the figure below, one can notice a slightly better performance 
acquired by the same operators. Overall, the average speed is close to $109.32 \mathrm{~s}$ whereas the longest trial took 278.00 s to complete the equivalent task.

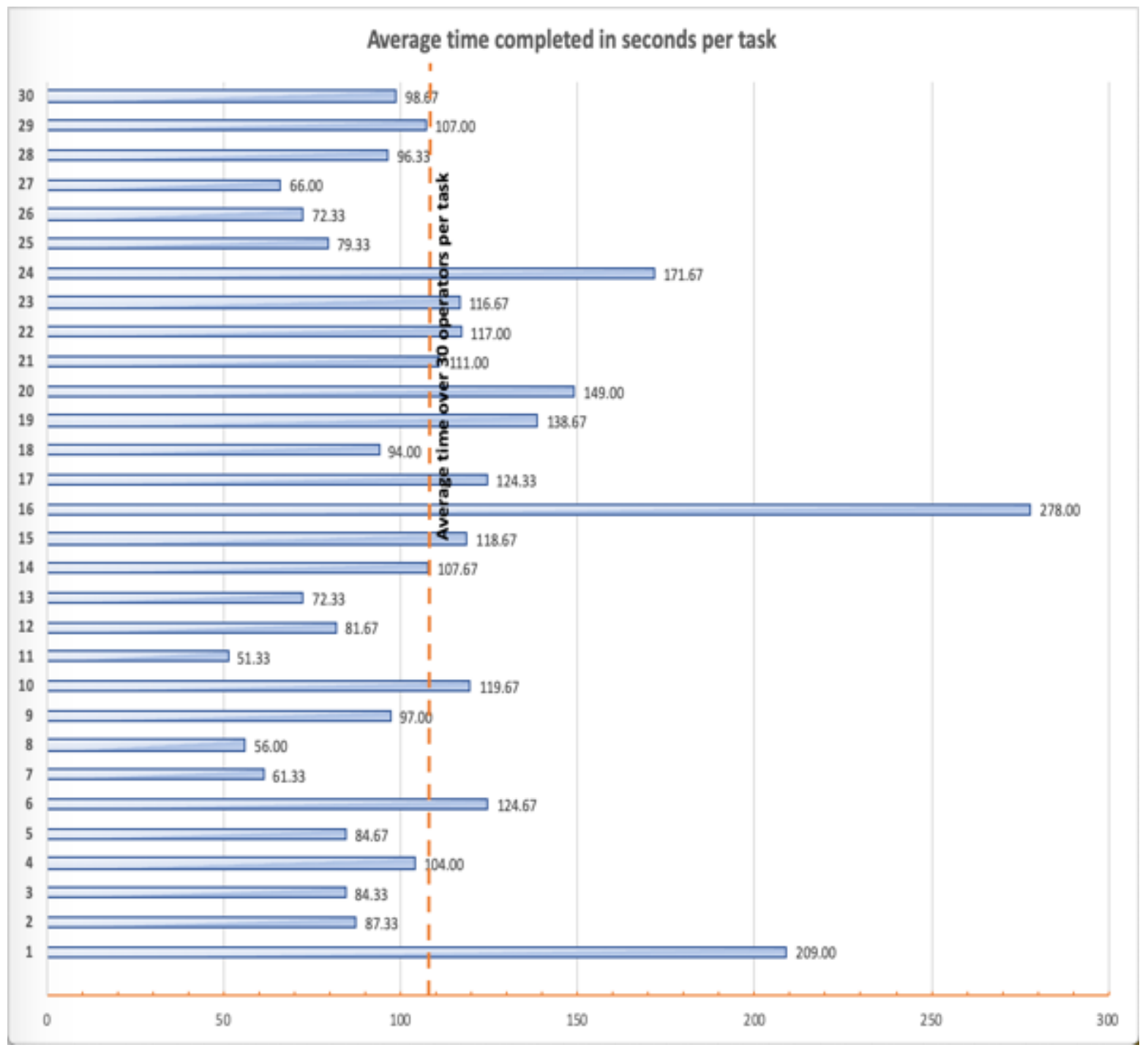

Figure 4.2.2B - Average time measured in seconds per task in 3-Axis mode

In general, from Figure 4.2.2B above, each bar plotted horizontally represents a smaller average time needed to complete the equivalent task when compared with results obtained from the Angular mode. For this reason, we believe that choosing an effective control strategy is a key factor determining success when dealing with remote complex manipulations. 


\subsubsection{Experimental Results for the LF Method}

Some operators were clearly more familiar with operating devices using joysticks than others which gave them an advantage because at least the control paradigm was familiar to them. Without the need for a joystick, the LF method allows operators with different levels of experience (including none) to control a remote arm by physically manipulating the movement of another local arm. The results of our work demonstrate three important things:

- It takes less time for operators to use the robot arm employing the LF technique

- Higher success rate when dealing with various remote complex manipulations

- More flexible for operators as they are required to interpret various difficult poses of the remote arm via a fixed quad display

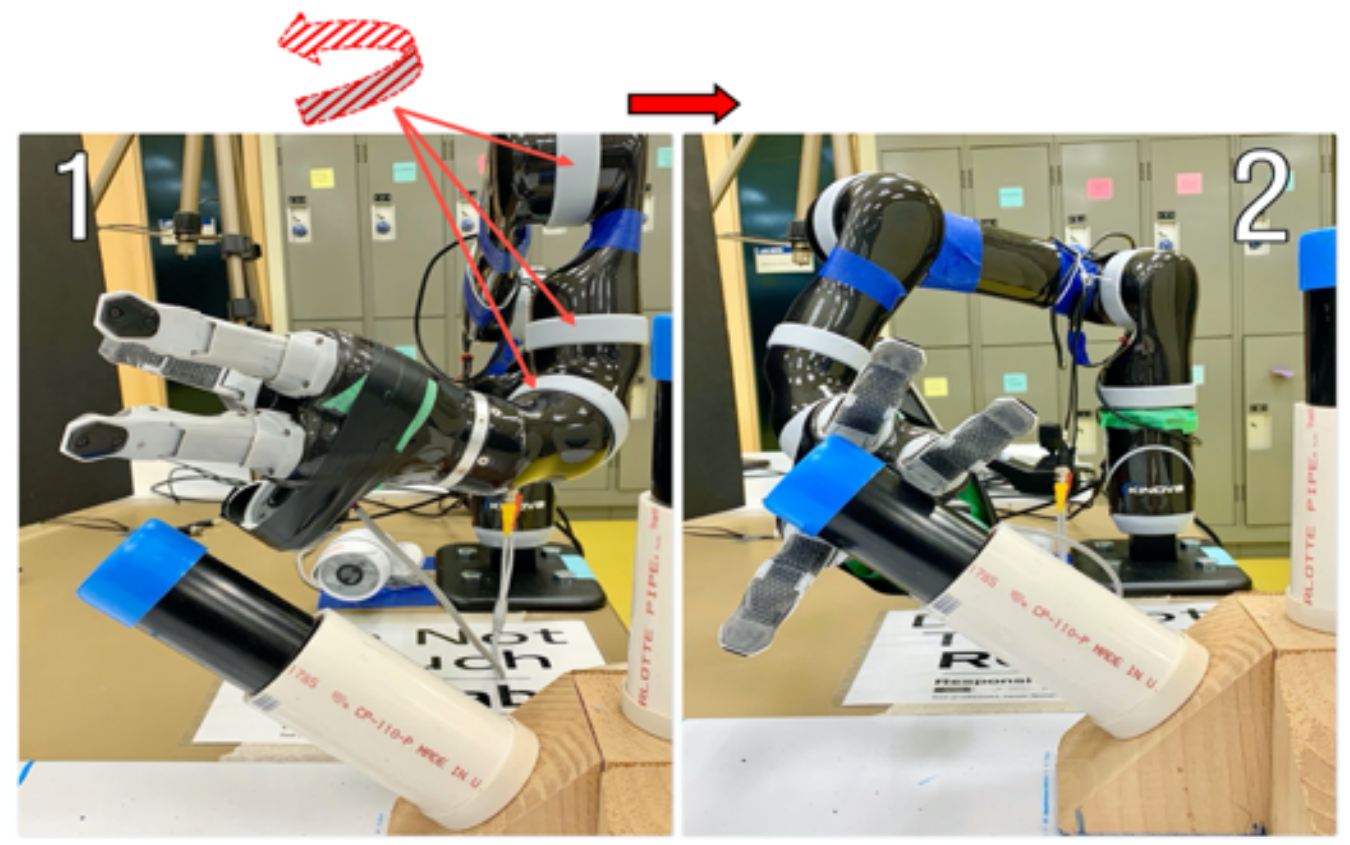

Figure 4.2.3A - Robot arms synchronized in LF mode by physically pushing or pulling on some link on the operator arm

A closer examination of the scenario as illustrated in Figure 4.2.3A above reveals that directly pushing or pulling on some actuators of the local arm when attempting to align the end effector of 
the remote arm with the target object is almost effortless. The LF method, is demonstrated to be one of the simplest and flexible control techniques we believe because operators can not only replicate robot arm positions and movements between the two robot arms, but can also effectively interpret different situations of the remote arm via a fixed quad display and their ability to view the local leader robot arm.

Table 4.2.3 - Overall average and total time measured in seconds in the LF mode

\begin{tabular}{|c|c|c|c|c|c|c|c|}
\hline $\begin{array}{c}\text { Operator } \\
\#\end{array}$ & \multicolumn{2}{|c|}{ Time completed } & $\begin{array}{c}\text { Average time } \\
\text { completed in } \\
\text { (s) }\end{array}$ & $\begin{array}{c}\text { Total time } \\
\text { completed in } \\
\text { (s) }\end{array}$ & $\begin{array}{c}\text { \# of } \\
\text { successful } \\
\text { test } \\
\text { completes }\end{array}$ & $\begin{array}{c}\text { Fault conditions/ } \\
\text { Feedback }\end{array}$ \\
\cline { 2 - 5 } Run & $\begin{array}{c}\text { 2nd } \\
\text { Run }\end{array}$ & $\begin{array}{c}\text { 3rd } \\
\text { Run }\end{array}$ & & & \\
\hline 1 & 145.00 & 136.00 & 121.00 & 134.00 & 402.00 & 3 & N/A \\
\hline 2 & 96.00 & 44.00 & 73.00 & 71.00 & 213.00 & 3 & N/A \\
\hline 3 & 81.00 & 61.00 & 52.00 & 64.67 & 194.00 & 3 & N/A \\
\hline 4 & 42.00 & 89.00 & 74.00 & 68.33 & 205.00 & 3 & N/A \\
\hline 5 & 53.00 & 81.00 & 82.00 & 72.00 & 216.00 & 3 & N/A \\
\hline 6 & 59.00 & 93.00 & 120.00 & 90.67 & 272.00 & 3 & N/A \\
\hline 7 & 34.00 & 62.00 & 81.00 & 59.00 & 177.00 & 3 & N/A \\
\hline 8 & 32.00 & 67.00 & 62.00 & 53.67 & 161.00 & 3 & N/A \\
\hline 9 & 34.00 & 82.00 & 47.00 & 54.33 & 163.00 & 3 & N/A \\
\hline 10 & 127.00 & 118.00 & 93.00 & 112.67 & 338.00 & 3 & N/A \\
\hline 11 & 37.00 & 51.00 & 40.00 & 42.67 & 128.00 & 3 & N/A \\
\hline 12 & 32.00 & 55.00 & 66.00 & 51.00 & 153.00 & 3 & N/A \\
\hline 13 & 49.00 & 48.00 & 103.00 & 66.67 & 200.00 & 3 & N/A \\
\hline 14 & 122.00 & 53.00 & 73.00 & 82.67 & 248.00 & 3 & N/A \\
\hline 15 & 58.00 & 113.00 & 84.00 & 85.00 & 255.00 & 3 & N/A \\
\hline 16 & 69.00 & 109.00 & 141.00 & 106.33 & 319.00 & 3 & N/A \\
\hline 17 & 80.00 & 70.00 & 171.00 & 107.00 & 321.00 & 3 & N/A \\
\hline 18 & 180.00 & 174.00 & 151.00 & 168.33 & 505.00 & 3 & N/A \\
\hline 19 & 107.00 & 68.00 & 57.00 & 77.33 & 232.00 & 3 & N/A \\
\hline 20 & 63.00 & 62.00 & 62.00 & 62.33 & 187.00 & 3 & N/A \\
\hline 21 & 43.00 & 99.00 & 73.00 & 71.67 & 215.00 & 3 & N/A \\
\hline 22 & 113.00 & 72.00 & 111.00 & 98.67 & 296.00 & 3 & N/A \\
\hline 23 & 107.00 & 83.00 & 93.00 & 94.33 & 283.00 & 3 & N/A \\
\hline 24 & 46.00 & 52.00 & 73.00 & 57.00 & 171.00 & 3 & N/A \\
\hline 25 & 73.00 & 81.00 & 83.00 & 79.00 & 237.00 & 3 & N/A \\
\hline 26 & 28.00 & 42.00 & 36.00 & 35.33 & 106.00 & 3 & N/A \\
\hline 27 & 52.00 & 72.00 & 62.00 & 62.00 & 186.00 & 3 & N/A \\
\hline 28 & 52.00 & 72.00 & 62.00 & 62.00 & 186.00 & 3 & N/A \\
\hline 29 & 41.00 & 89.00 & 53.00 & 61.00 & 183.00 & 3 & N/A \\
\hline 30 & 77.00 & 87.00 & 88.00 & 84.00 & 252.00 & 3 & N/A \\
\hline & & & & & & & 3 \\
\hline
\end{tabular}


Noticeably, an improved average time obtained from Table 4.2.3 above is considerable evidence that the LF method overcame the other two test modes regarding time and number of successful tests completed. Within a short period of time needed for self-training, operators can rapidly acquaint themselves with robot control techniques in a remote testing environment through a quad display. Nevertheless, there is a minor drawback of our proposed method due to a very small timedelay between each movement. This is interpreted as the data input of each joint and each finger of a local arm needs to be scaled respectively prior to being translated into new sets of $\mathrm{x}, \mathrm{y}, \mathrm{z}$ coordinates of another remote arm required to mimic the current pose. Though this is negligible, operators are expected to move each joint quickly but accurately in order to achieve the best time.

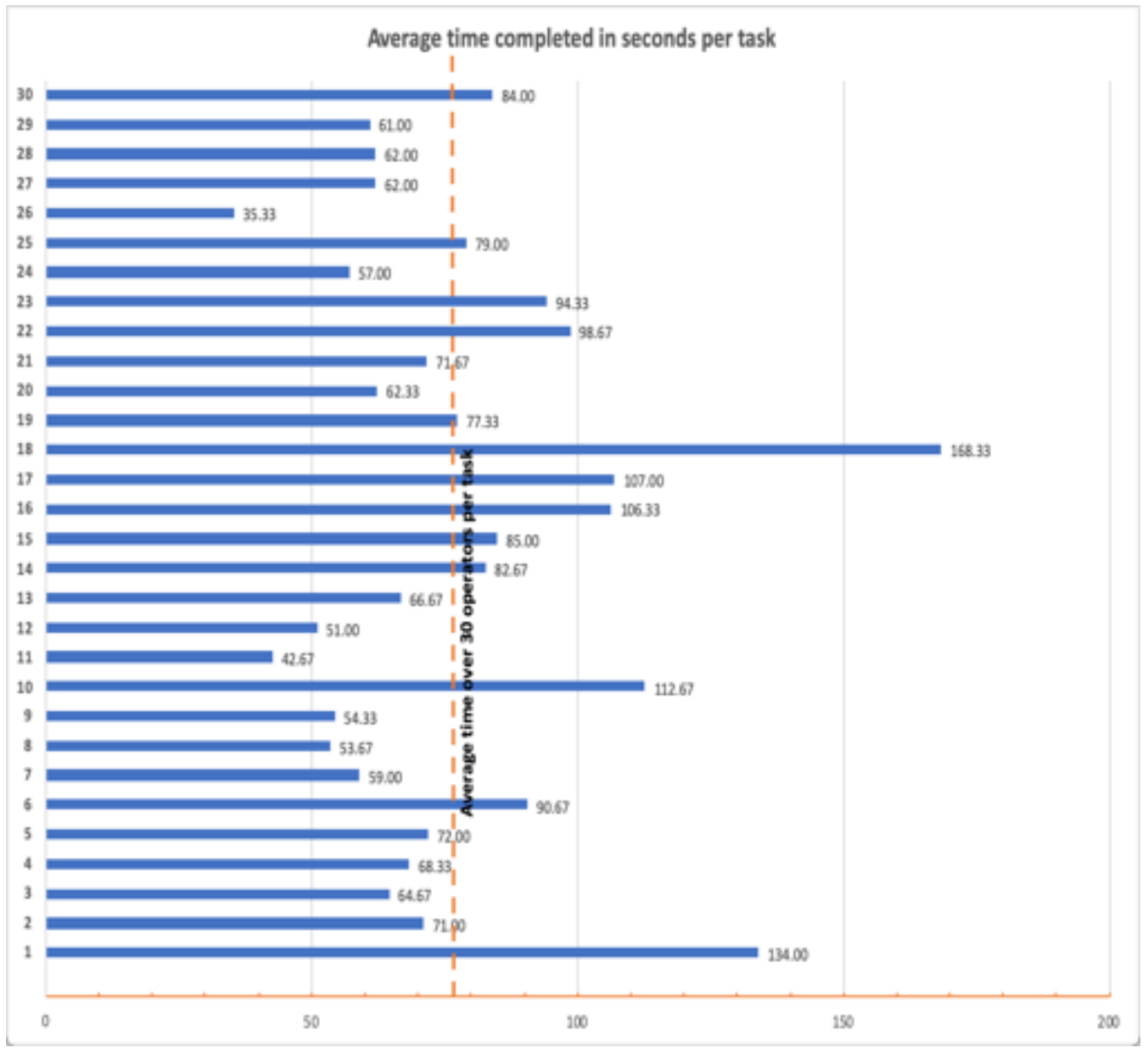

Figure 4.2.3B - Average time measured in seconds per task in the LF method 
With an average of $77.82 \mathrm{~s}$, the bar chart shown in Figure 4.2.3B showed that the overall average time needed to complete the same tasks is much quicker when comparing to the results obtained from Angular mode (122.14s as depicted in Figure 4.2.1B) and 3-Axis mode (109.32s as depicted in Figure 4.2.2B). Consequently, this is very vital as final data comparison can be considered as baseline evaluations when remote manipulation becomes a primary consideration.

\subsection{The Challenge}

The documentation of various Response Robot Evaluation Exercises developed and run by NIST [145] over the years which includes detailed descriptions of many different test methods that have been developed to address the needs of emergency response operations in order to objectively measure various aspects of response robots - including arms. These standard test methods can be used to provide practice tasks and capability objectives that exercise respective robotic control strategies. One of them is labelled as "New Test Method for Evaluating Ground Response Robot Dexterity: Grasp Load, Traverse, and Place" which has been proposed as a part of a suite of robotic manipulation test methods and can be used to evaluate a remotely teleoperated robots' capability of conducting a Grasp Load, Traverse, and Place task [146]. Not only can the proposed test method be used to both demonstrate control and measure its efficacy in relation to other test methods, but it can also be helpful for diminishing operator risk when approaching suspicious devices. The robotic manipulator is to be evaluated on the effectiveness of grasping and dropping objects in repeatable ways to facilitate direct comparisons among different control strategies. The standard metric used during evaluation exercises is an average task rate which provides a relative indication of performance of different control strategies. 
In order to achieve remote manipulation via a robot arm, we applied several common ways of remotely controlling robot arms including:

- Joystick control in both 3-Axis and Angular modes

- Software control and API control when using a personal computer

Performance data collections were conducted using the same test apparatus and associated test procedure to capture the capability of remote operator performance over a significant number of repetitions. Timing measures are reported as an average time to perform each repetition within a test method which can produce varying levels of completeness. As a consequence, final results can be considered as baseline evaluations for determining the best way of deploying robot arms for a remote manipulation task.

\subsection{Analysis}

Overall, operators with different levels of experience were able to establish their proficiency prior to completing the required number of repetitions for all three test methods. Each of these test methods has its own level of difficulty depending on the operators' abilities. Despite the fact that operators had different levels of skill and were able to complete their tests, remote manipulation is difficult when information about the task is only available through a quad display.

Most robotic arms have been designed to be fully articulated. They usually have up to 6 degrees of freedom, use rotary joints to access the peripherals, and are able to perform various intricate and dexterous movements as a human arm does. Thus, there is a lot of work associated with a robotic arm that needs to bend and move - for which actuators are needed. Certainly, operators can simply use the joystick provided to manage each actuator one by one when the arm is in 
Angular mode, but operators might encounter some challenges with achieving an arm-like rotation movement in both directions. Or maybe some operators feel more comfortable with operating as a set of multiple actuators in either Translation mode or Wrist mode when the control mode is in 3Axis (Cartesian). However, the robot arm's usable workspace is slightly smaller when it's in Cartesian mode due to singularities, self-collisions, and protection zones (defined near the base connectors of the robot arm) automatic avoidance algorithms embedded in the kinematics of the arm. When the robot arm reaches any of these situations, operators are not able to continue moving in the same direction but are able to divert to movement in unrestricted spaces.

We have demonstrated that the LF control method is a very flexible, easy to learn and accurate control technique as we discovered by testing and measuring the manipulator grasping and dropping capability for the same relevant object with various case scenarios as shown in Figure 4.4.1 below when compared to the other common control techniques used in the same testing framework.

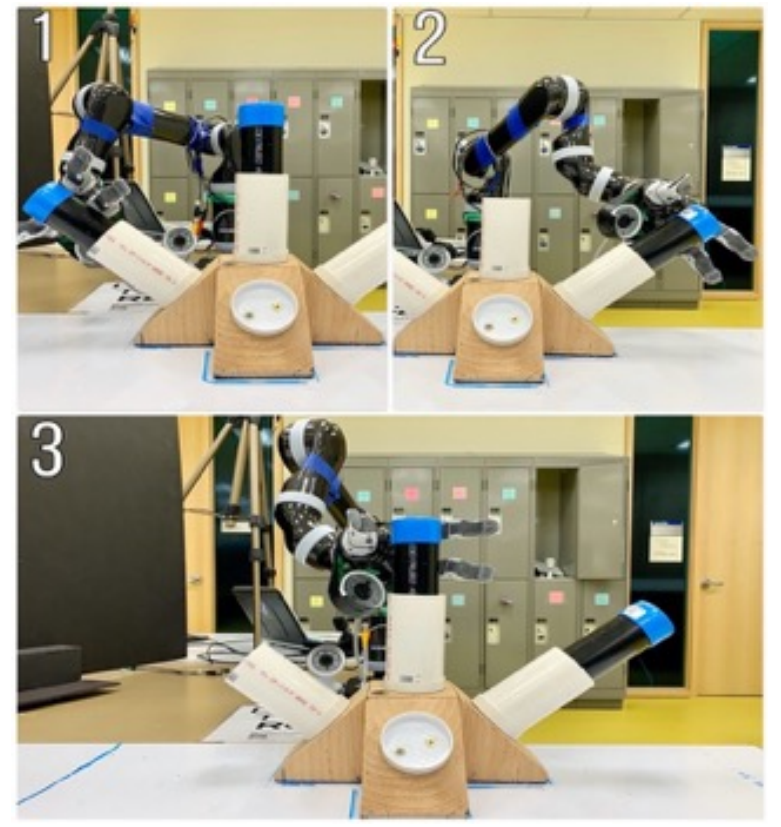

Figure 4.4.1 - Simulated pipe bombs used during experiments with various case scenarios 
Operational scenarios can be simulated with the test methods we used, providing an indication of robot capabilities while using different control strategies. Each operational task was tested in repeatable ways for the purposes of establishing statistical significance and providing measurable performance differences among several control strategies. The overall average time for each run and the average total time needed in seconds were recorded and are illustrated in the following charts (Figure 4.4.2).

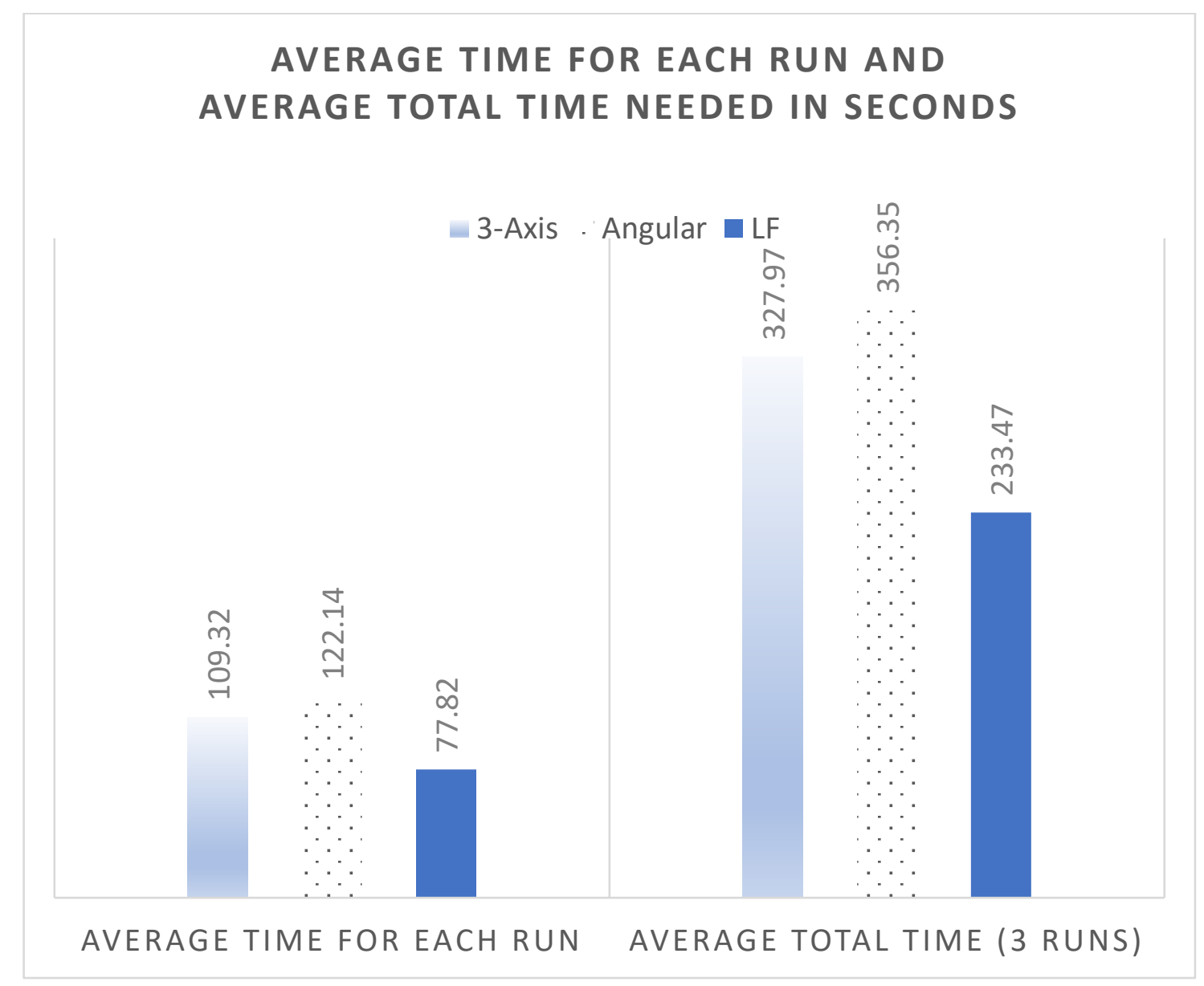

Figure 4.4.2 - Average time per task and average total time needed

In discussing these results, a graphical bar chart generated from the overall performance based on 30 tested operators, as shown in Figure 4.4.3 below, clearly indicates that all operators were able to successfully complete three runs for each test method. Each bar along the $\mathrm{X}$ axis represents the 
number of successful tests completed per test mode. However, a lower success rate was recorded when using the Angular method (orange bars) in comparison to the other two test methods. There are a few operators $(14,15,16$ and 18$)$ were not able to perform the Agular method and ended up with giving up their tests.

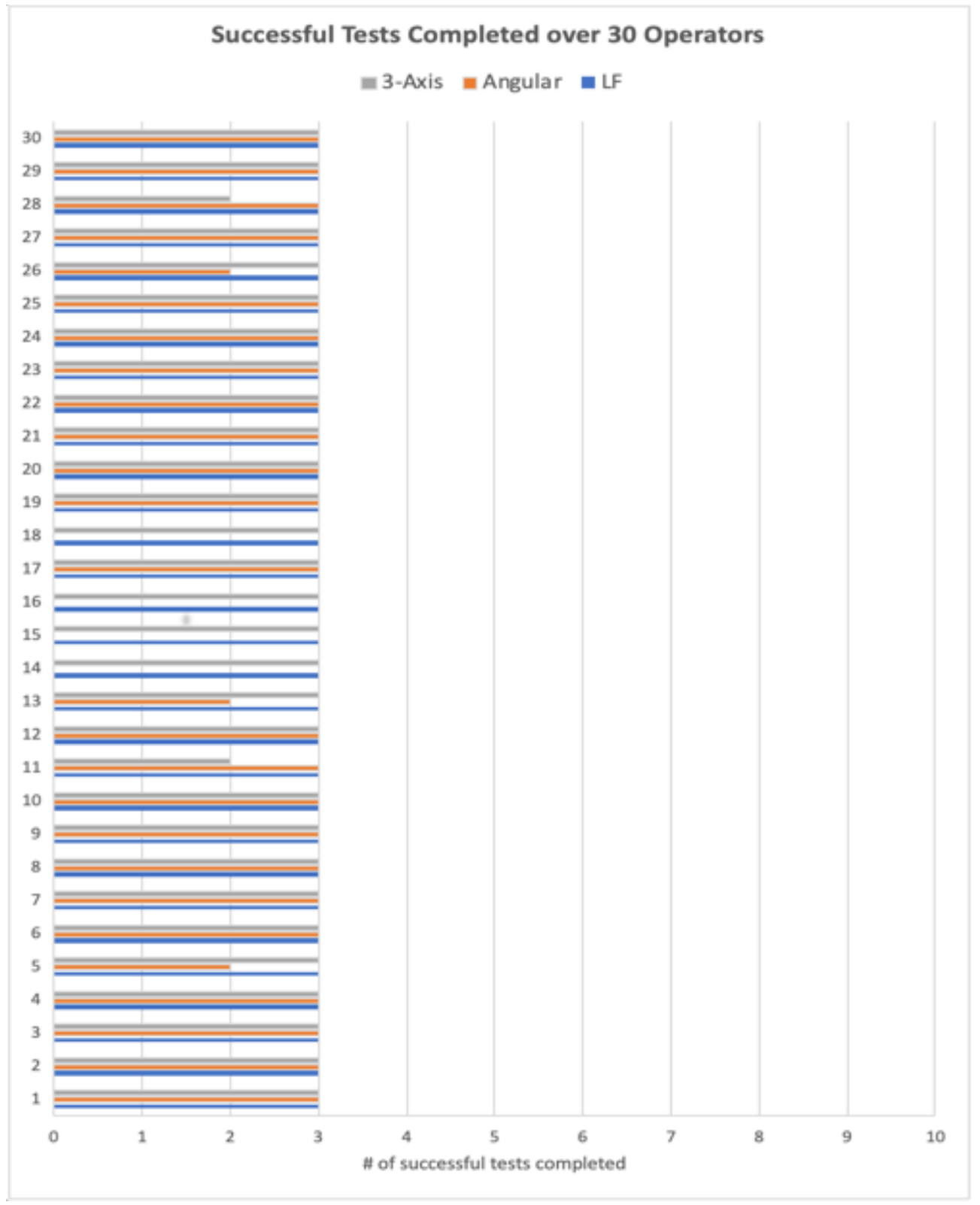

Figure 4.4.3 - Number of successful tests completed 
To better draw a meaningful conclusion about the overall operational efficiency of task performance, final results garnered from our experiments were also used to analyze the differences between the means and the standard deviations based on 30 tested operators which were calculated by the following equations:

$$
\bar{x}=\left(\sum_{i=1}^{N} x_{i}\right) / N
$$

Equation (3): Inputs Parameters: N, i, $x_{i}$

$$
s=\sqrt{\frac{1}{N-1} \sum_{i=1}^{N}\left(x_{i}-\bar{x}\right)^{2}}
$$

Equation (4): Inputs Parameters: N, i, $x_{i}, \bar{x}$

and can be summarized in Table 4.4:

Table 4.4 - Data comparison of the mean and the standard deviation based on 30 observations

\begin{tabular}{|c|c|c|}
\hline Test Method & Mean & STD \\
\hline $\begin{array}{c}\text { Joint by Joint } \\
\text { (Angular) }\end{array}$ & 122.14 & 61.65 \\
\hline $\begin{array}{c}\text { 3-Axis } \\
\text { (Cartesian) }\end{array}$ & 109.32 & 46.49 \\
\hline Leader-Follower & 77.82 & 27.81 \\
\hline
\end{tabular}

In the above table, the results were computed when using an input of 30 observations. Overall, both mean and standard deviation values become significantly less from Angular mode, 3-Axis mode, and the LF method, respectively. This indicates that the LF method produced markedly 
better performance for task completion speed and accuracy, especially when significant multitasking ${ }^{4}$ was required by the operator in order to achieve the remote manipulation goals.

Regardless of the negligible difference between the Angular and Cartesian modes, each method always takes more time for operators to switch between subsequent control modes while at the same time trying to align the end effector and the object to be grasped. During experiments, as illustrated in examples shown in Figure 4.2.1A and Figure 4.2.2A, operators had to switch more often between buttons or subsequent control modes in order to create different poses before bringing the end effector of the remote arm closer to target objects. The advanced solution is to directly interact with the operator arm by physically pushing or pulling on some link while at the same time each corresponding link of the remote arm can be synchronized, respectively. To overcome this challenge, the LF approach was introduced. This simple method requires no indepth operator training or expertise, and it also provides more natural remote control and a better level of convenience. Although the LF method might not be the best one yet, it can be used to improve remote control over other test methods discussed in this research.

Even though the test methods applied and results presented in this thesis do not apply all possible test scenarios made available as standard test methods by NIST, experimental results and graphical test forms associated with each test method provide a clear understanding of the robot's capabilities in order to facilitate side-by-side comparisons. The overall test results, from 30 operators, are used to support that the conclusions reached by this thesis are repeatable over various test methods.

\footnotetext{
${ }^{4}$ Several control strategies required the operator to perform certain non-obvious tasks to move individual components of the arm in order to move the entire arm. We argue that this is a form of significant multitasking.
} 


\subsection{Summary}

Overall, reported findings and data comparisons from different test methods presented in this chapter are helpful demonstrating that the most effective way of controlling a robot arm is the LF method in comparison to several common control strategies in an abstract test environment simulating common tasks associated with bomb disposal robot arms. When comparing the average time per task and the number of successful completions per test mode, we conclude that, by applying the LF method, remote robot manipulation system allows operators to perform more effectively once they have been trained on how to use it. In fact, remote manipulation tasks generally take operators more time to finish because operators have to think and decide which button or control must be activated next after each movement in order to achieve a desired movement in the robot arm. The LF method eliminates this additional step as the local arm is the only control required. 


\section{Chapter 5}

\section{Conclusion and Future Work}

\subsection{Conclusion}

The Leader-Follower method presented in this thesis allows operators a different method to more effectively manipulate a remote arm by imitating the exact motion on another arm. Using the standard metrics described in chapter 3 and the results attained from different test methods in chapter 4, we successfully demonstrated the efficacy of the LF control metaphor in relation to other control strategies when deploying robot arms for grasping and dropping objects in an indoor environment.

Overall, the Leader-Follower method has been recognized by most operators as one of the most advantageous methods because it lessens the cognitive load of operators, given the manipulation takes place remotely and operators are not able to directly view actual movements of the remote arm. During the experiments, a series of exercises based on various test methods were evaluated to capture the best possible performance for comparisons. While it is beyond the scope of this thesis to judge how fast training was assimilated by each operator, it was observed, however, that operators took less than the amount of time allocated to learn the LF method while a number of operators took longer to practice the other control strategies. The LF method is not just advantageous, but it is also flexible when performing numerous intricate movements. Although individual test for each method typically took less than 5 minutes, the overall average time per task and the average total time needed to complete the same tasks are much less when using the LF method. Regardless of different situations, the LF method helped operators successfully 
synchronize the motion of the remote arm with the operator arm without making as many attempts as with other test methods.

Even though operators, using the three test methods presented in this thesis, were always able to successfully accomplish the remote robot manipulation tasks, each of these methods has its own limitations. When using the 3-Axis mode, operators were able to move the robot arm around naturally but were not allowed to send any commands that go beyond the range limit of each actuator or each finger. On the other hand, operators can manually move each joint with a specific degree input when the Angular mode is enabled, but it does take some operators some time to figure out or arrange a set of joint positions required to form a desired pose (position and/or orientation) of a remote robot arm. Meanwhile, final results presented in this thesis evidently affirm that the deployment of robot arms for the object manipulation task can be achieved much quicker by manually pushing or pulling on some link around the arm when using the LF method. Nevertheless, as with other test methods, there are some limitations to the LF method. One of those limitations includes a short-time delay because the data input from each joint and each finger of the operator arm, in Cartesian coordinates, needs to be respectively translated into a new set of $\mathrm{x}$, $\mathrm{y}, \mathrm{z}$ coordinates required to move the remote arm.

In fact, human factors are primarily involved in the greater outcomes of different control strategies, including self-interpretation, imagination, and intuition. Even though the Leader-Follower method has been found to be an enhanced remote-control technique, operators might experience some difficulties due to the following challenges: accuracy, practicability, fixed quad displays and spurious states. In other words, the LF method does require operators to have the ability to expeditiously examine different situations of a remote arm through a quad display in order to achieve the best performance. Therefore, it is crucial to have some quick training for operators 
before entering into actual experiments so that they can gain some basic concepts of replicating movements between the operator arm and the remote arm.

Although the proposed standard metrics presented in this thesis do not address all challenges which can be found during Response Robot Evaluation Exercises developed by NIST, using the proposed tests allows us to remark different levels of difficulty. At the very least, final comparisons can be used to measure performance among different test methods discussed in this research. Despite operators having successfully trained themselves prior to each test mode, data comparisons of different test methods demonstrated that there is a higher rate of success for those who completed with the LF method. With a final glance at the average time of successful tasks, the LF method can be decidedly faster and more accurate than other test methods. As a final conclusion, we successfully demonstrated that, when using reported findings to compare the relative effectiveness of different ways of controlling a remote robot arm, the LF method provides operators with a more flexible remote-control technique while at the same time achieving the best performance. 


\subsection{Future Work}

The Kinova robot arm we used in this thesis allows us to remotely manipulate risky tasks through a desktop PC or a laptop using a wired connection. The operational environments may change, this upgrading the system to be controlled wirelessly via a network or by using a Bluetooth connection would provide more flexibility for future testing. Subsequently, a wireless system can support greater distances while at the same time providing a flexible and intuitive remote control.

The implementation of the Leader-Follower method is fulfilled via the Kinova API. Even though the LF method is handy for remote manipulation, we experience a very short period of delay each time operators send a command. As a result, this slightly affects the overall performance of humanrobot interaction controls. Sometimes, this also causes some confusion for operators if they try to move one joint that goes beyond an acceptable range limit, the joint automatically moves back to its previous position. Each robotic arm presented in this thesis has a maximum load which it can lift, push, pull, and manipulate. Generally, we look forward to seeing future work that will make use of our existing work and experience to improve the LF method so that human-robot interaction controls can be carried out better.

In addition to the technical limitations mentioned above, there are not many programming languages for developers to choose when the API option is selected. Currently, $\mathrm{C}++$ is the only supported programming language. Although it is possible to switch to Python or MATLAB, these are not officially supported. However, as the LF method has been shown to outperform various other common control strategies, it may prove to be a better strategy to simply use twinned robot arms from a different manufacturer. 


\section{Appendix A - Feedback on Control Mode}

If there are no blue lights shown, it means the controller is currently disabled. Either one of the following actions should be taken:

- Toggle the On/Off button on the joystick

- Bring the $\mathrm{MICO}^{2}$ arm back to its HOME position

The following figures show a detail of all feedback that might appear on control mode:

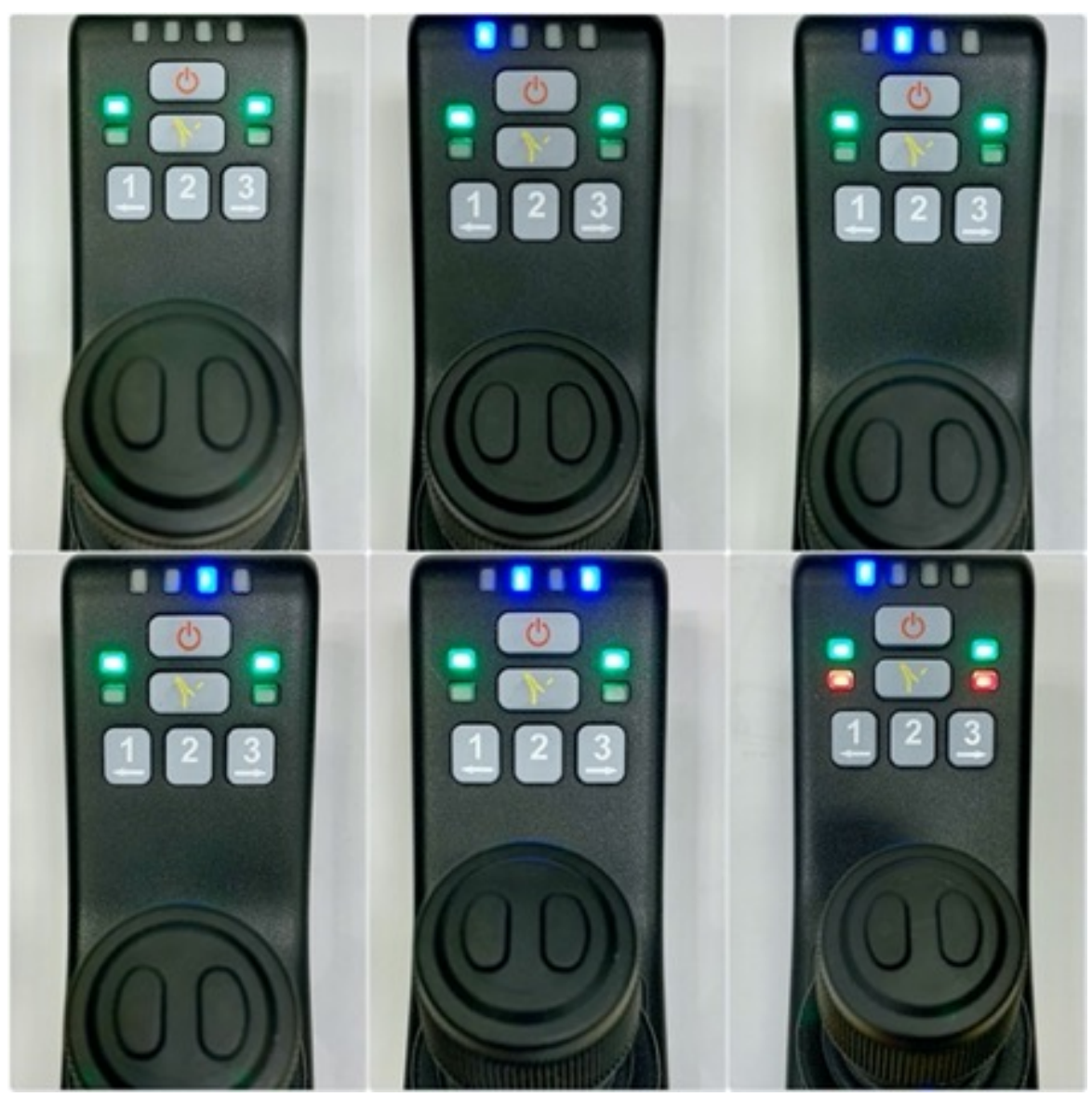

Figure A - Kinova joystick with different statuses of blue LEDs feedback 


\section{Appendix B - Fixed Camera Views of the Apparatus}

An actual view from all three cameras through an LCD display which assists operators in knowing the current location of the remote $\mathrm{MICO}^{2}$ arm on scene (Figure B). This is very helpful for operators because they are not able to see actual movements of the remote arm but through a quad display.

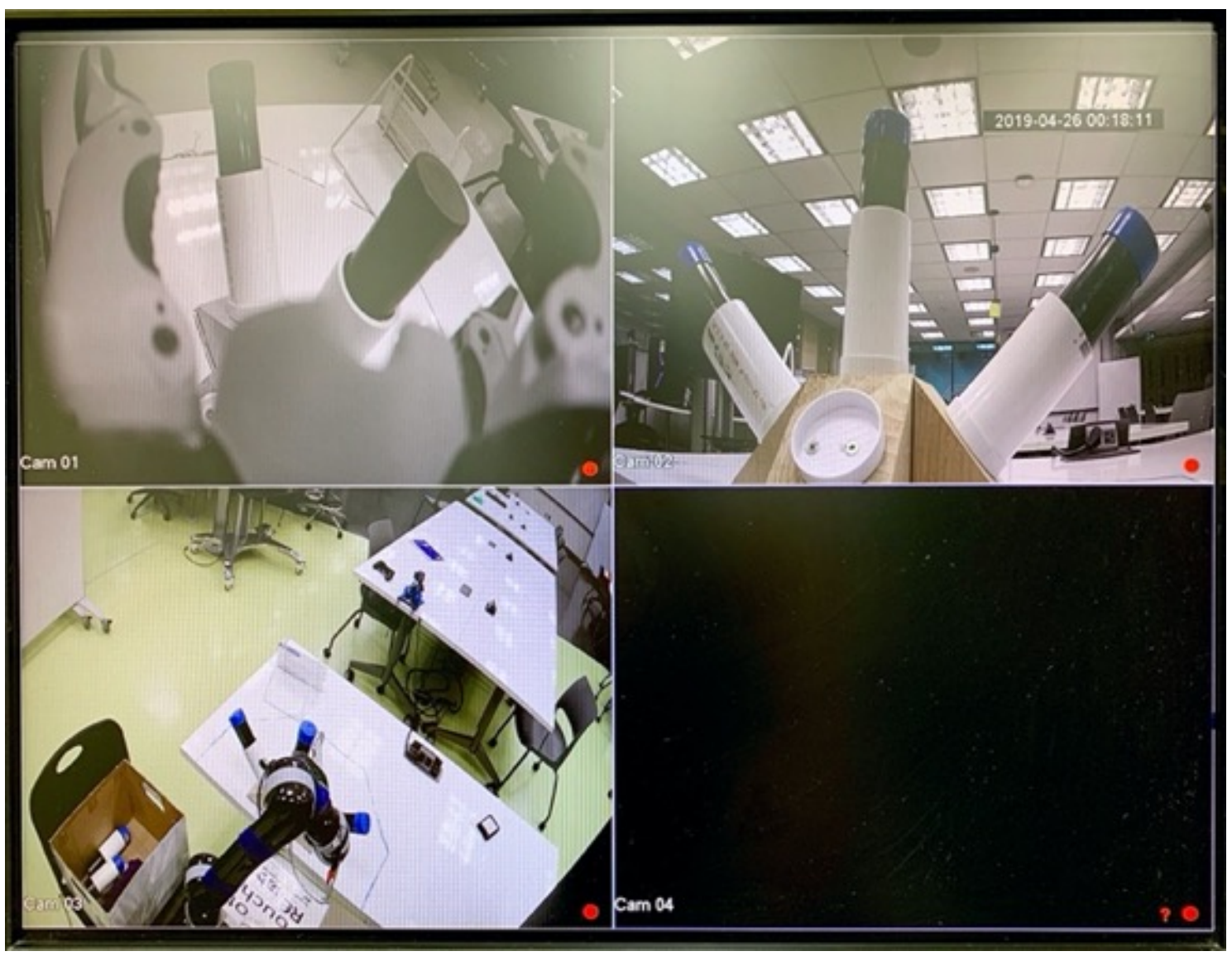

Figure B - A quad display providing views of the apparatus from 3 cameras 


\section{Appendix C - Software Limitations of Actuators and Fingers}

By default, self-collisions and protection zones are automatically enabled while controlling the robot arm in Cartesian mode, but not in Angular mode. Regardless of which operation mode is being used, to ensure the safety of the robot arm's operations, joint and finger limits are also considered. The following tables [144] indicate the minimum and maximum positions of each specific actuator and finger of a 6 DOF curved wrist arm that operators should operate within its range limit; otherwise it will stop moving.

Table C1 - Kinova $\mathrm{MICO}^{2}$ arm software limitations of actuators

\begin{tabular}{|c|c|c|}
\hline Actuator & $\operatorname{Min}\left({ }^{\circ}\right)$ & $\operatorname{Max}\left({ }^{\circ}\right)$ \\
\hline 1 & -10000 & 10000 \\
\hline 2 & 50 & 310 \\
\hline 3 & 19 & 341 \\
\hline 4 & -10000 & 10000 \\
\hline 5 & -10000 & 10000 \\
\hline 6 & -10000 & 10000 \\
\hline
\end{tabular}

Table C2 - Kinova $\mathrm{MICO}^{2}$ arm software limitations of fingers

\begin{tabular}{|c|c|c|}
\hline Finger \# & $\operatorname{Min}\left({ }^{\circ}\right)$ & $\operatorname{Max}\left({ }^{\circ}\right)$ \\
\hline 1 & 0 & $6800(18.9 \mathrm{~mm})$ \\
\hline 2 & 0 & $6800(18.9 \mathrm{~mm})$ \\
\hline 3 & 0 & $6800(18.9 \mathrm{~mm})$ \\
\hline
\end{tabular}




\section{Appendix D - Singularity Auto-avoidance Behaviour}

As discussed in section 4.2.2, Kinova $\mathrm{MICO}^{2}$ arm presented in this research might enter into a state that one or more degrees of freedom will be lost. This typically happens when operators, by any chance, send a command which may cause a collision between either the end effector and the base or the end effector and the arm itself. This behaviour is known as singularity avoidance and can be disabled by changing the parameter settings of the ActiveAutomaticCollisionAvoidance API function.

A typical robot arm in boundary singularity situation, as depicted in Figure D1 below, is at full reach. Therefore, it is impossible to bring elbow joint at $180^{\circ}$ as operators are not able to move any further in the direction it is currently reaching out.

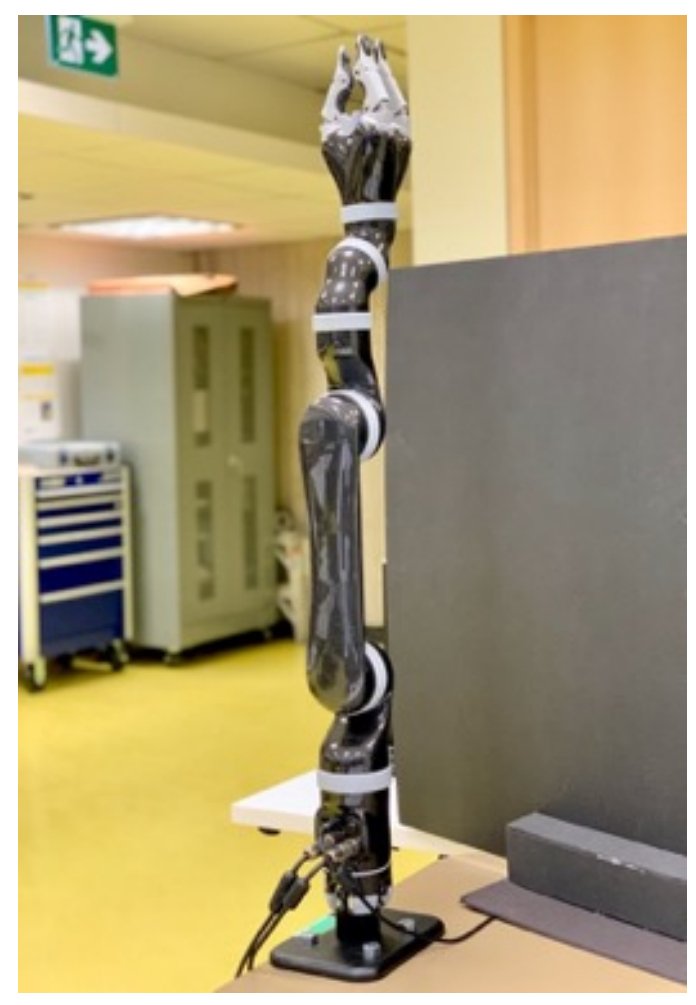

Figure D1 - Kinova 6 DOF $\mathrm{MICO}^{2}$ arm with boundary singularity 
As an example of the following (wrist-over-base singularity), it is impossible to bring the wrist inside a virtual cylinder located around its base when the wrist joint is already aligned with its first joint axis.

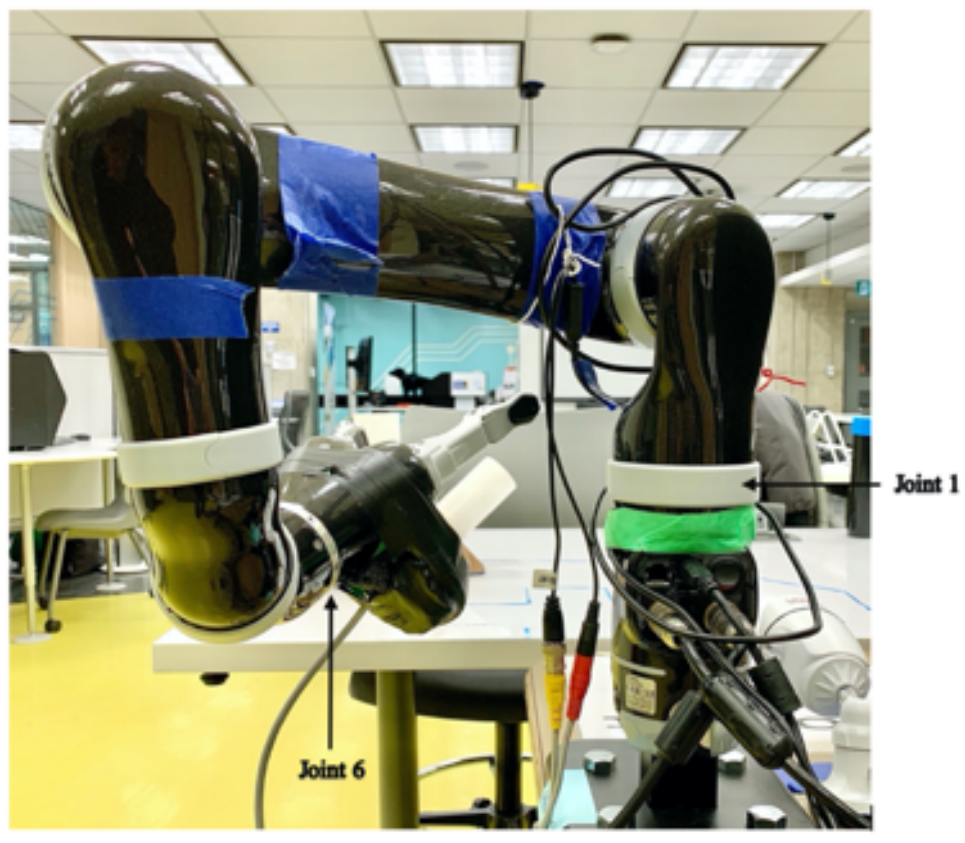

Figure D2 - Kinova 6 DOF MICO $^{2}$ arm with wrist-over-base singularity 


\section{Bibliography}

[1] K. Keerthana Rathan, A. Ajith, S. Aswathi and V. M. Silpa, "Survey of robotic arm controlling techniques," 2017 International Conference on Intelligent Sustainable Systems (ICISS), Palladam, 2017, pp. 759-763.

[2] A. Davids, "Urban search and rescue robots: from tragedy to technology," in IEEE Intelligent Systems, vol. 17, no. 2, pp. 81-83, March-April 2002.

[3] D. K. Richardson, "Robots to the rescue?," in Engineering \& Technology, vol. 6, no. 4, pp. 5254, May 2011.

[4] R. R. Murphy, "Trial by fire [rescue robots]," in IEEE Robotics \& Automation Magazine, vol. 11, no. 3, pp. 50-61, Sept. 2004.

[5] Wikipedia, "Robotic arm — Wikipedia, The Free Encyclopedia." 2020.

[6] K. Shaw and J. Santagate, "Top Robotics Companies Continue to Innovate, Grow Market," 2019 Robotics Business Review.

[7] "Products". Gerenga Co. , Ltd. http://www.gerenga.com/index.php?sub=products

[8] "Robotic arms". Kinova Inc., https://www.kinovarobotics.com/en/products/robotic-arm-series [9] “Moxi”. Diligent Robotics Inc., https://diligentrobots.com/moxi

[10] W. Schoor, M. Förster and A. Radetzky, "Realistic training simulations of explosive ordnance disposal \& improvised explosive device disposal robots," IEEE $10^{\text {th }}$ International Conference on Industrial Informatics, Beijing, 2012, pp. 875-880.

[11] A. K. B. Motaleb, M. B. Hoque and M. A. Hoque, "Bomb disposal robot," 2016 International Conference on Innovations in Science, Engineering and Technology (ICISET), Dhaka, 2016, pp. 1-5.

[12] S. Costo and R. Molfino, "A new robotic unit for onboard airplanes bomb dispoal," $35^{\text {th }}$ International Symposium on Robotics ISR 2004, Paris, March 2004, pp. 23-26.

[13] B. Wei, J. Gao, J. Zhu and K. Li, "Design of a Large Explosive Ordnance Disposal Robot," 2009 Second International Conference on Intelligent Computation Technology and Automation, Changsha, Hunan, 2009, pp. 403-406.

[14] D. Herald, "DRDO's robots to handle explosives," [Online]. Available: https://www.icast.org.in/news/2009/feb09/feb18da.pdf, 2009.

[15] J. Smith, "Police robots could save a lot of lives if we weren't afraid to use them," [Online]. Available: https://www.businessinsider.com/police-robots-could-save-lives-2016-7, 2016. 
[16] A. Beale, "A New Weapon for SWAT Teams: Bomb-Squad Robots," [Online]. Available: https://www.vice.com/en_us/article/ppxdn9/a-new-weapon-for-swat-teams-bomb-squad-robots$522,2015$.

[17] G. Zorpette, "Lessons From the New York Bombing," [Online]. Available: https://spectrum.ieee.org/tech-talk/at-work/test-and-measurement/lessons-from-the-new-yorkbombing, 2016.

[18] S. Cherry, "IEDs in Iraq, Afghanistan, and Boston," [Online]. Available: https://spectrum.ieee.org/podcast/at-work/test-and-measurement/ieds-in-iraq-afghanistan-andboston, 2013.

[19] D. M. Blyth, H. C. Yun, D. R. Tribble and C. K. Murray, "Lessons of War: Combat-related Injury Infections during the Vietnam War and Operation Iraqi and Enduring Freedom," The Journal of Trauma and Acute Care Surgery, 2015, vol 79, 4 Suppl 2, S227-35.

[20] F. G. Rando, "The Road Ahead: The Constant Threat of the Roadside Bomb," [Online]. Available: http://www.cbrneportal.com/the-road-ahead-the-constant-threat-of-the-roadside-bomb, 2015.

[21] Ting Li and Qiongwei Ye, "New thoughts about supply chain security after 9-11 terrorist attack," 2008 IEEE International Conference on Service Operations and Logistics, and Informatics, Beijing, 2008, pp. 2397-2400.

[22] R. R. Murphy, "Human-robot interaction in rescue robotics," in IEEE Transactions on Systems, Man, and Cybernetics, Part C (Applications and Reviews), vol. 34, no. 2, pp. 138-153, May 2004.

[23] J. Casper and R. R. Murphy, "Human-robot interactions during the robot-assisted urban search and rescue response at the World Trade Center," in IEEE Transactions on Systems, Man, and Cybernetics, Part B (Cybernetics), vol. 33, no. 3, pp. 367-385, June 2003.

[24] J. L. Burke and R. R. Murphy, "Human-robot interaction in USAR technical search: two heads are better than one," RO-MAN 2004. 13 $3^{\text {th }}$ IEEE International Workshop on Robot and Human Interactive Communication (IEEE Catalog No.04TH8759), Kurashiki, Okayama, Japan, 2004, pp. 307-312.

[25] Z. Zhang, H. Guo, G. Nejat and P. Huang, "Finding Disaster Victims: A Sensory System for Robot-Assisted 3D Mapping of Urban Search and Rescue Environments," Proceedings 2007 IEEE International Conference on Robotics and Automation, Roma, 2007, pp. 3889-3894. 
[26] C. Beltran-Gonzalez et al., "Methods and techniques for intelligent navigation and manipulation for bomb disposal and rescue operations," 2007 IEEE International Workshop on Safety, Security and Rescue Robotics, Rome, 2007, pp. 1-6.

[27] A. Watanabe, H. Miura, M. Okugawa and K. Hatanaka, "Verification of Scenario for RobotAssisted Fire-Fighting and Rescue Operations," 2019 IEEE International Symposium on Safety, Security, and Rescue Robotics (SSRR), Würzburg, Germany, 2019, pp. 106-107.

[28] R. R. Murphy, "Marsupial and shape-shifting robots for urban search and rescue," in IEEE Intelligent Systems and their Applications, vol. 15, no. 2, pp. 14-19, March-April 2000.

[29] G. Zorpette, “Bomb squad diary,” in IEEE Spectrum, vol. 45, no. 10, pp. 40-47, Oct. 2008.

[30] D. Strellis and T. Gozani, "A Neutron Interrogation Based Vehicle Borne IED Detection System for Checkpoint Security," 2008 IEEE Conference on Technologies for Homeland Security, Waltham, MA, 2008, pp. 395-399.

[31] A. Jousselme and P. Maupin, "Uncertainty representations for a Vehicle-Borne IED surveillance problem," 2012 15th International Conference on Information Fusion, Singapore, 2012, pp. 1482-1489.

[32] J. Dezert and F. Smarandache, "Threat assessment of a possible vehicle-born improvised explosive device using dsmt," 2010. [Online]. Available: http://vixra.org/pdf/1008.0026v2.pdf. [Accessed Dec. 17, 2019].

[33] R. Deming, R. Ilin and S. MacIntosh, "Deep learning for fusing multi-sensor person-borne IED data," 2017 IEEE International Symposium on Technologies for Homeland Security (HST), Waltham, MA, 2017, pp. 1-5.

[34] R. F. Fahy, "The Study of Occupant Behavior During the World Trade Center Evacuation Preliminary Report of Results," [Online]. Available:

https://tsapps.nist.gov/publication/get_pdf.cfm?pub_id=909237. [Accessed Dec. 20, 2019].

[35] J. V. Parachini, “The World Trade Center Bombers (1993),” [Online]. Available:

https://www.nonproliferation.org/wp-content/uploads/2016/05/world_trade_center_bombers.pdf. [Accessed Dec. 21, 2019].

[36] K. Osuka, R. Murphy and A. C. Schultz, "USAR competitions for physically situated robots," in IEEE Robotics \& Automation Magazine, vol. 9, no. 3, pp. 26-33, Sept. 2002.

[37] P. Gilbert-Bonner, "Terrorism, McVeigh, and the Oklahoma City Bombing," 2016.

[38] D. E. Hogan, J. F. Waeckerle, D. J. Dire and S. R. Lillibridge, "Emergency Department Impact of the Oklahoma City Terrorist Bombing," [Online]. Available: 
https://www.annemergmed.com/article/S0196-0644(99)70224-6/pdf. [Accessed Dec. 28, 2019]. [39] J. Hollingum, "Robots for the dangerous tasks," Industrial Robot, vol. 26, no. 3, January 1999, pp. 178-183.

[40] A Division of Monster Worldwide, "The Very First Bomb Disposal Robot," military.com, Jan. 15, 2014. [Online]. Available:

https://www.military.com/video/ammunition-and-explosives/explosive-ordnance-disposal/thefirst-bomb-disposal-robot/3059244734001 [Accessed Dec. 15, 2019].

[41] Sungchul Kang et al., "ROBHAZ-DT2: design and integration of passive double tracked mobile manipulator system for explosive ordnance disposal," Proceedings 2003 IEEE/RSJ International Conference on Intelligent Robots and Systems (IROS 2003) (Cat. No.03CH37453), Las Vegas, NV, USA, 2003, pp. 2624-2629 vol.3.

[42] B. Wei, J. Gao, J. Zhu and K. Li, "Design of a Large Explosive Ordnance Disposal Robot," 2009 Second International Conference on Intelligent Computation Technology and Automation, Changsha, Hunan, 2009, pp. 403-406.

[43] I. Roderick, "Considering the fetish value of EOD robots: How robots save lives and sell war," International Journal of Cultural Studies, vol. 13, no. 3, May, 2010, pp. 235-253.

[44] C. Saliba, M. K. Bugeja, S. G. Fabri, M. D. Castro, A. Mosca and M. Ferre, "A Training Simulator for Teleoperated Robots Deployed at CERN," $201815^{\text {th }}$ International Conference on Informatics in Control, Automation and Robotics, 2018.

[45] R. Bogue, "Search and Rescue and Disaster Relief Robots: Has Their Time Finally Come?," Industrial Robot, vol. 43, no. 2, pp. 138-143, 2016.

[46] W. Schoor, M. Förster and A. Radetzky, "Realistic training simulations of explosive ordnance disposal \& improvised explosive device disposal robots," IEEE 10th International Conference on Industrial Informatics, Beijing, 2012, pp. 875-880.

[47] M. Fracchia et al., "Low-cost explosive ordnance disposal robot for deployment in Southeast Asia," 2015 IEEE Canada International Humanitarian Technology Conference (IHTC2015), Ottawa, ON, 2015, pp. 1-4.

[48] M. Fachot, "From bomb disposal to waste disposal...," June 2011. [Online]. Available: https://iecetech.org/issue/2011-06/From-bomb-disposal-to-waste-disposal. [Accessed Dec. 28, 2019].

[49] S. Arimoto, Ji-Hun Bae, H. Hashiguchi and R. Ozawa, "Why does surplus DOF of robot fingers enhance dexterity of object manipulation: analysis and simulation of overall fingers-object 
dynamics," IEEE International Conference on Robotics and Automation, 2004. Proceedings. ICRA '04. 2004, New Orleans, LA, USA, 2004, pp. 2936-2943 Vol.3.

[50] H. W. Stone and G. Edmonds, "HAZBOT: a hazardous materials emergency response mobile robot," Proceedings 1992 IEEE International Conference on Robotics and Automation, Nice, France, 1992, pp. 67-73 vol.1.

[51] D. Voth, "A new generation of military robots," in IEEE Intelligent Systems, vol. 19, no. 4, pp. 2-3, July-Aug. 2004.

[52] N. Sharkey, "Cassandra or False Prophet of Doom: AI Robots and War," in IEEE Intelligent Systems, vol. 23, no. 4, pp. 14-17, July-Aug. 2008.

[53] United States. Federal Emergency Management Agency, "Small Bomb Disposal Robot -Vanguard MKII-T: Project Summary," hsdl.org, March 2005. [Online]. Available:

https://www.hsdl.org/?abstract\&did=484061. [Accessed Jan. 4, 2020]

[54] R. Murphy, J. Blitch and J. Casper, "AAAI/robocup-2001 urban search and rescue events: Reality and competition,” AI Magazine, vol. 23, (1), pp. 37, Spring 2002.

[55] E. Guizzo, "Rescue-robot show-down," in IEEE Spectrum, vol. 51, no. 1, pp. 52-55, January 2014.

[56] J. A. Bagnell, D. Bradley, D. Silver, B. Sofman and A. Stentz, "Learning for Autonomous Navigation," in IEEE Robotics \& Automation Magazine, vol. 17, no. 2, pp. 74-84, June 2010.

[57] C. E. Agüero et al., "Inside the Virtual Robotics Challenge: Simulating Real-Time Robotic Disaster Response," in IEEE Transactions on Automation Science and Engineering, vol. 12, no. 2, pp. 494-506, April 2015.

[58] K. Knoedler, V. Dimitrov, D. Conn, M. A. Gennert and T. Padır, "Towards supervisory control of humanoid robots for driving vehicles during disaster response missions," 2015 IEEE International Conference on Technologies for Practical Robot Applications (TePRA), Woburn, MA, 2015, pp. 1-6.

[59] T. Bagosi, K. V. Hindriks and M. A. Neerincx, "Ontological reasoning for human-robot teaming in search and rescue missions," $201611^{\text {th }}$ ACM/IEEE International Conference on Human-Robot Interaction (HRI), Christchurch, 2016, pp. 595-596.

[60] I. R. Nourbakhsh, K. Sycara, M. Koes, M. Yong, M. Lewis and S. Burion, "Human-robot teaming for search and rescue," in IEEE Pervasive Computing, vol. 4, no. 1, pp. 72-79, Jan.-March 2005. 
[61] C. Willms, C. Houy, J. Rehse, P. Fettke and I. Kruijff-Korbayová, "Team communication processing and process analytics for supporting robot-assisted emergency response," 2019 IEEE International Symposium on Safety, Security, and Rescue Robotics (SSRR), Würzburg, Germany, 2019, pp. 216-221.

[62] S. Reed, J. Wood and C. Haworth, "The detection and disposal of IED devices within harbor regions using AUVs, smart ROVs and data processing/fusion technology," 2010 International WaterSide Security Conference, Carrara, 2010, pp. 1-7.

[63] M. Nijim, "Multitasking intelligent surveillance and first response system," 2016 IEEE Symposium on Technologies for Homeland Security (HST), Waltham, MA, 2016, pp. 1-6.

[64] J. M. Sullivan, "Revolution or evolution? The rise of the UAVs," Proceedings. 2005 International Symposium on Technology and Society, 2005. Weapons and Wires: Prevention and Safety in a Time of Fear. ISTAS 2005., Los Angeles, CA, USA, 2005, pp. 94-101.

[65] J. Connell, G. M. Schechtman and B. Hasty, "KM Issues with Retiring and Transient Explosive Ordnance Disposal (EOD) Airmen and Their Impact on Air Force EOD Response Capabilities," 2012 45th Hawaii International Conference on System Sciences, Maui, HI, 2012, pp. 3709-3718.

[66] J. M. Peschel and R. R. Murphy, "On the Human-Machine Interaction of Unmanned Aerial System Mission Specialists," in IEEE Transactions on Human-Machine Systems, vol. 43, no. 1, pp. 53-62, Jan. 2013.

[67] H. C. Nguyen, R. Amorim, J. Wigard, I. Z. KováCs, T. B. Sørensen and P. E. Mogensen, "How to Ensure Reliable Connectivity for Aerial Vehicles Over Cellular Networks," in IEEE Access, vol. 6, pp. 12304-12317, 2018.

[68] A. Garcia and K. Ghose, "Autonomous indoor navigation of a stock quadcopter with offboard control," 2017 Workshop on Research, Education and Development of Unmanned Aerial Systems (RED-UAS), Linkoping, 2017, pp. 132-137.

[69] A. Deleforge, D. Di Carlo, M. Strauss, R. Serizel and L. Marcenaro, "Audio-Based Search and Rescue With a Drone: Highlights From the IEEE Signal Processing Cup 2019 Student Competition [SP Competitions]," in IEEE Signal Processing Magazine, vol. 36, no. 5, pp. 138144, Sept. 2019.

[70] F. L. Afriansyah, N. Muna, I. Widiastuti, N. Z. Fanani and F. E. Purnomo, "Image Mapping Detection of Green Areas Using Speed Up Robust Features," 2019 International Conference on 
Computer Science, Information Technology, and Electrical Engineering (ICOMITEE), Jember, Indonesia, 2019, pp. 165-168.

[71] "NIST Performance Tests for Aerial Response Robots Become National Standard," Dec. 4, 2018. [Online]. Available:

https://www.nist.gov/news-events/news/2018/12/nist-performance-tests-aerial-response-robotsbecome-national-standard. [Accessed Jan. 8, 2020]

[72] L. Hull, R. Riley, L. Wright and C. Yoder, "The implementation of Unmanned Vehicles in Search and Rescue Operations," Oct. 21, 2019. [Online]. Available:

https://storymaps.arcgis.com/stories/0e44e3407a084c11a778c2491cf9f273. [Accessed Jan. 6, 2020]

[73] T. M. Cabreira, K. Kappel, L. B. de Brisolara and P. R. Ferreira, "An Energy-Aware RealTime Search Approach for Cooperative Patrolling Missions with Multi-UAVs," 2018 Latin American Robotic Symposium, 2018 Brazilian Symposium on Robotics (SBR) and 2018 Workshop on Robotics in Education (WRE), Joao Pessoa, 2018, pp. 254-259.

[74] A. Viseras, J. Marchal, M. Schaab, J. Pages and L. Estivill, "Wildfire Monitoring and Hotspots Detection with Aerial Robots: Measurement Campaign and First Results," 2019 IEEE International Symposium on Safety, Security, and Rescue Robotics (SSRR), Würzburg, Germany, 2019, pp. 102-103.

[75] T. Shaneyfelt, M. A. Joordens, K. Nagothu and M. Jamshidi, "RF communication between surface and underwater robotic swarms," 2008 World Automation Congress, Hawaii, HI, 2008, pp. $1-6$.

[76] M. Hussain, J. Ahmad, I. Tufail, M. Hussain and F. Rehman, "Design and Development of Underwater Remotely Operated Locomotion Robotic System," 2018 International Conference on Computational Techniques, Electronics and Mechanical Systems (CTEMS), Belgaum, India, 2018, pp. 235-239.

[77] S. A. Fattah, F. Abedin, M. N. Ansary, M. A. Rokib, N. Saha and C. Shahnaz, "R3Diver: Remote robotic rescue diver for rapid underwater search and rescue operation," 2016 IEEE Region 10 Conference (TENCON), Singapore, 2016, pp. 3280-3283.

[78] S. Reed, J. Wood and C. Haworth, "The detection and disposal of IED devices within harbor regions using AUVs, smart ROVs and data processing/fusion technology," 2010 International WaterSide Security Conference, Carrara, 2010, pp. 1-7.

[79] "What Are Underwater ROVs \& What Are They Used For?,” Deep Trekker Inc., 
https://www.deeptrekker.com/resources/underwater-rovs. [Accessed Jan. 8, 2020]

[80] W. A. Pineda MuñOz, A. Gauthier Sellier and S. GomàRiz Castro, "The Predictive Functional Control and the Management of Constraints in GUANAY II Autonomous Underwater Vehicle Actuators," in IEEE Access, vol. 6, pp. 22353-22367, 2018.

[81] E. Cardenaz and J. G. Ramirez-Torres, “Autonomous navigation of unmanned aerial vehicles guided by visual features of the terrain," 2015 12th International Conference on Electrical Engineering, Computing Science and Automatic Control (CCE), Mexico City, 2015, pp. 1-6.

[82] B. Luk, K. Liu, A. Collie, D. Cooke and S. Chen, "Tele-operated climbing and mobile service robots for remote inspection and maintenance in nuclear industry," Industrial Robot: An International Journal, vol. 33, no. 3, pp. 194-204, 2006.

[83] S. Tadokoro et al., "The RoboCup-Rescue project: a robotic approach to the disaster mitigation problem," Proceedings 2000 ICRA. Millennium Conference. IEEE International Conference on Robotics and Automation. Symposia Proceedings (Cat. No.00CH37065), San Francisco, CA, USA, 2000, pp. 4089-4094 vol.4.

[84] Ø. Netland and A. Skavhaug, "Prototyping and evaluation of a telerobot for remote inspection of offshore wind farms," 2012 2nd International Conference on Applied Robotics for the Power Industry (CARPI), Zurich, 2012, pp. 187-192.

[85] M. Di Castro, M. L. B. Tambutti, M. Ferre, R. Losito, G. Lunghi and A. Masi, "i-TIM: A Robotic System for Safety, Measurements, Inspection and Maintenance in Harsh Environments," 2018 IEEE International Symposium on Safety, Security, and Rescue Robotics (SSRR), Philadelphia, PA, 2018, pp. 1-6.

[86] J. S. Artal-Sevil and J. L. Montañés, "Development of a robotic arm and implementation of a control strategy for gesture recognition through Leap Motion device," 2016 Technologies Applied to Electronics Teaching (TAEE), Seville, 2016, pp. 1-9.

[87] F. Weichert et al., "Analysis of the Accuracy and Robustness of the Leap Motion Controller," Sensors, Basel, Switzerland, vol. 13, no. 5, pp. 6380-6393, May 2013.

[88] L. Vani and R. A. Reddy, "Robotic Arm Manipulation Using Leap Motion Controller," International Journal of Advanced Technology in Engineering and Science, Narsapur, Medak, vol. 3, no. 1, August 2015.

[89] Y. Lin, S. Song and M. Q. -. Meng, "The implementation of augmented reality in a robotic teleoperation system," 2016 IEEE International Conference on Real-time Computing and Robotics (RCAR), Angkor Wat, 2016, pp. 134-139. 
[90] T. D. White, G. Fraser and G. J. Brown, "Modelling Hand Gestures to Test Leap Motion Controlled Applications," 2018 IEEE International Conference on Software Testing, Verification and Validation Workshops (ICSTW), Vasteras, 2018, pp. 204-213.

[91] V. Kiselev, M. Khlamov and K. Chuvilin, "Hand Gesture Recognition with Multiple Leap Motion Devices," 2019 24th Conference of Open Innovations Association (FRUCT), Moscow, Russia, 2019, pp. 163-169.

[92] P. R. Hayden, "Unmanned Systems: A Lab-Based Robotic Arm for Grasping Phase II," Master's thesis, Naval Postgraduate School, December 2016. [Online]. Available: http://hdl.handle.net/10945/51716. [Accessed Jul. 15, 2019]

[93] D. Bassily, C. Georgoulas, J. Guettler, T. Linner and T. Bock, "Intuitive and Adaptive Robotic Arm Manipulation using the Leap Motion Controller," ISR/Robotik 2014; 41st International Symposium on Robotics, Munich, Germany, 2014, pp. 1-7.

[94] A. Jacinto, "Unmanned Systems: A Lab-Based Robotic Arm for Grasping," Master's thesis, Naval Postgraduate School, June 2015. [Online]. Available: http://hdl.handle.net/10945/45879. [Accessed Jul. 13, 2019]

[95] R. Ozawa and N. Ueda, "Supervisory control of a multi-fingered robotic hand system with data glove," 2007 IEEE/RSJ International Conference on Intelligent Robots and Systems, San Diego, CA, 2007, pp. 1606-1611.

[96] R. Sadeghian, P. Sedigh, P. Azizinezhad, S. Shahin and M. T. Masouleh, "Design, Development and Control of a Three Flexible-Fingers Gripper Based On Hand Gesture," 2018 6th RSI International Conference on Robotics and Mechatronics (IcRoM), Tehran, Iran, 2018, pp. 359-363.

[97] Y. Yoshimura and R. Ozawa, "A supervisory control system for a multi-fingered robotic hand using datagloves and a haptic device," 2012 IEEE/RSJ International Conference on Intelligent Robots and Systems, Vilamoura, 2012, pp. 5414-5419.

[98] R. Oliveira, R. Resende, F. Soares, A. Calado and P. Leite, "Mimicking Human Movement with Robots: Control of an Anthropomorphic Robotic Arm using a Glove-Based System as an Educational Tool," 2019 6th International Conference on Control, Decision and Information Technologies (CoDIT), Paris, France, 2019, pp. 79-84.

[99] M. Fischer, P. van der Smagt and G. Hirzinger, "Learning techniques in a dataglove based telemanipulation system for the DLR hand," Proceedings. 1998 IEEE International Conference on Robotics and Automation (Cat. No.98CH36146), Leuven, Belgium, 1998, pp. 1603-1608 vol.2. 
[100] R. H. Palacios, "Robotic Arm Manipulation Laboratory with a Six Degree of Freedom JACO Arm," Master's thesis, Naval Postgraduate School, December 2015. [Online]. Available: http://hdl.handle.net/10945/45879. [Accessed Jul. 17, 2019]

[101] W. Uttal, “Teleoperators,” Sci. Amer., vol. 261, pp. 74-79, Dec. 1989.

[102] Y. K. Chao, Y. F. Zhou and Y. L. Xu, "Mobile robotic control system based on master-slave teleoperate mechanical arm," 2013 25th Chinese Control and Decision Conference (CCDC), Guiyang, 2013, pp. 3195-3198.

[103] S. Kumra, S. Mehta and R. Singh, "Development of anthropomorphic multi-D.O.F masterslave manipulator," 2013 3rd IEEE International Advance Computing Conference (IACC), Ghaziabad, 2013, pp. 1631-1635.

[104] J. Kim, Y. Choi and J. Lee, "Development of a master station for the remote control of rescue robots," 2012 12th International Conference on Control, Automation and Systems, JeJu Island, 2012, pp. 1994-1997.

[105] P. Payeur, C. Pasca, A. -. Cretu and E. M. Petriu, "Intelligent haptic sensor system for robotic manipulation," in IEEE Transactions on Instrumentation and Measurement, vol. 54, no. 4, pp. 1583-1592, Aug. 2005.

[106] Dongseok Ryu, Chang-Soon Hwang, Sungchul Kang, Munsang Kim and Jae-Bok Song, "Wearable haptic-based multi-modal teleoperation of field mobile manipulator for explosive ordnance disposal," IEEE International Safety, Security and Rescue Rototics, Workshop, 2005., Kobe, 2005, pp. 75-80.

[107] A. J. Johansson and J. Linde, "Using simple force feedback mechanisms as haptic visualization tools," IMTC/99. Proceedings of the 16th IEEE Instrumentation and Measurement Technology Conference (Cat. No.99CH36309), Venice, 1999, pp. 820-824 vol.2.

[108] W. Semere, M. Kitagawa and A. M. Okamura, "Teleoperation with sensor/actuator asymmetry: task performance with partial force feedback," 12th International Symposium on Haptic Interfaces for Virtual Environment and Teleoperator Systems, 2004. HAPTICS '04. Proceedings., Chicago, IL, USA, 2004, pp. 121-127.

[109] I. Yamano, K. Takemura, K. Endo and T. Maeno, "Method for controlling master-slave robots using switching and elastic elements," Proceedings 2002 IEEE International Conference on Robotics and Automation (Cat. No.02CH37292), Washington, DC, USA, 2002, pp. 1717-1722 vol.2. 
[110] S. Lee and H. S. Lee, "Teleoperator control system design with human in control loop and telemonitoring force feedback," [1992] Proceedings of the 31st IEEE Conference on Decision and Control, Tucson, AZ, USA, 1992, pp. 2674-2679 vol.3.

[111] N. Cauche, A. Delchambre, P. Rouiller, P. Helmer, C. Baur and R. Clavel, "Rotational forcefeedback wrist," Proceedings of the IEEE International Symposium on Assembly and Task Planning, 2003., Besancon, France, 2003, pp. 210-215.

[112] G. S. Gupta, S. C. Mukhopadhyay, C. H. Messom and S. N. Demidenko, "Master-Slave Control of a Teleoperated Anthropomorphic Robotic Arm with Gripping Force Sensing," in IEEE Transactions on Instrumentation and Measurement, vol. 55, no. 6, pp. 2136-2145, Dec. 2006.

[113] Wikipedia, "Kinematics — Wikipedia, The Free Encyclopedia." 2019.

[114] Yujie Cui, Pu Shi and Jianning Hua, "Kinematics analysis and simulation of a 6-DOF humanoid robot manipulator," 2010 2nd International Asia Conference on Informatics in Control, Automation and Robotics (CAR 2010), Wuhan, 2010, pp. 246-249.

[115] S. Wen, Z. Ma, S. Wen, Y. Zhao and J. Yao, "The study of NAO robot arm based on direct kinematics by using D-H method," 2014 UKACC International Conference on Control (CONTROL), Loughborough, 2014, pp. 515-518.

[116] M. A. Ayob, W. N. W. Zakaria and J. Jalani, "Forward kinematics analysis of a 5-axis RV2AJ robot manipulator," 2014 Electrical Power, Electronics, Communicatons, Control and Informatics Seminar (EECCIS), Malang, 2014, pp. 87-92.

[117] Hee-Byoung Choi, A. Konno and M. Uchiyama, "Closed-form solutions for the forward kinematics of a 4-DOFs parallel robot H4," Proceedings 2003 IEEE/RSJ International Conference on Intelligent Robots and Systems (IROS 2003) (Cat. No.03CH37453), Las Vegas, NV, USA, 2003, pp. 3312-3317 vol.3.

[118] H. Wang et al., "Research on the Relationship between Classic Denavit-Hartenberg and Modified Denavit-Hartenberg," 2014 Seventh International Symposium on Computational Intelligence and Design, Hangzhou, 2014, pp. 26-29.

[119] S. Kucuk and Z. Bingul, "Robot Kinematics: Forward and Inverse Kinematics," Industrial Robotics: Theory, Modelling and Control, Sam Cubero, ISBN: 3-86611-285-8, 2006.

[120] L. Sciavicco and B. Siciliano, "Modeling and Control of Robot Manipulators: $2^{\text {nd }}$," Springer publishing 2000, ISBN 1-85233-221-1.

[121] V. D. Tourassis and M. H. Ang, "A modular architecture for inverse robot kinematics," in IEEE Transactions on Robotics and Automation, vol. 5, no. 5, pp. 555-568, Oct. 1989. 
[122] S. Kucuk and Z. Bingul, "The inverse kinematics solutions of industrial robot manipulators," Proceedings of the IEEE International Conference on Mechatronics, 2004. ICM '04., Istanbul, Turkey, 2004, pp. 274-279.

[123] G. Huang, C. Tung, H. Lin and S. Hsiao, "Inverse kinematics analysis trajectory planning for a robot arm," 2011 8th Asian Control Conference (ASCC), Kaohsiung, 2011, pp. 965-970.

[124] C. s. g. Lee and M. Ziegler, "Geometric Approach in Solving Inverse Kinematics of PUMA Robots," in IEEE Transactions on Aerospace and Electronic Systems, vol. AES-20, no. 6, pp. 695706, Nov. 1984.

[125] D. Sandaruwan, N. Kodikara, C. Keppitiyagama and R. Rosa, “A Six Degrees of Freedom Ship Simulation System for Maritime Education," The International Journal on Advances in ICT for Emerging Regions 03, December 2010.

[126] P. Mathys, J. D. Rouck, L. Fernandez, J. Monballiu, D. V. D. Eynde, R. Delgado and A. Dujardin, "Belgian Ocean Energy Assessment (Boreas)," Science for a Sustainable Development, January 2011.

[127] H. Seraji, B. Bon and R. Steele, "Real-time collision avoidance for 7-DOF arms," Proceedings of the 1997 IEEE/RSJ International Conference on Intelligent Robot and Systems. Innovative Robotics for Real-World Applications. IROS '97, Grenoble, France, 1997, pp. 16941699 vol.3.

[128] A. Jacoff, K. Saidi, R. Von Loewenfeldt and Y. Koibuchi, "Development of standard test methods for evaluation of ROV/AUV performance for emergency response applications," OCEANS 2015 - MTS/IEEE Washington, Washington, DC, 2015, pp. 1-10.

[129] A. Jacoff, E. Messina, B. Weiss, S. Tadakoro and Y. Nakagava, "Test Arenas and Performance Metrics for Urban Search and Rescue Robots," IEEE/RSJ International Conference on Intelligent Robots and systems, pp.3396-3403, 2003.

[130] A. Jacoff, E. Messina and B. Weiss, "Intelligent Systems for Urban Search and Rescue: Challenges and Lessons Learned," Proceedings of SPIE - The International Society for Optical Engineering, 2003, vol.5071, doi: 10.1117/12.502415.

[131] A. Jacoff et al., "Events for the application of measurement science to evaluate ground, aerial, and aquatic robots," 2017 IEEE International Symposium on Safety, Security and Rescue Robotics (SSRR), Shanghai, 2017, pp. 131-132.

[132] A. Jacoff, H. Huang, A. Virts, A. Downs and R. Sheh, "Emergency Response Robot Evaluation Exercise," 2012. 
[133] R. Sheh, B. Collidge, M. Lazarescu, H. Komsuoglu and A. Jacoff, "The Response Robotics Summer School 2013: Bringing responders and researchers together to advance Response Robotics,” 2014 IEEE/RSJ International Conference on Intelligent Robots and Systems, Chicago, IL, 2014, pp. 1862-1867.

[134] S. Tadokoro and A. Jacoff, "Performance Metrics for Response Robots [Industrial Activities]," in IEEE Robotics \& Automation Magazine, vol. 18, no. 3, pp. 12-14, Sept. 2011.

[135] F. Maric, I. Jurin, I. Markovic, Z. Kalafatic and I. Petrovic, "Robot arm teleoperation via RGBD sensor palm tracking," $201639^{\text {th }}$ International Convention on Information and Communication Technology, Electronics and Microelectronics Security \& Rescue Robotics (SSRR 2009), Denver, CO, 2009, pp. 1-6.

[136] L. V. Herlant, R. M. Holladay and S. S. Srinivasa, "Assistive teleoperation of robot arms via automatic time-optimal mode switching," $201611^{\text {th }}$ ACM/IEEE International Conference on Human-Robot Interaction (HRI), Christchurch, 2016, pp. 35-42.

[137] G. S. Gupta, S. C. Mukhopadhyay, C. H. Messom and S. Demidenko, "Master-Slave Control of a Teleoperated Anthropomorphic Robotic Arm with Gripping Force Sensing," 2005 IEEE Instrumentation and Measurement Technology Conference Proceedings, Ottawa, Ont., 2005, pp. 2203-2208.

[138] J. Kim, Y. Choi and J. Lee, "Development of a master station for the remote control of rescue robots," $201212^{\text {th }}$ International Conference on Control, Automation and Systems, JeJu Island, 2012, pp. 1994-1997.

[139] A. Franchi, C. Secchi, H. I. Son, H. H. Bulthoff and P. R. Giordano, "Bilateral Teleoperation of Groups of Mobile Robots With Time-Varying Topology," in IEEE Transactions on Robotics, vol. 28, no. 5, pp. 1019-1033, Oct. 2012.

[140] J. Kofman, X. Wu, T. J. Luu and S. Verma, "Teleoperation of a robot manipulator using a vision-based human-robot interface," in IEEE Transactions on Industrial Electronics, vol. 52, no. 5, pp. 1206-1219, Oct. 2005.

[141] A. Birk, S. Schwertfeger, K. Pathak and N. Vaskevicius, "3D data collection at Disaster City at the 2008 NIST Response Robot Evaluation Exercise (RREE)," 2009 IEEE International Workshop on Safety, Security \& Rescue Robotics (SSRR 2009), Denver, CO, 2009, pp. 1-6.

[142] K. Chen et al., "A preliminary study on a groping framework without external sensors to recognize near-environmental situation for risk-tolerance disaster response robots," 2017 IEEE 
International Symposium on Safety, Security \& Rescue Robotics (SSRR), Shanghai, 2017, pp. 181186.

[143] J. Tran, A. Ufkes, M. Fiala and A. Ferworn, "Low-cost 3D scene reconstruction for response robots in real-time," 2011 IEEE International Symposium on Safety, Security \& Rescue Robotics, Kyoto, 2011, pp. 161-166.

[144] Kinova Robotics , "MICO ${ }^{2}$ User Guide," [Online]. Available: https://beta.kinovarobotics.com/sites/default/files/KINOVA_MICO_Robotic_arm_USER_GUID E.pdf. [Accessed Jun. 25, 2018]

[145] Pocket Guide, "RESPONSE ROBOTS DHS/NIST Sponsored Evaluation Exercises," [Online]. Available: https://www.nist.gov/system/files/documents/el/isd/ms/2010_RobotGuide2.pdf. [Accessed Mar. 15, 2019]

[146] ASTM WK54276, "New Test Method for Evaluating Ground Response Robot Dexterity: Grasp Load, Traverse, and Place," [Online].

Available: https://www.astm.org/DATABASE.CART/WORKITEMS/WK54276.htm. [Accessed Mar. 18, 2019] 